Florida International University FIU Digital Commons

7-5-2017

\title{
Band Gap Engineering of 2D Nanomaterials and Graphene Based Heterostructure Devices
}

MD Monirojjaman MONSHI

Florida International University, mmons021@fiu.edu

DOI: $10.25148 /$ etd.FIDC001977

Follow this and additional works at: https://digitalcommons.fiu.edu/etd

Part of the Electrical and Computer Engineering Commons

\section{Recommended Citation}

MONSHI, MD Monirojjaman, "Band Gap Engineering of 2D Nanomaterials and Graphene Based Heterostructure Devices" (2017). FIU Electronic Theses and Dissertations. 3354.

https://digitalcommons.fiu.edu/etd/3354 


\section{FLORIDA INTERNATIONAL UNIVERSITY}

Miami, Florida

\section{BAND GAP ENGINEERING OF 2D NANOMATERIALS AND GRAPHENE BASED HETEROSTRUCTURE DEVICES}

A dissertation submitted in partial fulfillment of

the requirements for the degree of

DOCTOR OF PHILOSOPHY

in

ELECTRICAL ENGINEERING

by

Md Monirojjaman Monshi 
To: Interim Dean Ranu Jung

College of Engineering and Computing

This dissertation, written by Md Monirojjaman Monshi and entitled Band Gap Engineering of 2D Nanomaterials and Graphene Based Heterostructure Devices, having been approved in respect to style and intellectual content, is referred to you for judgment.

We have read this dissertation and recommend that it be approved.

Sakhrat Khizroev

Nezih Pala

Peggy Chunlei Wang

Iftekhar Chowdhury

Shekhar Bhansali, Co-Major Professor

Irene Calizo, Co-Major Professor

Date of Defense: July 5, 2017

The dissertation of Md Monirojjaman Monshi is approved.

Interim Dean Ranu Jung

College of Engineering and Computing

Andrés G. Gil

Vice President for Research and Economic Development and Dean of the University Graduate School

Florida International University, 2017 
C Copyright 2017 by Md Monirojjaman Monshi

All rights reserved. 


\section{DEDICATION}

I dedicate this dissertation to my parents, brothers, loving wife, and in-laws. Without their love, support, and patience, it would have been impossible to complete this research. 


\section{ACKNOWLEDGMENTS}

First and foremost, I would like to express gratitude to my advisor and mentor, Dr. Irene Calizo, for giving me the opportunity to work on an exciting project for my doctoral dissertation. I am also thankful for his encouragement and support in developing my research skills and personality through the many opportunities provided during my time at Florida International University.

I would like to thank the members of my dissertation committee Dr. Sakhrat Khizroev, Dr. Nezih Pala, Dr. Peggy Chunlei Wang, Dr. Ifterkhar Chowdhury and Co-Major Professor Dr. Shekhar Bhansali, for agreeing to be my dissertation committee, and for their valuable inputs towards my dissertation research.

I take this opportunity to also thank all the members of the QUEST Lab and other graduate students that worked along with me at Electrical and Computer Engineering and Advanced Materials Engineering Research Lab. Thanks to Patrick Roman, Neal Ricks, and Dr. Alexander Franco to help me out in the clean room. Many thanks to my dearest friends who have supported me and stood by me through the hardest times of this journey, Sadegh Aghaei and Ingrid Torres.

I would like to acknowledge the funding sources that made this work possible: Graduate assistantship from the Department of Electrical and Computer Engineering at Florida International University.

Finally, I could not have come this far without the unconditional love, support, patience and blessings from my family. Many thanks to my wife Nasrin Sultana and my in-laws. 


\title{
ABSTRACT OF THE DISSERTATION \\ BAND GAP ENGINEERING OF 2D NANOMATERIALS AND GRAPHENE \\ BASED HETEROSTRUCTURE DEVICES \\ by
}

Md Monirojjaman Monshi

Florida International University, 2017

\author{
Miami, Florida \\ Professor Irene Calizo, Co-Major Professor \\ Professor Shekhar Bhansali, Co-Major Professor
}

Two-Dimensional (2D) materials often exhibit distinguished properties as compared to their $3 \mathrm{D}$ counterparts and offer great potential to advance technology. However, even graphene, the first synthesized 2D material, still faces several challenges, despite its high mobility and high thermal conductivity. Similarly, germanene and silicene face challenges due to readily available semiconducting properties to be used in electronics, photonics or photocatalysis applications. Here, we propose two approaches to tune the band gap: One is by forming nanoribbon and edge functionalization and another by doping using inorganic nanoparticle's interaction with 2D nanomaterials.

Edge functionalization of armchair germanene nanoribbons (AGeNRs) has the potential to achieve a range of band gaps. The edge atoms of AGeNRs are passivated with hydrogen ($\mathrm{H}$ and $-2 \mathrm{H})$ or halogen $(-\mathrm{F},-\mathrm{Cl},-\mathrm{OH},-2 \mathrm{~F},-2 \mathrm{Cl})$ atoms. Using density functional theory calculations, we found that edge-functionalized AGeNRs had band gaps as small as 0.012 $\mathrm{eV}$ when functionalized by $-2 \mathrm{H}$ and as high as $0.84 \mathrm{eV}$ with $-2 \mathrm{~F}$. 
Doping can change the semiconducting behavior of AGeNRs to metal due to the half-filled band making it useful for negative differential resistance (NDR) devices. In the case of zigzag germanene nanoribbons (ZGeNRs), single $\mathrm{N}$ or B doping transformed them from anti-ferromagnetic (AFM) semiconducting to ferromagnetic (FM) semiconductor or halfmetal. Lastly, formation and edge free energy studies revealed the feasibility of chemical synthetization of edge-functionalized and doped germanene.

Electronic, optical and transport properties of the graphene/ $\mathrm{ZnO}$ heterostructure have been explored using first-principles density functional theory. The results show that $\mathrm{Zn}_{12} \mathrm{O}_{12}$ can open a band gap of $14.5 \mathrm{meV}$ in graphene, increase its optical absorption by 1.67 times, covering the visible spectrum and extended to the infra-red (IR) range, and create slight nonlinear $I-V$ characteristics depending on the applied bias. This agrees well with collaborative experimental measurement of a similar system.

In conclusion, we have successfully studied the potential use of edge functionalization, band gap periodicity in nanoribbon width, and doping in germanene nanoribbons. Structural stability was also studied to investigate the feasibility for experimental synthesization. Inorganic nanoparticle's interaction with graphene envisages the possibility of fabricating photo-electronic device covering visible spectrum and beyond. Finally, graphene complexes were merged with naturally available direct band gap of monolayer $\mathrm{MoS}_{2}$ to build efficient energy harvesting and photo detecting devices. 


\section{TABLE OF CONTENTS}

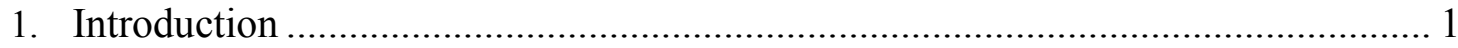

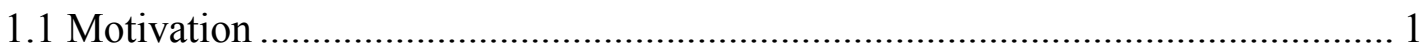

1.2 Research Objectives and Challenges .......................................................... 3

1.2.1 Objectives 3

1.2.2 Challenges 4

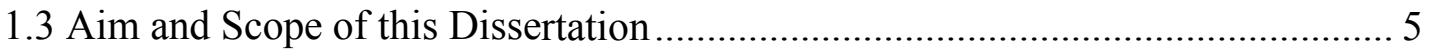

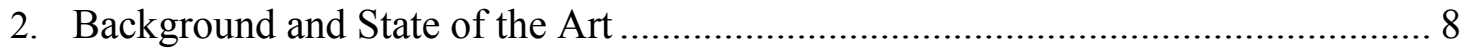

$2.12 \mathrm{D}$ materials overview: graphene and beyond ............................................... 8

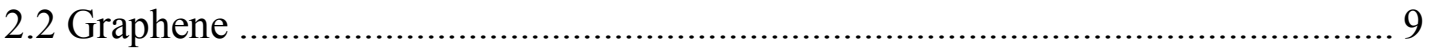

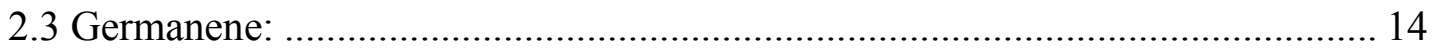

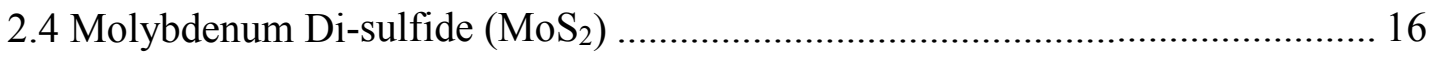

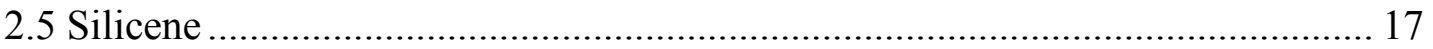

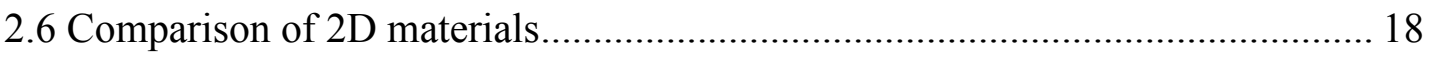

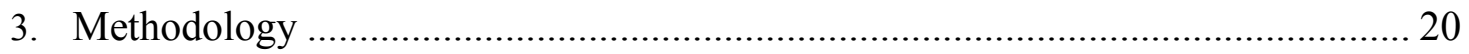

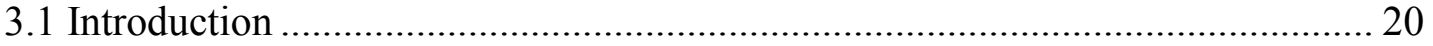

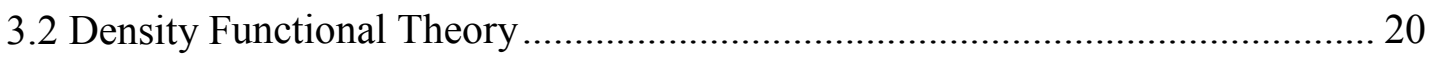

3.2.1 Born-Oppenheimer Approximation 21

3.2.2 Hohenberg-Kohn Theorems 21

3.2.3 Kohn-Sham Equations $\quad 22$

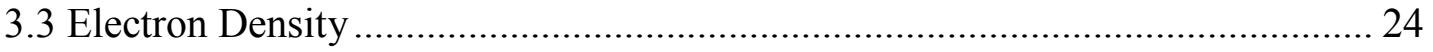

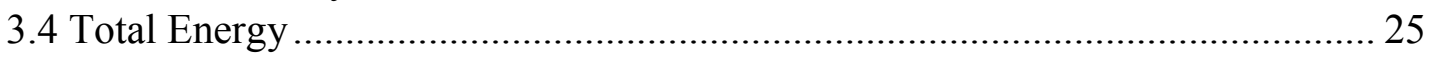

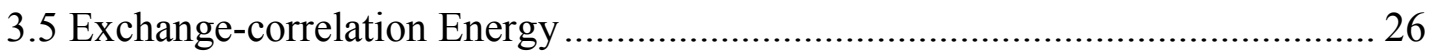

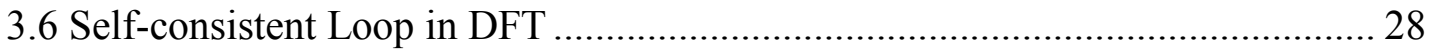

3.7 van der Waals Contribution in DFT .............................................................. 29

3.7.1 D2 correction 30

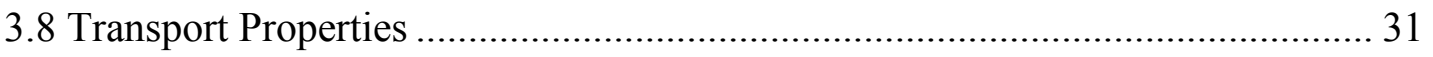

4. Edge-functionalized germanene nanoribbons: impact on electronic and magnetic

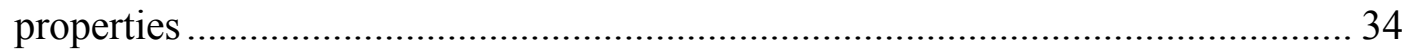

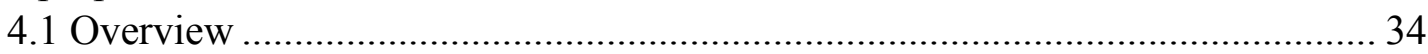

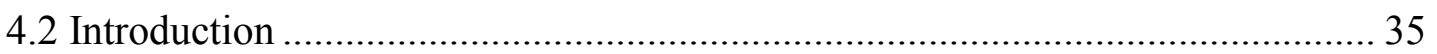

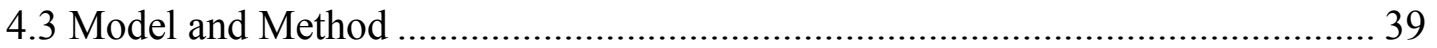

4.5 Armchair Germanene Nanoribbon .............................................................. 40

4.5.1 Stability of AGeNRs. $\quad 40$

4.5.2 Electronic properties of AGeNRs $\quad 44$

4.6.1 Stability of ZGeNRs 48 
4.6.2 Electronic and magnetic properties of ZGeNRs 50

4.7 Armchair germanene nanoribbons doped by $\mathrm{N}$ or B atoms ............................ 51

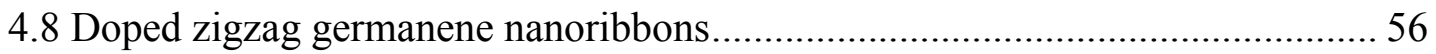

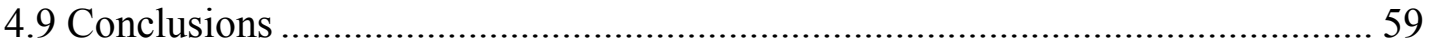

5. Doping and Defect Induced Germanene: A Superior Media for Sensing $\mathrm{H}_{2} \mathrm{~S}, \mathrm{SO}_{2}$,

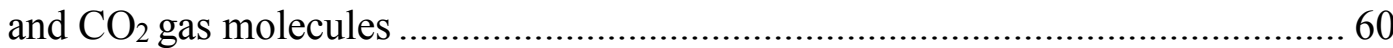

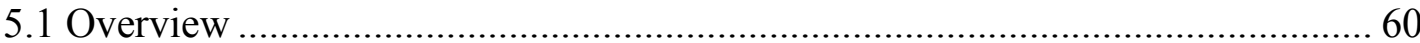

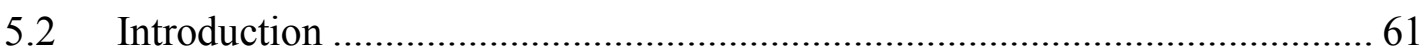

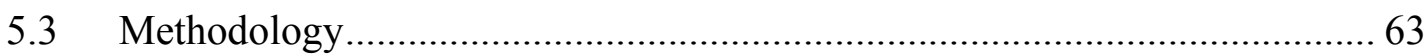

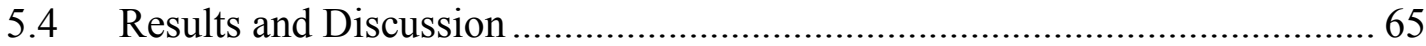

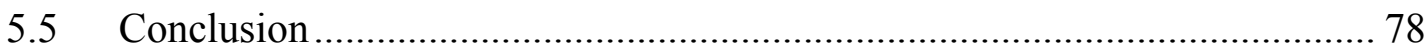

6. Band Gap Opening and Optical Absorption Enhancement in Graphene using ZnO

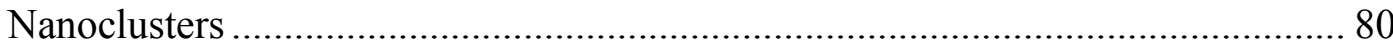

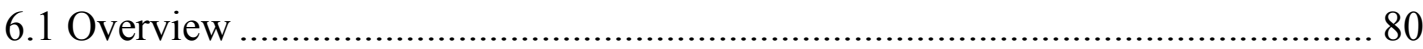

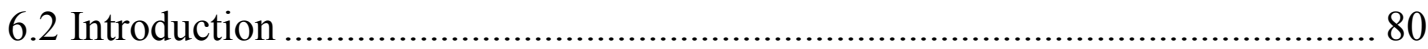

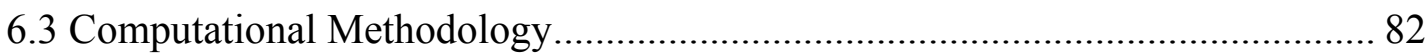

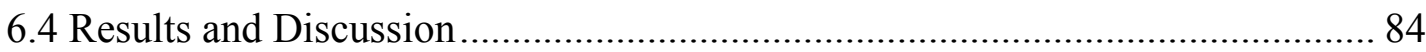

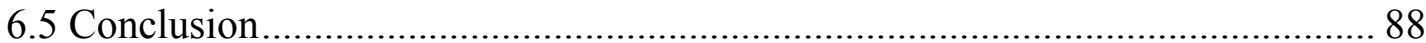

7. $\mathrm{ZnO}$ nanocluster enhanced optical and electronic properties graphene/MoS2 ......89

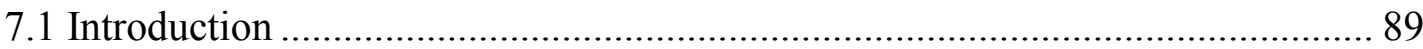

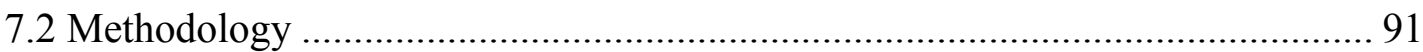

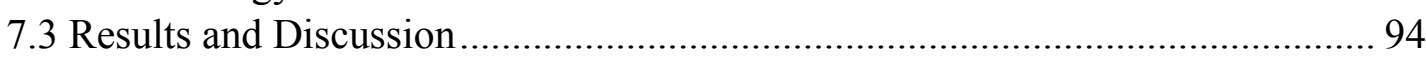

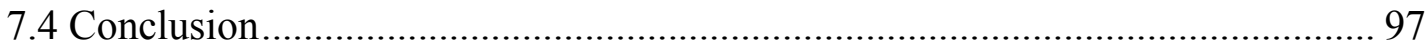

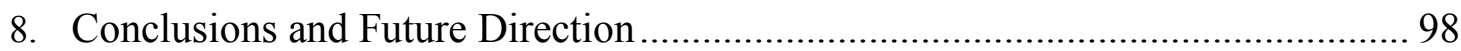

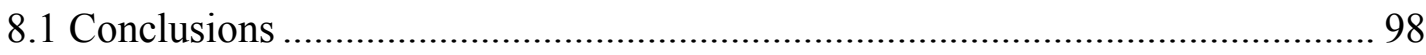

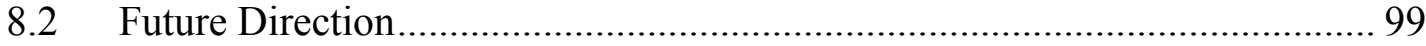

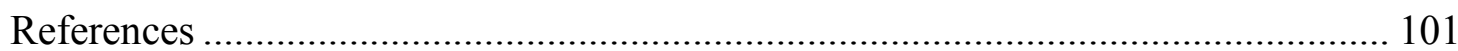

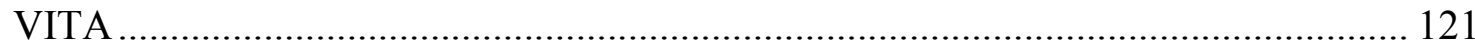




\section{LIST OF FIGURES}

FIGURE

PAGE

Figure 2.1 Electronic States of Carbon...................................................................... 10

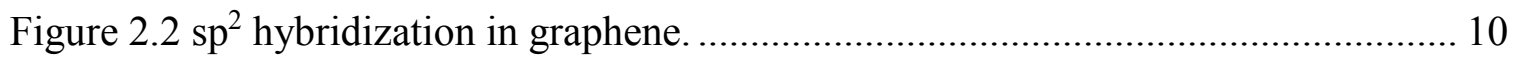

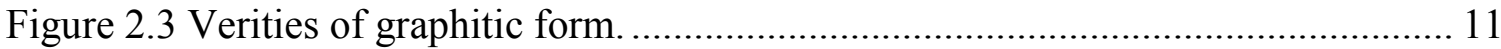

Figure 2.4 (a) Conventional Semiconductors and their electrons exhibit a parabolic relationship between their energy (E) and momentum (p), (b) Two-dimensional (2D) Dirac fermions, such as electrons in graphene, have valence (blue) and conduction (purple) energy bands with a linear energy-momentum relationship. These touch at a point called the Dirac point in the 3D parameter space formed by E, $\mathrm{p}_{\mathrm{x}}$, and $\mathrm{p}_{\mathrm{y}}$. Shown here is a $2 \mathrm{D}$ slice of the $3 \mathrm{D}$ space, (c) A 3D slice of the $4 \mathrm{D}\left(\mathrm{E}, \mathrm{p}_{\mathrm{x}}, \mathrm{p}_{\mathrm{y}}, \mathrm{p}_{\mathrm{z}}\right)$ energymomentum relationship of 3D Dirac fermions such as those discovered [78-81] in $\mathrm{Cd}_{3} \mathrm{As}_{2}$, with two Dirac points along a special high-symmetry axis $\left(\mathrm{p}_{\mathrm{z}}\right)$.

Figure 2.5 (a) The top and side views of the low-buckled germanene sheet, the hexagonal lattice constant, a, and the buckling height, b, are indicated, (b) The band structure of low-buckled germanene with a Fermi level set at zero energy indicated by the red dashed line.

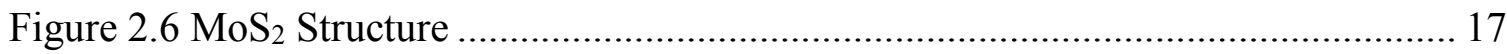

Figure 2.7 Band structure and atomic structure of (a) graphene, (b) germanene, (c) $\mathrm{MoS}_{2}$ and (d) hexagonal boron nitride (h-BN).

Figure 3.1 A potential and its corresponding wavefunction of all-electron (blue) compared to that of pseudoelectron (red). Here, $r_{c}$ is the radius at which all-electron and pseudopotential values match [111].

Figure 3.2 A flow chart of the iteration scheme in DFT method for calculating electron density and total energy. 
Figure 3.3 Geometry of a device configuration with two electrodes. The system is periodic in the left and right electrodes. The central region is seamlessly connected to the electrodes. The parts of the central region where the atoms have the arrangement same as electrodes are called the left and right electrode extension.

Figure 4.1 Graphical models of edge-functionalized 7-AGeNRs. L is the periodic length of the structure. The primitive supercell is marked by a dashed box. The cyan and red balls represent germanium and functional addends $(\mathrm{H}, \mathrm{F}, \mathrm{Cl}, \mathrm{Br}$, and $\mathrm{I})$, respectively. $\mathrm{P}_{1}$ and $\mathrm{P}_{2}$ are the preferred position of doping with boron $(\mathrm{B})$ or nitrogen $(\mathrm{N})$.

Figure 4.2 Edge free energy $\gamma_{\mathrm{H} 2}$ of hydrogenated GeNR edge structures versus hydrogen chemical potential $\mu_{\mathrm{H} 2}$, using $\mathrm{E}_{\mathrm{H} 2}$ as the zero reference. The allowed range of hydrogen chemical potential is indicated by vertical solid lines. The bottom inset axis represents pressure, in bar, of the molecular $\mathrm{H}_{2}$ corresponding to $\mathrm{T}=300 \mathrm{~K}$.

Figure 4.3 (a) Band gap variation of AGeNR with mono- and di-hydrogen edge functionalization and (b) effective mass variation with ribbon width for mono and dihydrogen edge functionalization of AGeNRs. Band Structure of (c) 9, (d) 10, (e) 11 and (f) 12 atoms wide hydrogen functionalized armchair germanene nanoribbon. The Fermi level $\left(\varepsilon_{\mathrm{f}}\right)$ is set at zero

Figure 4.4 Ribbon width vs band gap (eV) for (a) mono-edge and (b) di-edge functionalization. Effective mass variation of electron with ribbon width for (c) monoand (d) di- edge functionalization of AGeNRs.

Figure 4.5 Structural configurations of edge-functionalized, along with periodic length L, GeNRs studied. The primitive supercell is marked by a dashed black box. The cyan and red balls represent $\mathrm{Ge}$ and functional addends $(\mathrm{H}, \mathrm{F}, \mathrm{Cl}, \mathrm{Br})$, respectively. $\mathrm{P}_{1}$ and $\mathrm{P}_{2}$ are the preferred position of doping with boron $(\mathrm{B})$ or nitrogen $(\mathrm{N})$.

Figure 4.6 Edge free energy $\gamma_{\mathrm{H} 2}$ of different hydrogenated ZGeNRs edge structures versus hydrogen chemical potential $\mu_{\mathrm{H} 2}$, using $\mathrm{E}_{\mathrm{H} 2}$ as the zero reference. The allowed range of hydrogen chemical potential is indicated by vertical solid lines. The bottom inset axis represents pressure, in bar, of the molecular $\mathrm{H}_{2}$ corresponding to $\mathrm{T}=300 \mathrm{~K} \ldots 49$

Figure 4.7 Band structure of hydrogenated $Z_{11}$ in the (a) NM and (b) AFM states. $\alpha$ (black solid line) and $\beta$ (red dashed line) represent spin-up and spin-down bands, respectively. 
Figure 4.8 Band structure of B-doped fluorinated a11 in the (a) NM and (b) FM states, N-doped fluorinated a11 in the (c) NM and (d) FM states.

Figure 4.9 Band Structure of N-doped hydrogenated $\mathrm{a}_{11}$ in the (a) NM and (b) AFM states, N-doped fluorinated (c) NM and (d) FM states.

Figure 4.10 Effect of doping concentration: band structure of hydrogenated $\mathrm{a}_{11}$ when doped by (a) B, (b) 2B and (c) $3 \mathrm{~B}$

Figure 4.11 Band structure of B-doped hydrogenated $Z_{11}$ in the (a) NM and (b) AFM states and N-doped hydrogenated (c) NM and (d) AFM states.

Figure 4.12 Band structure of B-doped hydrogenated $z_{22}$ in the (a) NM and (b) FM states, B-doped fluorinated (c) NM and (d) AFM states.

Figure 5.1 Top and side view of optimized structures of (a) pristine, (b) mono vacancy, (c) di vacancy, and (d) tri vacancy of germanene sheet. Possible sites for gas molecules adsorption on germanene are: T (top), V (Valley), B (bridge), and H (hollow).

Figure 5.2 Top and side views of optimized structures of pure germanene with (a) $\mathrm{H}_{2} \mathrm{~S}$, (b) $\mathrm{SO}_{2}$, and (c) $\mathrm{CO}_{2}$, monovacancy germanene with (d) $\mathrm{H}_{2} \mathrm{~S}$, (e) $\mathrm{SO}_{2}$, and (f) $\mathrm{CO}_{2}$, divacancy germanene with (g) $\mathrm{H}_{2} \mathrm{~S}$, (h) $\mathrm{SO}_{2}$, and (i) $\mathrm{CO}_{2}$, and trivacancy germanene with (d) $\mathrm{H}_{2} \mathrm{~S}$, (e) $\mathrm{SO}_{2}$, and (f) $\mathrm{CO}_{2}$, respectively. Cyan, green, yellow, red and brown balls represent $\mathrm{Ge}, \mathrm{H}, \mathrm{S}, \mathrm{O}$, and $\mathrm{H}$ atoms, respectively.

Figure 5.3 van der Waals induced adsorption energies of incident gases on pristine, defected, and substituted germanene.

Figure 5.4 The density of states (DOS) of the pristine germanene nanosheet adsorbed by (a) $\mathrm{H}_{2} \mathrm{~S}$ and (b) $\mathrm{SO}_{2}$ and (c) $\mathrm{CO}_{2}$ molecule. The grey shaded area represents the total DOS, while the red, blue, and green colors account for the projection of DOS onto $\mathrm{H}_{2} \mathrm{~S}$, $\mathrm{SO}_{2}$, and $\mathrm{CO}_{2}$ molecule, respectively. Here the Fermi level is shifted to zero......

Figure 5.5 The density of states (DOS) of the vacancy defected germanene nanosheet adsorbed by (a) $\mathrm{H}_{2} \mathrm{~S}$ and (b) $\mathrm{SO}_{2}$ and (c) $\mathrm{CO}_{2}$ molecule. The grey shaded area represents the total DOS, while the red, blue, and green colors account for the projection of DOS onto $\mathrm{H}_{2} \mathrm{~S}, \mathrm{SO}_{2}$, and $\mathrm{CO}_{2}$ molecule, respectively. Here the Fermi level is shifted to zero. 76 
Figure 6.1 (a) Optimized $\mathrm{Zn}_{12} \mathrm{O}_{12}$ nanocluster and (b) orbital energy levels diagram of

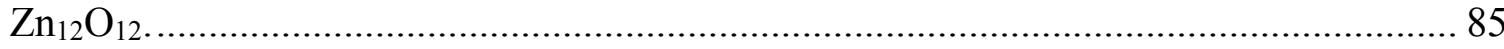

Figure 6.2 Graphene/ $\mathrm{Zn}_{12} \mathrm{O}_{12}$ atomic structure, (a) top view, (b) side view, where gray as carbon, cyan as zinc and red ball as oxygen, (c) two electrode system of Graphene/

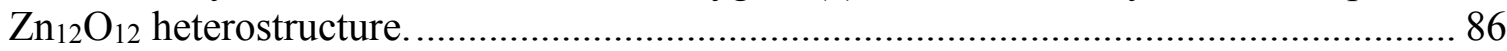

Figure 6.3 (a) Bandstructure and (b) Density of states of Graphene/ $\mathrm{Zn}_{12} \mathrm{O}_{12}$.

Figure 6.4 (a) optical absorption coefficient and (b) I-V characteristics of graphene/ $\mathrm{Zn}_{12} \mathrm{O}_{12}$ two electrode system

Figure 7.1 $\mathrm{Zn}_{12} \mathrm{O}_{12} /$ Graphene/ $\mathrm{MoS}_{2}$ atomic structure, (a) top view, (b) side view, where gray as carbon, cyan as zinc and red ball as oxygen, (c) two electrode system of $\mathrm{Zn}_{12} \mathrm{O}_{12} /$ Graphene/ $\mathrm{MoS}_{2}$ heterostructure.

Figure 7.2 (a) Bandstructure and (b) Density of states of $\mathrm{Zn}_{12} \mathrm{O}_{12} /$ Graphene/ $\mathrm{MoS}_{2} \ldots \ldots . . .95$

Figure 7.3 (a) optical absorption coefficient and (b) I-V characteristics of $\mathrm{Zn}_{12} \mathrm{O}_{12} /$ Graphene/ $\mathrm{MoS}_{2}$ two electrode system. 


\section{LIST OF ACRONYMS \& ABBREVIATIONS}

\begin{tabular}{|c|c|}
\hline FET & Field-effect transistor \\
\hline $3 \mathrm{D}$ & Three-dimensional \\
\hline $2 \mathrm{D}$ & Two-dimensional \\
\hline $1 \mathrm{D}$ & One-dimensional \\
\hline $0 \mathrm{D}$ & Zero-dimensional \\
\hline SiNR & Silicene nanoribbon \\
\hline AGeNR & Armchair germanene nanoribbon \\
\hline ZGeNR & Zigzag germanene nanoribbon \\
\hline GNR & Graphene nanoribbon \\
\hline AGNR & Armchair graphene nanoribbon \\
\hline GO & Graphene oxide \\
\hline CNT & Carbon nanotube \\
\hline SOC & Spin-orbit coupling \\
\hline NM & Nonmagnetic \\
\hline FM & Ferromagnetic \\
\hline AFM & Antiferromagnetic \\
\hline $\mathrm{vdW}$ & van der Waals \\
\hline $\mathrm{BZ}$ & Brillouin zone \\
\hline PDOS & Partial density of states \\
\hline CBM & Conduction band minimum \\
\hline
\end{tabular}




\begin{tabular}{|c|c|}
\hline VBM & Valence band maximum \\
\hline DFT & Density functional theory \\
\hline NEGF & Non-equilibrium Green's function \\
\hline LDA & Local density approximation \\
\hline PZ & Perdew-Zunger \\
\hline PW & Perdew-Wang \\
\hline VWN & Vosko-Wilk-Nusair \\
\hline GGA & Generalized gradient approximation \\
\hline PW91 & Perdew-Wang 1991 \\
\hline $\mathrm{PBE}$ & Perdew-Burke-Ernzerhof \\
\hline B3LYP & Becke, 3-parameter, Lee-Yang-Parr \\
\hline TPSS & Tao-Perdew-Staroverov-Scuseria \\
\hline HSE06 & Heyd-Scuseria-Ernzerhof 06 \\
\hline BSSE & Basis set superposition errors \\
\hline LCAP & Linear combination of atomic orbitals \\
\hline $\mathrm{cp}$ & Counterpoise \\
\hline ATK & ATOMISTIX TOOLKIT \\
\hline TEM & Transmission electron microscopy \\
\hline STM & Scanning tunneling microscopy \\
\hline AFM & Atomic force microscopy \\
\hline RHEPD & reflection high-energy positron diffraction \\
\hline AREPS & angle-resolved photoelectron emission spectroscopy \\
\hline
\end{tabular}


LEED

UHV

CVD

UV
Low energy electron diffraction

Ultra-high vacuum

Chemical vapor deposition

Ultraviolet 


\section{CHAPTER 1}

\section{Introduction}

\subsection{Motivation}

The so-called famous scotch-tape exfoliation and characterizations of graphene in 2004, by Novoselov and Geim et al., has started a new quest towards two dimensional (2D) materials for solid-state science and technologies [1]. Graphene, a monolayer of $\mathrm{sp}^{2}$ carbon, exhibits distinguishing properties that no other material can reach, such as exceptionally high electron mobility $\left(-200,000 \mathrm{~cm}^{2} \mathrm{~V}^{-1} \mathrm{~s}^{-1}\right)$ [2], remarkably large thermal conductivity $(\sim 4.84 \pm 0.44) \times 10^{3}$ to $\left.5.30 \pm 0.48 \times 10^{3} \mathrm{~W} / \mathrm{mK}\right)$ [3], excellent optical conductance [4], high stiffness (1 Tpa) [5] and anomalous quantum Hall effect [6]. This effort is followed by further explorations of other 2D materials, such as transition metal dichalcogenides (TMDCs) [7], puckered silicene [8-10], germanene [11] and phosphorene [12]. Among them, graphene, germanene and silicene do not have intrinsic band gap and behave like massless Dirac fermions [6, 13-16].

Nonetheless, large-scale production of graphene for practical applications faces two major challenges. Firstly, one is the synthesis of high-quality graphene in industrial scale. Secondly, engineering the 2D structures with a suitable band gap for electronic and photonic devices. For the first challenge, many approaches have been undertaken, such as patterned epitaxial growth on silicon carbide substrate [17, 18]. A relatively low-cost approach is liquid-phase exfoliation of graphite through the use of graphene intercalated compounds $[19,20]$. The most common method of generating a large area of graphene 
with no or low defect densities is to use chemical vapor deposition (CVD) on a metal substrate, mostly copper [21, 22] and nickel substrate [23-25]. Free standing germanene and silicene are unstable in the air and get oxidized easily [26]. Graphene-like germanene sheets have been produced on $\operatorname{Pt}(111), \mathrm{Ge} / \mathrm{Ag}(111)$, and $\mathrm{Al}(111)$ substrates and were also epitaxially and mechanically exfoliated using $\mathrm{GeH}$ synthesis on $\mathrm{SiO}_{2}$ [27-30]. Since the prediction by Takeda et al. different monolayer silicene superstructures have been successfully synthesized on various substrates, including $\mathrm{Ag}(111), \operatorname{Ir}(111), \mathrm{ZrB}_{2}(0001)$, $\operatorname{ZrC}(111)$ and $\mathrm{MoS}_{2}$ surfaces $[8,15,16,31,32]$. Multilayer silicene sheets have also been grown on $\mathrm{Ag}(111)$ surface [10]. Large scale production of monolayer and multilayer $\mathrm{MoS}_{2}$ includes shear exfoliation [33], liquid phase exfoliation [34], low-energy ball milling and sonication [35].

For the second challenge, the unavailability of a bandgap makes graphene challenging to integrate into high-performance logic circuits [36]. In order to "engineer" the band gap, many attempts are devoted to nanostructuring of graphene into nanoribbons, [37] and bilayers [38, 39], chemical modifications [40] and tuning by a substrate [41]. So far, no desirable achievement has been reached for graphene. Furthermore, once graphene is maintained on a substrate, substrate-induced structural distortion [42], local charge disorder [43], edge effects [44], defects and impurities [45] would strongly affect its properties. Edge functionalization [46, 47], stress [48], doping [49], defects, confinement in the form of nanoribbon [46, 50], nanomesh [51, 52], interaction of inorganic nanoparticles [53] etc. are the possible solutions to open a band gap in 2D nanomaterials. 
Apart from the bandgap issue of graphene, other 2D nanomaterials like $\mathrm{MoS}_{2}$ and black phosphorene has a natural band gap. $\mathrm{MoS}_{2}$ is a transition metal dichalcogenides (TMDC) of particular significance since it possesses an indirect-gap of $1.20 \mathrm{eV}$ but when reduced to a monolayer, it changes into direct bandgap material with a bandgap of $1.80 \mathrm{eV}$ due to quantum confinement [54-56]. A direct band gap in black phosphorous scales as the inverse number of layers and changes significantly between $1.0 \mathrm{eV}$ in a single layer and $0.3 \mathrm{eV}$ in the bulk [57].

To make use of the high carrier mobility of graphene in devices, 2D transition metal dichalcogenides (TMDCs) such as $\mathrm{MoS}_{2}$ with a direct band gap $(1.8 \mathrm{eV})$ can be stacked together to build van der Waals (vdW) heterostructures. In fact, several experimental works suggest that new or tailored properties could be achieved by assembling graphene with various 2D layers into artificial heterostructures [58-61]. Graphene and MoS2, as twodimensional (2D) nanomaterials, hold great promise in the next-generation electronic and photonic applications because of the exceptional properties gained from the ultra-thin planar structures, such as strong electron-hole confinement, extreme bendability, and high transparency, which pave the way for the fabrication of ultra-thin, flexible and efficient devices $[36,55]$.

\subsection{Research Objectives and Challenges}

\subsubsection{Objectives}

The objective of the current research is to delve into the electronic, magnetic and optical properties and photo performance of emerging 2D nanomaterials such as graphene, germanene, $\mathrm{MoS}_{2}$, and graphene-MoS $\mathrm{M}_{2}$ heterostructures through first principles modeling. 
The objectives of the project are: 1) to investigate different approaches to tune 2D nanomaterials bandgap; 2) to understand the fundamental mechanisms of the effects of defects and doping; 3) building heterostructure to improve device electronic and optical performance.

Theoretically, optical absorption and electronic properties of band gap engineered graphene, germanene and $\mathrm{MoS}_{2}$ sheets and nanoribbons using first principle method will be explored. The optical spectrum, dielectric constant, absorption coefficient, and electronic band structures will be studied for graphene, $\mathrm{MoS}_{2}$, and their heterostructures. The effects of doping, defects, inter-layer distance, stacking order and geometry of the heterostructures on photoresponsivity of the photodetectors will also be considered. The thickness-dependent responsivity of $\mathrm{MoS}_{2}$ and graphene thin films, as well as nanoseeds such as $\mathrm{ZnO}$, Au's impact on photodetection, will also be studied using first principles calculations.

\subsubsection{Challenges}

\section{i) Improvement of on/off ratio of the photodetector through enhanced I-V} characteristics: graphene/ $\mathrm{MoS}_{2}$ heterostructure photodetectors should have a very high gain but suffer from low on/off ratio because graphene intrinsically has a low on/off current. To achieve improved on/off switching ratio in the layered structure, graphene nanoribbon or band gapped engineered graphene can be an option. In that case either doping or graphene decorated with nanoseeds or nanopillars of $\mathrm{ZnO}, \mathrm{Au}$ etc. can open a new avenue to improve photodetector's on/off ratio. 
ii) Tunability and Responsivity of Photodetector: Most of the photodetectors reported thus far have been limited by narrow spectral range and long response time. The novel device should be able to respond to a broader range of optical spectrum with high responsivity so that it would be capable of identifying a particular wavelength in a larger spectrum. To address this issue n-type $\mathrm{MoS}_{2}$ stacking with n-type graphene $\left(\mathrm{Zn}_{12} \mathrm{O}_{12}\right.$ nanoclusters induced charge transfer from graphene) can be used to lower the Schottky barrier height in a $\mathrm{Zn}_{12} \mathrm{O}_{12} /$ graphene/ $\mathrm{MoS}_{2}$ heterostructure with a wide spectral range of detection.

\subsection{Aim and Scope of this Dissertation}

The major objective of this dissertation is to investigate different techniques such as doping, defects and edge functionalization to open bandgap in 2D nanomaterials and their influence on electronic, magnetic and optical characteristics. Moreover, quantum techniques such as forming nanoribbon, nanomesh, nanoholes etc. are also investigated to study the impact on band gap tuning and magnetic properties of $2 \mathrm{D}$ materials. Finally, to make use of bandgap engineered materials, different gas sensing and photo-electronic devices are studied. Chapter 2 provides background and state of the art different 2D form of materials such as graphene, germanene, silicene and $\mathrm{MoS}_{2}$. Their synthesis procedures to large scale production and potential techniques for bandgap engineering in those $2 \mathrm{D}$ materials have been discussed in this chapter. Finally, comparative study of those materials also discussed in brief.

Chapter 3 introduces the theoretical toolbox applied in this thesis. We briefly retrace the underlying theory leading to the pragmatic aspects of density- functional theory (DFT). 
The starting point is the quantum mechanical many-body problem, and the BornOppenheimer (BO) approximation, Sec. 3.2.1. The BO approximation formally separates the electronic coordinates from the nuclear ones. The electronic sub-problem is then solved for a fixed set of nuclear coordinates greatly simplifying the many-body problem. Then, the electronic Schrödinger equation is tackled in the DFT framework. The foundation of DFT is the Hohenberg-Kohn theorems Sec. 3.2.2. Using the Hohenberg-Kohn theorems, the electronic many-body Hamiltonian is rewritten into a set of single-particle Schrödinger equations, the Kohn-Sham equations. To finally turn DFT into practice for system sizes up to several thousand atoms, approximations for the unknown terms in the Kohn-Sham equations have to be introduced. For a reliable description of solids, surfaces or weakly bonded layered materials, such as graphite, it is essential to include van-der-Waals (vdW) contributions (Sec. 2.4). Once the Kohn-Sham equations are solved, in this work using the ATOMIZTIX toolkit, the resulting total energy can be used to determine the stability of structures. The Kohn-Sham eigenvalues can be used to obtain a first impression of the electronic structure of the system.

Chapter 4 investigates the edge-functionalized germanene and how to open a band gap in buckled structures of this special materials while breaking the lattice symmetry. Structural stability and experimental conditions have been evaluated in this chapter. Electronic and magnetic properties of doped armchair and zigzag germanene nanoribbon have also been studied in this chapter. Moreover, the impact of doping concentration on electronic and magnetic properties of germanene nanoribbon have been observed. Chapter 5 introduces the impact of doping and vacancy defect in germanene nanosheet for sensing toxic gases 
such as $\mathrm{H}_{2} \mathrm{~S}, \mathrm{SO}_{2}$ and $\mathrm{CO}_{2}$. Chapter 6 delve into the possible techniques to open a band gap in graphene and enhancement of optical properties using $\mathrm{ZnO}$ nanocluster. Chapter 7 details the absorption spectra of $\mathrm{ZnO} /$ graphene/ $\mathrm{MoS}_{2}$ heterostructure and changes in $I-V$ characteristics compared to graphene/MoS 2 heterostructure.

Finally, chapter 8 , we summarize the contribution of this dissertation, future works and proposing future photo-electronic devices. 


\section{CHAPTER 2}

\section{Background and State of the Art}

\subsection{D materials overview: graphene and beyond}

The first semiconducting transistor was made of germanium in 1948, but silicon quickly rose to become the dominant semiconductor material[62]. Silicon is mechanically strong, incredibly abundant: 28.2 percent of the Earth's crust is silicon, relatively easy to purify, and has reasonable electrical properties. This nearly-perfect semiconductor functioned well for making diodes and transistors and still is the basis of almost every computer chip in the market. But, silicon is very inefficient at converting light into an electrical signal or turning the electricity back into the light and hard to miniaturize devices to the nanoscale. When the primary use of semiconductors is in computer processors connected by metal wires, this wasn't much of a problem. But, as we progressed toward using semiconductors in solar panels, camera sensors, and other light-related applications, this weakness of silicon became a real obstacle to progress[63].

The second half of the twentieth century was ruled by the fabrication of microelectronics based on the semiconducting silicon. Silicon undeniably will continue to play a central role in the development and production of new electronic devices. Nowadays, our ambition extended to develop electronic devices at the nanoscale. To meet the tasks that come with advances in building electronic devices as small as a few nanometer demanding new materials to be evaluated. A promising material candidate to lead the arena of device design 
is graphene. Graphene is a two-dimensional atom-thick layer of carbon atoms, ordered in a honeycomb structure. For many years graphene was considered a theoretician's toy model and was assumed not to exist in a free state [64]. Novoselov et al. [1] thrived to isolate a single layer of graphene from a pencil stroke using Scotch tape. Ironically, their low-tech approach paved the way for the production of nanoscale devices [65-71]. Graphene's exceptional electronic and structural properties draw immense attention to the solid-state community [6, 72-74]. In 2010, Andre K. Geim and Konstantin S. Novoselov at the University of Manchester were awarded the Nobel Prize in Physics "for groundbreaking experiments regarding the two-dimensional material graphene".

\subsection{Graphene}

Carbon, the elementary constituent of graphene and graphite, is the $6^{\text {th }}$ element of the periodic table. Its atom is, therefore, built from 6 protons, 6 neutrons, and 6 electrons. In the atomic ground state, the 6 electrons are in the configuration $1 s^{2} 2 s^{2} 2 p^{2}$, i.e. 2 electrons fill the inner shell 1s, which is close to the nucleus and which is irrelevant for chemical reactions, whereas 4 electrons occupy the outer shell of $2 s$ and $2 p$ orbitals [75]. Because the $2 \mathrm{p}$ orbitals $\left(2 \mathrm{p}_{\mathrm{x}}, 2 \mathrm{p}_{\mathrm{y}}\right.$, and $\left.2 \mathrm{p}_{\mathrm{z}}\right)$ are roughly $4 \mathrm{eV}$ higher than the $2 \mathrm{~s}$ orbital, it is energetically favorable to put 2 electrons in the $2 \mathrm{~s}$ orbital and only 2 of them in the $2 p$ orbitals (Figure 2.1). It turns out, however, that in the presence of other atoms, which is carbon atoms in graphene case, it is favorable to excite one electron from the $2 \mathrm{~s}$ to the third $2 p$ orbital, in order to form covalent bonds with the other atoms. The gain in energy from the covalent bond is definitely larger than the $4 \mathrm{eV}$ participated in the electronic excitation. 


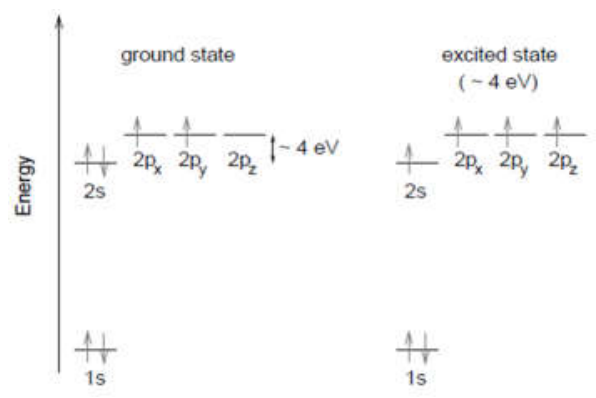

Figure 2.1 Electronic States of Carbon.

In the excited state, therefore, we have quantum-mechanical states, $2 \mathrm{~s}, 2 \mathrm{p}_{\mathrm{x}}, 2 \mathrm{p}_{\mathrm{y}}$, and $2 \mathrm{p}_{\mathrm{z}}$ to make $\mathrm{sp}^{2}$ hybridization in graphene where the $\mathrm{p}_{\mathrm{z}}$ is perpendicular to the $\mathrm{x}-\mathrm{y}$ plane to form a honeycomb lattice structure as shown in Figure 2.2.

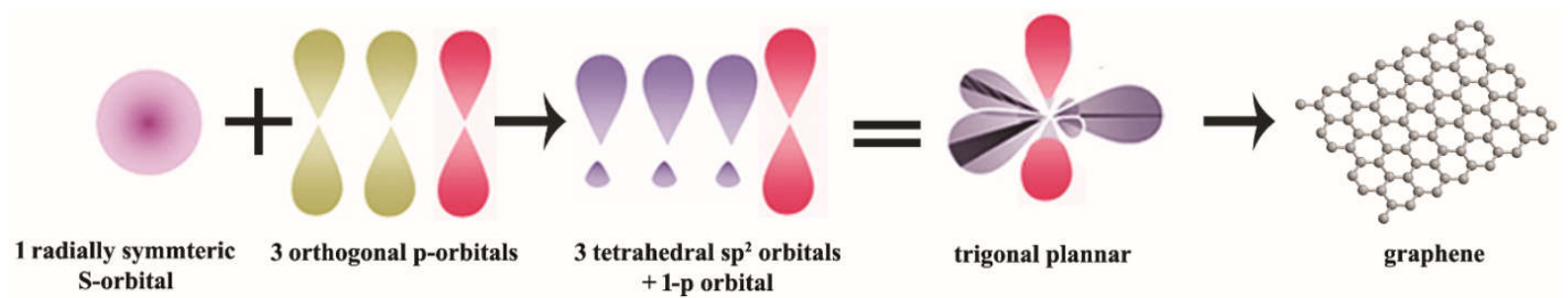

Figure $2.2 \mathrm{sp}^{2}$ hybridization in graphene. Edited and improved using the ref. [76]. Graphene is the name given to a flat monolayer of carbon atoms tightly packed into a twodimensional (2D) honeycomb lattice and is a basic building block for graphitic materials of all other dimensionalities (Figure 2.3). It can be wrapped up into 0D fullerenes, rolled into 1D nanotubes or stacked into 3D graphite because of available van der Waals interaction. 


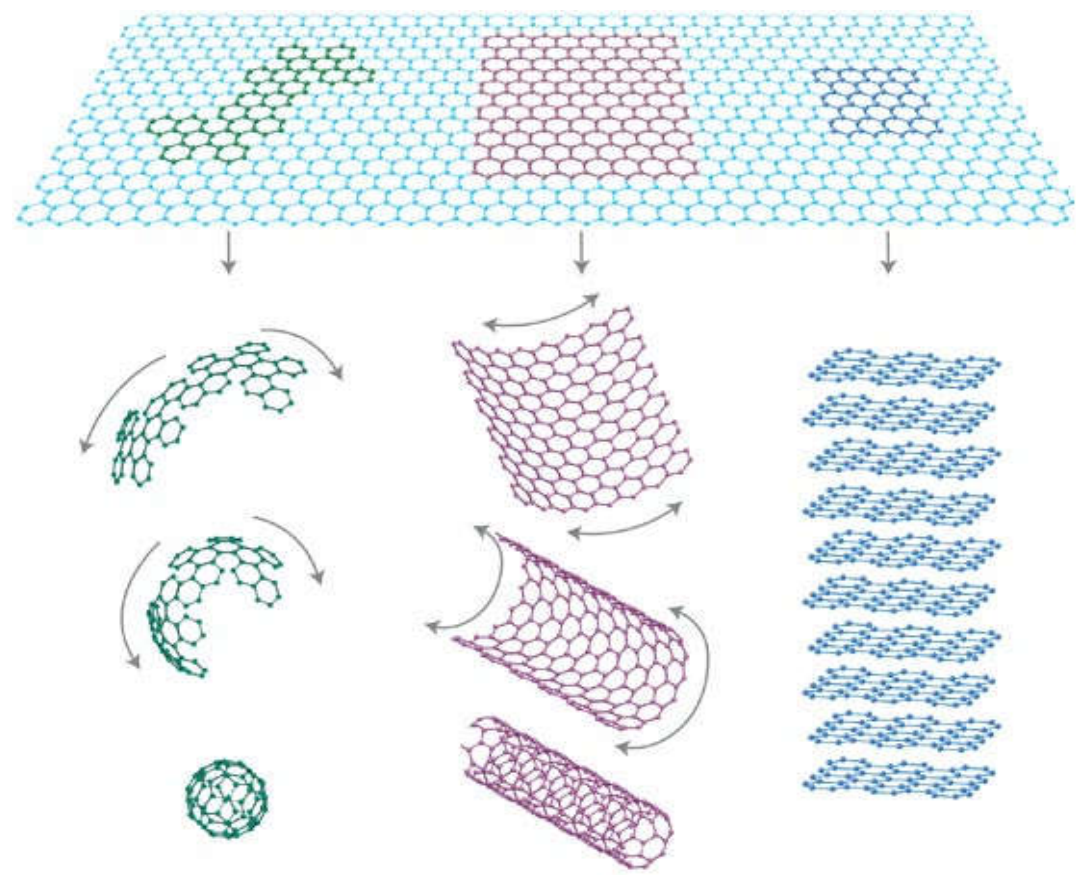

Figure 2.3 Verities of graphitic form.

Graphene has an unusual band structure with a zero bandgap. The energy-momentum relation of a conventional semiconductor material can be expressed as $[77,78]$,

$$
E^{2}=m^{2} C^{4}+p^{2} c^{2}
$$

where $\mathrm{E}=$ total energy, $\mathrm{m}=$ rest mass, $\mathrm{c}=$ velocity of light, $\mathrm{p}=$ momentum. Electrons in ordinary system follow Newton's equations where energy increases in quadratically with increasing velocity $\left({\left.\mathrm{E} \alpha \mathrm{V}^{2}\right)}^{2}\right.$ while for graphene it increases linearly $(\mathrm{E} \alpha \mathrm{V})$ as if electrons in graphene acts as relativistic, massless Dirac participles with high Fermi velocity [77].

Graphene, since the demonstration of its easy isolation by the exfoliation of graphite in 2004 by Novoselov, Geim, and co-workers, has been attracting enormous attention in the scientific community. A single layer or few layers graphene could be synthesized using 
different methods such as exfoliation, chemical synthesis, and epitaxial growth etc. [79]. Exfoliation can be divided
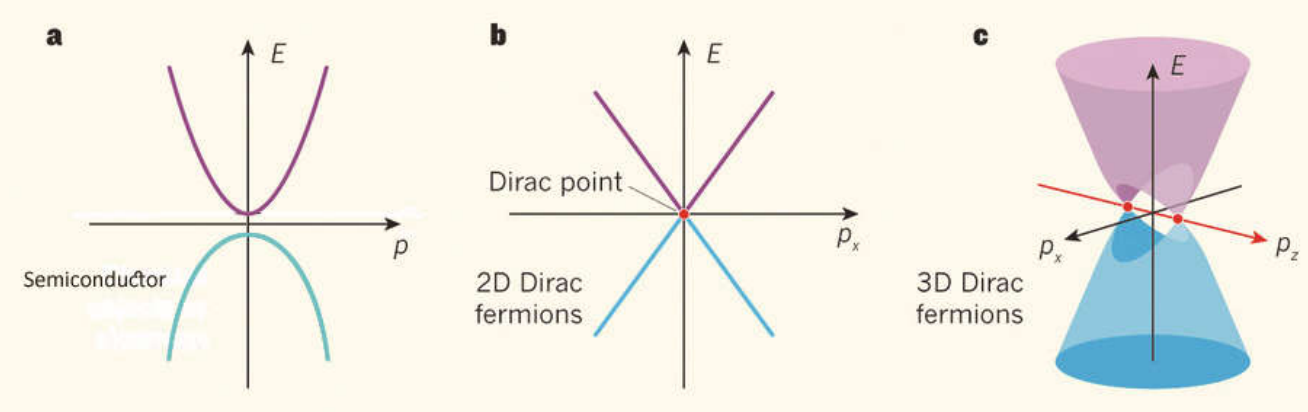

Figure 2.4 (a) Conventional Semiconductors and their electrons exhibit a parabolic relationship between their energy (E) and momentum (p), (b) Two-dimensional (2D) Dirac fermions, such as electrons in graphene, have valence (blue) and conduction (purple) energy bands with a linear energy-momentum relationship. These touch at a point called the Dirac point in the 3D parameter space formed by E, $p_{x}$, and $p_{y}$. Shown here is a 2D slice of the 3D space, (c) A 3D slice of the 4D (E, $\left.p_{x}, p_{y}, p_{z}\right)$ energy-momentum relationship of 3D Dirac fermions such as those discovered [80-83] in $\mathrm{Cd}_{3} \mathrm{As}_{2}$, with two Dirac points along a special high-symmetry axis $\left(\mathrm{p}_{\mathrm{z}}\right)$.

into two sub-categories: mechanical exfoliation and chemical exfoliation. Graphite is essentially stacks of graphene layers attached together using weak van der waals (vdW) force. Graphene could be easily exfoliated from graphite, where the reverse process of stacking is done against the weak vdW force between graphene layers. Very famous scotch tape method is widely used to peel off one or few layers of graphene and transfer on $\mathrm{SiO}_{2} / \mathrm{Si}$ substrate. This mechanical exfoliation process yields high-quality graphene but the scale of production is limited.

Graphene intercalated compounds (GICs) are prepared by intercalating graphene to increase interlayer spacing in chemical exfoliation method, which is then exfoliated into Graphene in the second step by heating or sonication $[19,20]$. 
The most promising and inexpensive process for deposition of reasonably high-quality Graphene is CVD on transition metal substrate in the form of thin foils/films they are polycrystalline in nature and hence the individual grains/crystals are randomly oriented. An RF plasma-enhanced CVD system is widely used to synthesize graphene on various substrates by decomposing hydrocarbons such as methane, ethylene, acetylene, and benzene. A number of graphene layers depend on the hydrocarbon and reaction parameters. CVD growth of graphene has been mainly practiced on copper [21,22] and nickel [23-25] substrates. Analogous to carbon nanotubes graphene can be synthesized by arc discharge of graphite electrodes. The synthesized graphene is in the form of nanosheets or nanoflakes which appear as black powder. Typically arc discharge is carried out under high pressure of hydrogen and helium for pure graphene [84]. The oxidative-exfoliation methods can potentially produce large quantities of graphene oxide (GO), a graphene-like nanosheet which is typically defective and requires additional treatments to reduce it to reduced graphene oxide (RGO)[85]. Reduced graphene oxide (RGO) with properties similar to that of graphene is prepared through chemical, thermal, or electrochemical reduction pathways [20]. Another process for high scale production of graphene includes epitaxial growth on $\mathrm{SiC}(0001)$ in an $\mathrm{Ar}$ atmosphere close to atmospheric pressure provides morphologically superior graphene layers in comparison with vacuum graphitization. Extensive step bunching taking place during processing yields arrays of parallel terraces up to $3 \mu \mathrm{m}$ wide and more than $50 \mu \mathrm{m}$ long whereas high-temperature annealing in vacuum yields graphene layers with small grains $(30-200 \mathrm{~nm})$. The terraces are essentially completely and homogeneously covered with a monolayer of graphene[17]. 
Graphene as a 2D carbon allotrope has drawn tremendous attention worldwide because of its unique properties for electronics, spintronics and surface sciences[1,6]. It is a promising material for high-performance nanoelectronics due to its high carrier concentration $(\sim 2$ $\left.\times 1011 \mathrm{~cm}^{-2}\right)$, mobility $\left(-200,000 \mathrm{~cm}^{2} \mathrm{~V}^{-1} \mathrm{~s}^{-1}\right)$, and stability [2, 86, 87]. But widespread adoption of graphene for electronic devices in particular still faces challenges because it lacks an energy band gap due to band degeneracy at the Dirac point. Breaking translational symmetry or sublattice symmetry could be the option to introduce a band gap in graphene. The application of strain or electric field, chemical edge functionalization and quantum confinement in graphene nanoribbons, nanomesh, decoration with Si-rich two-dimensional islands [88] and the introduction of defects have been used in efforts to induce a band gap [37, 46, 47, 89-96], formation of perforated graphene using black copolymer (BCP) lithography [95], adsorption of patterned hydrogen and water molecules[96, 97].

\subsection{Germanene:}

Despite having unique electronics, spintronics, and surface science properties widespread adoption of graphene for electronic devices still faces challenges because it lacks an energy band gap. The application of strain or electric field, chemical edge functionalization and quantum confinement in graphene nanoribbons, nanomesh, and the introduction of defects have been used in efforts to induce a band gap[37, 51, 90].

In spite of the triumph of graphene, its shortcomings have spurred a quest for other 2D materials that naturally have or can more easily induce a band gap, high mobility or on/off ratio. Two promising 2D materials are silicene and germanene, 2D counterparts of graphene originating from bulk silicon and germanium respectively, as first predicted by 
Takeda and Shiraishi et al. in 1994 [98]. They also envisaged that the planar graphene-like structure of germanene may not exist and may behave like a poor metal. However, the low buckled form of germanene seems to be stable and behaves as a semi-metal. Cahangirov et al. found that the armchair and zigzag nanoribbons appear to be from this low-buckled germanene[13].Q-pang et al. showed that these ribbons exhibit intriguing electronic and magnetic properties[99]. Germanene has the same honeycomb structure as graphene, but the atoms are buckled, resulting in increased stability and improved carrier transport characteristics in comparison to graphene. Moreover, the electronic structure of germanene is quite similar to graphene. The atomic and band structure of germanene are presented in Figure 2.4(a) and 2.4(b), respectively. Puckered structures of germanene are ambipolar and behave like massless Dirac Fermions[13, 14]. Even more interesting is that the Fermi

(a)

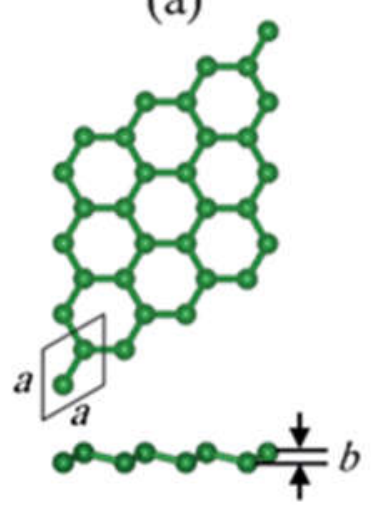

(b)

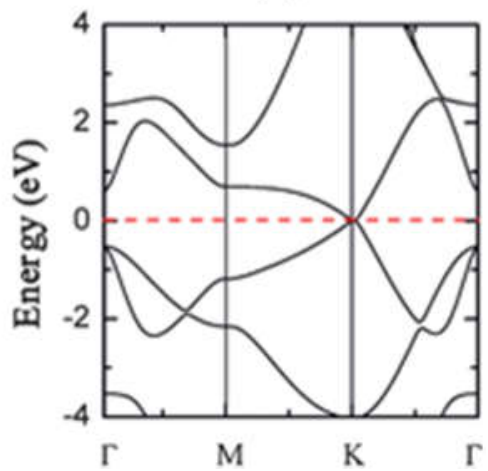

Figure 2.5 (a) The top and side views of the low-buckled germanene sheet, the hexagonal lattice constant, a, and the buckling height, b, are indicated, (b) The band structure of low-buckled germanene with a Fermi level set at zero energy indicated by the red dashed line.

the velocity of germanene is $\sim 5.6 \times 10^{5} \mathrm{~ms}^{-1}$, carrier interactions with phonons are 25 times less than those of graphene which may explain its high carrier mobility [26]. Germanene 
offers large magnetic moment and changes from an AFM to FM state when doped or surface passivated $[99,100]$.

Inspired by these theoretical predictions, germanene synthesis techniques were developed but limited to substrate deposition. Graphene-like germanene sheets have been produced on $\mathrm{Pt}(111), \mathrm{Ge} / \mathrm{Ag}(111)$, and $\mathrm{Al}(111)$ substrates and were also epitaxially and mechanically exfoliated using $\mathrm{GeH}$ synthesis on $\mathrm{SiO} 2$ [27-30]. Moreover, Dávila et al. reported experimental evidence of its synthesis by dry epitaxial growth of germanene on an $\mathrm{Au}(111)$ surface [11]. The thermal stability improved by replacing the $\mathrm{H}$ atom in germanane with a methyl group[101]. Very recently large layers of germanene were synthesized on the band gap material $\mathrm{MoS}_{2}[102]$.

\subsection{Molybdenum Disulfide (MoS2)}

Two-dimensional (2D) materials have been some of the most expansively studied materials in the recent years due to the profusion of uncommon physical phenomena that arise when charge and heat transport are confined quantum-mechanically in a plane. This new endeavor to explore 2D materials, commenced by the work on graphene by Novoselov and Geim in 2004, has led to significant interest in the transition metal chalcogenides (TMCs) due to their simplicity of synthesis and their suitability for nanoelectronics devices [103]. $\mathrm{MoS}_{2}$ is a TMC of particular significance since it possesses a direct bandgap of around 1.8 $\mathrm{eV}$, making it a suitable material for field effect transistor (FET) and optoelectronic applications [54-56]. 


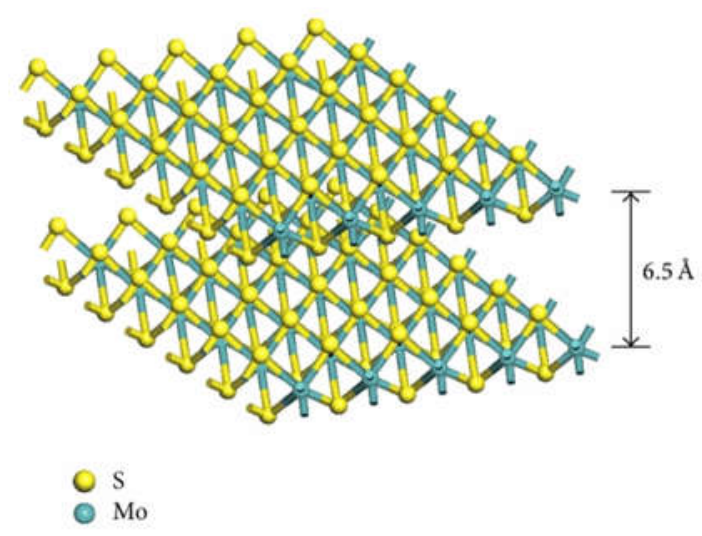

Figure 2.6 $\mathrm{MoS}_{2}$ Structure

$\mathrm{MoS}_{2}$ is a distinctive member of the TMC family. Just like graphene, crystals of $\mathrm{MoS}_{2}$ are comprised of weakly bonded layers vertically stacked together by van der Waals forces. The atoms within each layer are covalently bonded in hexagonally packed $\mathrm{MoS}_{6}$ trigonal prisms, essentially resulting in an S-Mo-S "sandwich" (Figure 2.6). The weak van der Waals interlayer bonding allows for easy micromechanical exfoliation using "scotch-tape method" of large-area, high-quality, atomically thin crystals. Due to notable electronic, optical and mechanical properties of $\mathrm{MoS}_{2}$ has drawn increasing interest, specially for photoelectronic devices.

\subsection{Silicene}

Silicene, a 2D form of silicon which is analogous of graphene, has attracted amassed attention during the recent years. Since 1994, the possibility of low buckled the Si analogs of graphite had been theoretically predicted, there were very few studies on silicene. Then ab initio calculations in 2009 confirmed the existence of dynamically stable silicene with a low buckled structure. Despite the low buckled geometry, silicene has most of the 
outstanding electronic properties of planar graphene such as the "Dirac cone", high Fermi velocity $\left(-10^{5}\right.$ to $\left.10^{6} \mathrm{~ms}^{-1}\right)$ and carrier mobility $\left(-2.57 \times 105 \mathrm{~cm}^{2} \mathrm{~V}^{-1} \mathrm{~s}^{-1}\right)[104,105]$. Silicene has several prominent advantages over graphene: (1) a much stronger spin-orbit coupling, which might be leads realization of quantum spin Hall effect in the experimentally feasible temperature, (2) a superior tunability of the band gap, which is suitbale effective field effect transistor (FET) operating at ambient temperature, (3) valley polarization and more suitability for valleytronics study. Since 2012, different monolayer silicene superstructures have been successfully synthesized on various substrates, including $\operatorname{Ag}(111), \operatorname{Ir}(111)$, $\mathrm{ZrB2}(0001), \mathrm{ZrC}(111)$ and MoS2 surfaces [8, 15, 16, 31, 32]. Multilayer silicene sheets have also been grown on $\operatorname{Ag}(111)$ surface [10]. The experimental accomplishments have inspired researchers to explore the intrinsic properties as well as potential device applications of silicene, including quantum spin Hall effect, quantum anomalous Hall effect, quantum valley Hall effect, superconductivity, band engineering, magnetism, thermoelectric effect, gas sensor, tunneling FET, spin filter, and spin FET, etc. Lately, silicene FET has been fabricated, which shows the predictable ambipolar Dirac charge transport like graphene and paves the way towards silicene-based nanoelectronics [46, $106]$.

\subsection{Comparison of 2D materials}

The comparison of 2D materials represented in Figure 2.7 where germanene and silicene show buckling of $0.737 \AA$ and $0.537 \AA$ respectively while graphene and $\mathrm{MoS}_{2}$ present planar structure. Though germanene and silicene are structurally similar they possess different electronic and magnetic properties because of distinct bond length and buckling. 
The Fermi velocity recorded so far $\sim 2.5 \times 10^{6}, 4.2 \times 10^{5}, \sim 5.6 \times 10^{5}$ and $1.1 \times 10^{6} \mathrm{~ms}^{-1}$ for graphene, silicene, germanene, and $\mathrm{MoS}_{2}$ respectively [26, 104, 107, 108]. While silicene, germanene, and graphene show zero band gap, a 2D form of $\mathrm{MoS}_{2}$ has a direct band gap of $\sim 1.8 \mathrm{eV}[54,56]$. Another kind of $2 \mathrm{D}$ form is hexagonal boron nitride which widely used as an insulating layer for other 2D materials.

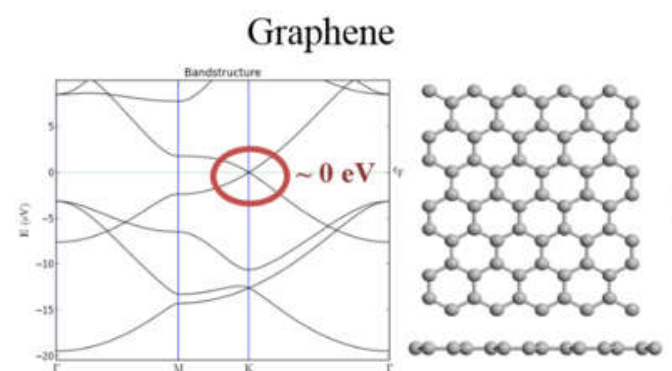

Molybdenum Disulfide $\left(\mathrm{MoS}_{2}\right)$

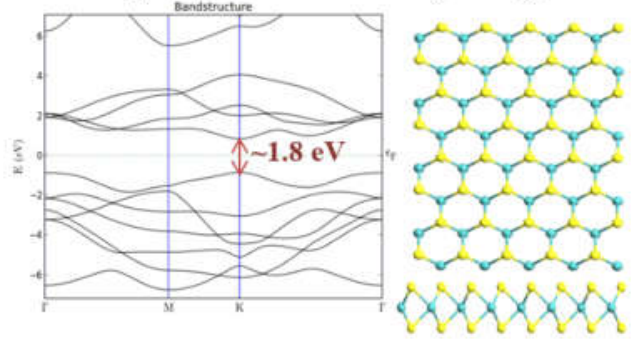

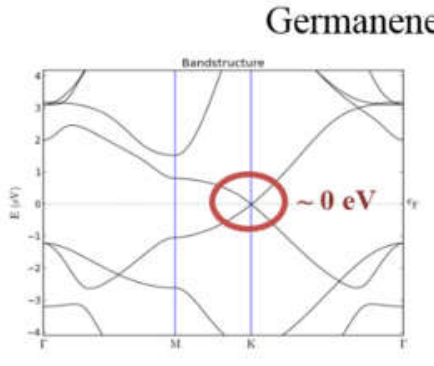

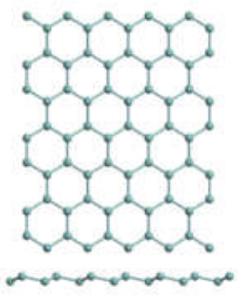

Hexagonal Boron Nitride (h-BN)

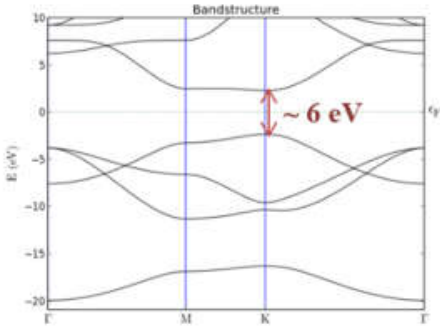

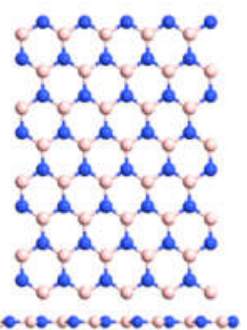

Figure 2.7 Band structure and atomic structure of (a) graphene, (b) germanene, (c) $\mathrm{MoS}_{2}$ and (d) hexagonal boron nitride (h-BN). 


\section{CHAPTER 3}

\section{Methodology}

\subsection{Introduction}

In this dissertation, we employed the first-principles method based on non-equilibrium Green's function (NEGF) combined with density functional theory (DFT). In this chapter, the basic principles behind these powerful tools are introduced.

\subsection{Density Functional Theory}

As we know from quantum mechanics, all information about a given system is contained in its wave function, $\Psi$. This wave function for a single electron moving in a potential energy $V(r)$ is calculated from non-relativistic Schrödinger's equation:

$$
\hat{H} \Psi(r)=\left[-\frac{\hbar^{2} \nabla^{2}}{2 m}+V(r)\right] \Psi(r)=E \Psi(r)
$$

Here, $\hat{\mathrm{H}}$ is the Hamiltonian operator, $r$ is the position vector, $\hbar$ is the reduced Planck constant, $m$ is particle mass, $\nabla^{2}$ is the Laplacian, and $E$ is the energy of state $\Psi$. For more than one electron (multi-body problems) the Schrödinger's equation becomes:

$$
\left[\sum_{i}^{N}\left(-\frac{\hbar^{2} \nabla_{i}^{2}}{2 m}+V\left(r_{i}\right)\right)+\sum_{i \prec j} U\left(r_{i}, r_{j}\right)\right] \Psi\left(r_{1}, r_{2}, \ldots, r_{N}\right)=E \Psi\left(r_{1}, r_{2}, \ldots, r_{N}\right)
$$

Here, $N$ is the number of electrons and $U\left(r_{i}, r_{j}\right)$ is electron-electron interaction. Numerous powerful methods for solving Schrödinger's equation for multi-body problems have been developed; however, it seems impossible to apply them to large and complex systems. DFT provides a way of reducing this problem to three spatial dependencies by presenting precise simplifications and approximations which will be introduced in the following 
sections. The DFT method was first introduced in two influential papers in the 60's [109, 110]. Walter Kohn was awarded with the Nobel Prize in Chemistry in 1998 for his development of the DFT.

\subsubsection{Born-Oppenheimer Approximation}

The Born-Oppenheimer approximation is defined based on the nuclei-electrons interactions. Because the nuclei are considerably more massive than electrons, the response of electrons to the nuclear motion is instantaneousness. It is reasonable to consider the position of the nucleus fixed when calculating the electronic wavefunctions. As a result, the wavefunctions of a molecule can be separated into nuclear and electrons [111]:

$$
\Psi_{\text {total }}=\psi_{\text {electronics }} \times \psi_{\text {nuclear }}
$$

Furthermore, the nuclear degrees of freedom in the Hamiltonian are eliminated:

$$
\hat{H}=T_{e}+V_{n \Leftrightarrow e}+V_{e \Leftrightarrow e}
$$

Here, $V$ and $T$ denote potential energy and kinetic energy, respectively. Moreover, $e$ and $n$ represent electron and nuclear interaction, correspondingly. It is worth to mention that the Born-Oppenheimer approximation alone is not adequate to solve the Schrödinger's equation, and further simplifications are necessary.

\subsubsection{Hohenberg-Kohn Theorems}

The two theorems proved by Hohenberg and Kohn in 1964 [109], make DFT existence possible. The first Hohenberg-Kohn theorem states that the external potential is a unique function of the electron density $\rho(r)$. It means that the ground state wave function $\psi$ and 
total energy $E[(\psi)]$ of a system can be distinctively determined by the ground state electron density:

$$
E[(\psi)]=E[\rho(r)]
$$

The second theorem indicates that the ground state electron density is the exact density that minimizes the total energy. To put it another way, if the Schrödinger's equation is solved for the ground state electron density, the corresponding wave function can be determined. In this way, the unobservable wavefunction is interpreted by the observable electron density which is the number of electrons at a specific location is. As a result, the system can be fully described. Although the Hohenberg-Kohn theorems are tremendously powerful, the ground state density cannot be computed using them.

\subsubsection{Kohn-Sham Equations}

The Kohn-Sham theorem offers an amazing way to compute the electron density by solving the Schrödinger's equation of a fictitious system of non-interacting particles in the form of [110]:

$$
\left[-\frac{\hbar^{2} \nabla^{2}}{2 m}+V_{e f f}(r)\right] \psi_{\alpha}(r)=\varepsilon_{\alpha} \psi_{\alpha}(r)
$$

Here, $\varepsilon_{\alpha}$ is the orbital energy of the corresponding Kohn-Sham orbital, $\psi_{\alpha}$. Furthermore, $V_{\text {eff }}(r)$ represents the Kohn-Sham potential in which the non-interacting particles move and has three contributions:

$$
V_{\text {eff }}(r)=V_{H}(r)+V_{x c}(r)+V_{e x t}(r)
$$


The first two terms are related to the interactions with other electrons. $V_{H}(r)$ is the Hartree potential owing to the mean-field electrostatic interaction, and $V_{X C}(r)$ represents the exchange-correlation potential caused by the quantum mechanical nature of the electrons. The Hartree potential as classical electrostatic potential can be calculated from the electron density:

$$
\nabla^{2} V_{H}(r)=-4 \pi \rho(r)
$$

Additionally, the exchange-correlation potential is:

$$
V_{x c}(r)=-\frac{\delta E_{x c}(\rho)}{\delta \rho(r)}
$$

Here, $E_{x d}(\rho)$ represents the exchange-correlation energy which will be introduced in detail later. The third term, $V_{\text {ext }}(r)$ is associated with any other electrostatic interactions in the system. This term can be caused by the ion potentials (described by norm-conserving pseudopotentials) and electrostatic interactions with the external electrostatic field. Pseudopotential is an attempt to simplify the calculations by replacing the effects of core electrons of an atom with an effective potential. This approximation was first introduced by Hans Hellmann in 1934 [112]. As can be seen in Figure 3.1 [113], the wave function oscillates rapidly in the core electrons region because of the strong ionic potential. By replacing the real potential (red solid line) with a weaker pseudopotential (blue dashed line), a pseudo wave function with a smooth curve is constructed [113]. 


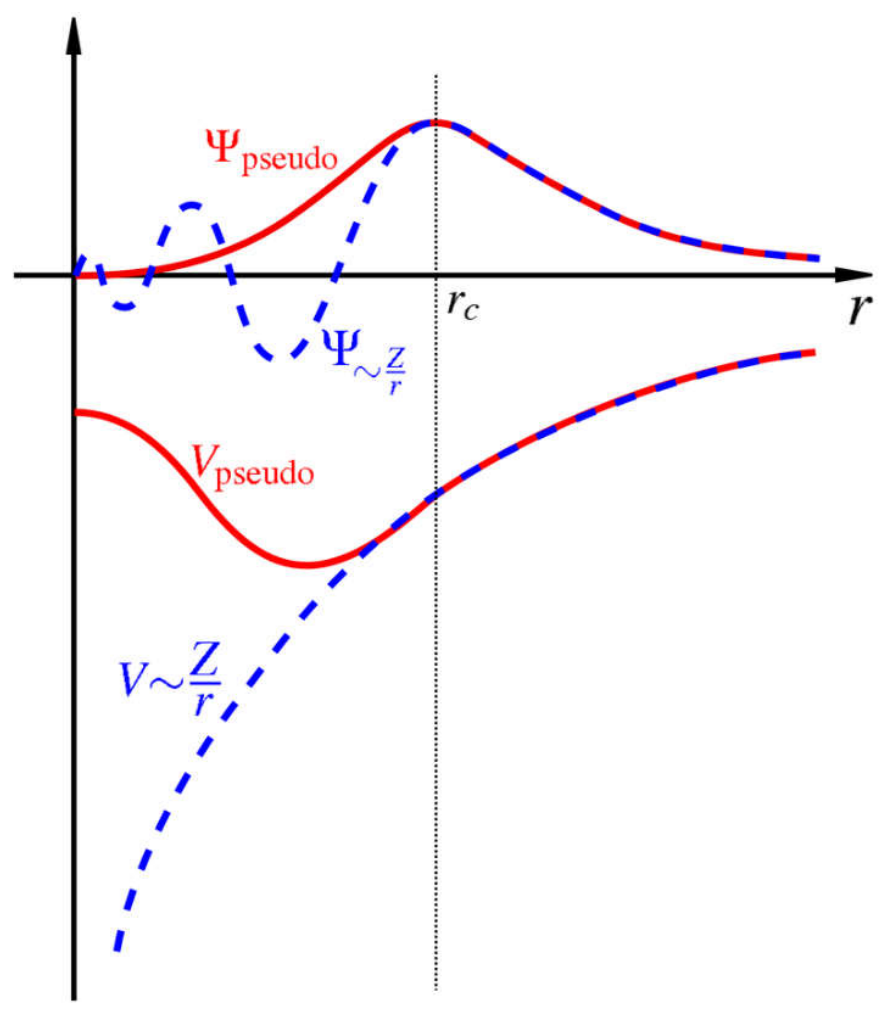

Figure 3.1 A potential and its corresponding wave function of all-electron (blue) compared to that of pseudoelectron (red). Here, $r_{c}$ is the radius at which all-electron and pseudopotential values match [113].

As a result, the Kohn-Sham theorem considers a fictitious system of non-interacting particles within an effective potential to study a real system in which particles interact within an external potential [114].

\subsection{Electron Density}

The electron density of a closed or periodic system with N-particles can be defined based on the occupied eigenstates as:

$$
\rho(r)=\sum_{\alpha=1}^{N}\left|\psi_{\alpha}(r)\right|^{2} f\left(\frac{\varepsilon_{\alpha}-\varepsilon_{f}}{k T}\right)
$$


Here, $f(x)=1 /\left(1+e^{x}\right)$ is the Fermi function, $\varepsilon_{f}$ is the Fermi energy, $T$ is the electron temperature, and $\mathrm{k}$ is the Boltzmann constant. Conveniently, electron density can be presented in term of density matrix $\left(D_{i j}\right)$ :

$$
\rho(r)=\sum_{\alpha} D_{i j} \phi_{i}(r) \phi_{j}(r)
$$

Where $D_{i j}$ is defined by basis set expansion coefficients

$$
D_{i j}=\sum_{\alpha} c_{\alpha i}^{*} c_{\alpha j} f\left(\frac{\varepsilon_{\alpha}-\varepsilon_{f}}{k T}\right)
$$

Moreover, the electron difference density can be calculated by comparing the electron density with a superposition of atom based densities:

$$
\Delta \rho(r)=\rho(r)-\sum_{\mu} \rho^{a t o m}\left(r-r_{\mu}\right)
$$

\subsection{Total Energy}

The Kohn-Sham potential and electron density are self-consistently calculated up until the electron density which minimizes the total energy is found. The total energy of a system as a function of electron density is expressed as follows:

$$
E(\rho)=T(\rho)+E_{H}(\rho)+E_{e x t}(\rho)+E_{x c}(\rho)
$$

Here, $T(\rho)$ is the kinetic energy of non-interacting particles which is described as:

$$
T_{s}(\rho)=\sum_{\alpha=1}^{N} \int d r \psi_{\alpha}^{*}(r)\left(-\frac{\hbar^{2}}{2 m} \nabla^{2}\right) \psi_{\alpha}(r) f\left(\frac{\varepsilon_{\alpha}-\varepsilon_{f}}{k T}\right)
$$

$E_{H}(\rho)$ is the Hartree energy which can be expressed as: 


$$
E_{H}(\rho)=\frac{e^{2}}{2} \int d r \int d r^{\prime} \frac{\rho(r) \rho\left(r^{\prime}\right)}{\left|r-r^{\prime}\right|}
$$

$E_{\text {ext }}(\rho)$ is the energy related to interactions with the pseudopotential ions and other electrostatic external potentials and given by:

$$
E_{e x t}(\rho)=\int d r V_{e x t}(r) \rho(r)
$$

\subsection{Exchange-correlation Energy}

The quantum mechanical effect of the other electrons in the system is included in the exchange-correlation energy term. There are various approximations for the exchangecorrelation energy. The choice of the appropriate functional greatly depends on the system at hand. Experience and benchmarking are required to find the best choice. The local density approximation (LDA) is the lowest rung on Jacob's ladder of approximations [115, 116], in which the exchange-correlational energy depends only on the electronic density at a point in space [110]. The LDA for the exchange-correlation energy is given by:

$$
E_{x c}^{L D A}(\rho)=\int \rho(\vec{r}) \varepsilon_{x c}(\rho(r)) d r
$$

Here, $\varepsilon_{x c}(\rho(r))$ is the exchange-correlation energy of a homogeneous electron gas with a density of $\rho(r))$. Although the exact exchange energy for the homogeneous electron gas, socalled Dirac-Block exchange, can be calculated, the correlation energy needs some approximation to be calculated. To this end, a number of approximations such as PerdewZunger (PZ) [117] and Perdew-Wang (PW) [118], and Vosko-Wilk-Nusair (VWN) [119] have been used. 
The generalized gradient approximation (GGA) is a functional in which both the density and its gradient at each point are considered. With this additional information, GGA is usually more accurate than LDA. The GGA for the exchange-correlation energy is:

$$
E_{x c}^{G G A}(\rho)=\int \rho(\vec{r}) \varepsilon_{x c}(\rho(r), \overrightarrow{\nabla \rho}) d r
$$

Here, $\varepsilon_{x c}(\rho(r), \overrightarrow{\nabla \rho})$ is the exchange-correlation energy as a function of density and its gradient. Different parameterizations of GGA are reported in the literature such as PerdewWang 1991 (PW91) [120], Perdew-Burke-Ernzerhof (PBE) [121], and BLYP (from the name Becke for the exchange part and Lee, Yang and Parr for the correlation part) [122, 123]. Another functional is meta-GGA which uses the Laplacian of the density in addition to the density and its gradient. The Tao-Perdew-Staroverov-Scuseria (TPSS) is a functional of this type [124].

Hybrid functionals is another class of approximations to the exchange-correlation energy that uses a fraction of exact exchange from Hartree-Fock theory as well as exchange and correlation from other sources such as ab initio or empirical. B3LYP (Becke, 3-parameter, Lee-Yang-Parr) is one the most frequently used functionals of this type $[125,126]$. The exchange-correlation energy of B3LYP functional is expressed as:

$$
E_{x c}^{B 3 L Y P}=E_{x}^{L D A}+a_{0}\left(E_{x}^{H F}-E_{x}^{L D A}\right)+a_{x}\left(E_{x}^{G G A}-E_{x}^{L D A}\right)+E_{c}^{L D A}+a_{c}\left(E_{c}^{G G A}-E_{c}^{L D A}\right)
$$

Here, $a_{0}, a_{x}$, and $a_{c}$ are $0.20,0.72$, and 0.81 , respectively. $E_{x}^{D A A}$ and $E_{x}^{G G A}$ are the exchange energies of LDA-VWN [119] and GGA-Beke 88 [122], correspondingly. $E_{c}^{D A A}$ and $E_{c}^{G G A}$ 
represent the correlation energies of LDA-VWN and GGA-Beke 88 functionals, respectively. $E_{c}^{H F}$ is the Hartree-Fock energy which can be expressed as:

$$
E_{x}^{H f}=-\frac{1}{2} \sum_{i, j} \iint \psi_{i}^{*}\left(r_{1}\right) \psi_{j}^{*}\left(r_{1}\right) \frac{1}{r_{12}} \psi_{i}\left(r_{2}\right) \psi_{j}\left(r_{2}\right) d r_{1} d r_{2}
$$

\subsection{Self-consistent Loop in DFT}

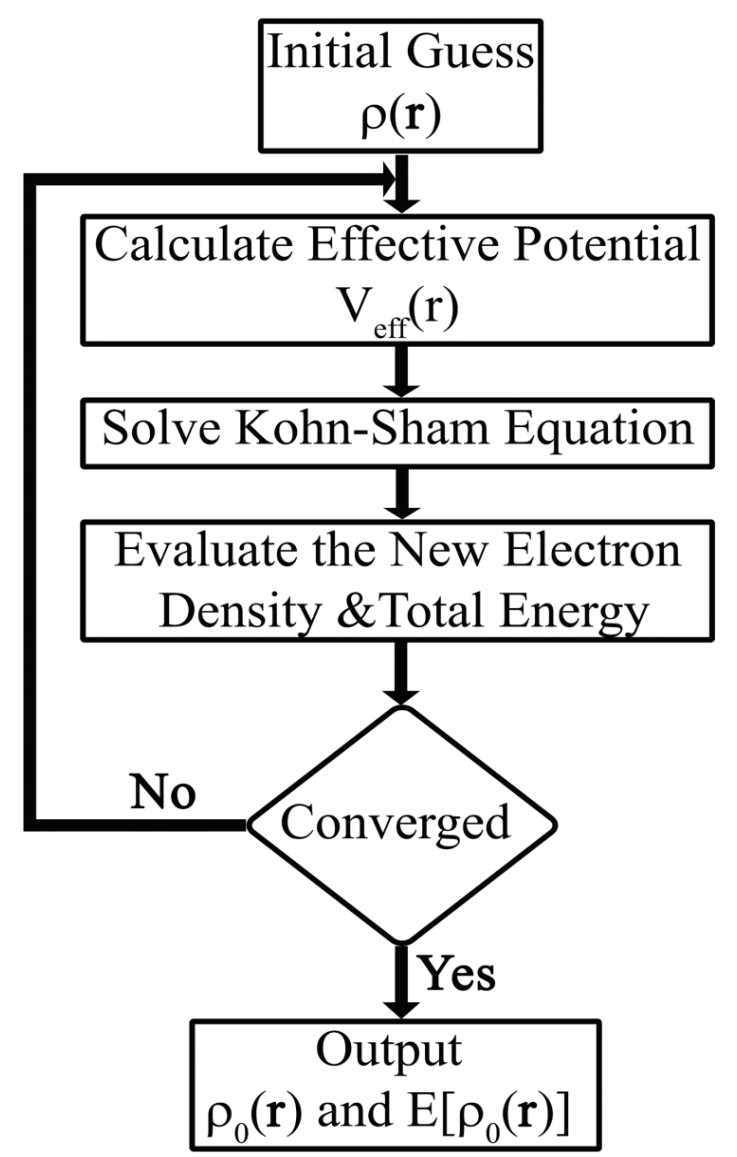

Figure 3.2 A flow chart of the iteration scheme in DFT method for calculating electron density and total energy.

A schematic representing a self-consistent loop in DFT method to obtaining electron density is illustrated in Figure 3.2. First, an initial guess for the electron density is assumed. By repetitively solving the Kohn-Sham equations using the appropriate exchange- 
correlation functional, we can obtain a self-consistent solution that minimizes the total energy, resulting in a desired ground state electron density.

\section{7 van der Waals Contribution in DFT}

Materials, as well as molecules, are stabilized by primary interatomic bonds, such as covalent, ionic or metallic bonds. In this section, we discuss the secondary bonding by vander-Waals (vdW) interactions. Van-der-Waals forces are usually weaker than for example covalent interatomic forces. Of particular importance for this work is their role for the interlayer binding of graphite and few-layer graphene. However, all the exchangecorrelation functionals introduced in Section 3.5 do not include the long range vdW tail. Different scientific communities define vdW forces differently; throughout this work, the term vdW energy or dispersion energy will be used to describe the energy contributions originating from induced dipole - induced dipole interactions.

The corrections developed by Grimme and his coworkers [127, 128]. They add an additional term $E_{\text {disp }}$ to the DFT so that the total energy $E_{D F T}$ account for van der Waals interactions:

$$
E_{D F T+D}=E_{D F T}+E_{d i s p}
$$

The D2 and D3 corrections differ in the way the term $E_{\text {disp }}$ is evaluated. 


\subsubsection{D2 correction}

In the $\mathrm{D} 2$ correction [127] the $E_{\text {disp }}$ term includes only two-body energies and is given by an attractive semi-empirical pair potential $\mathrm{V}^{\mathrm{PP}}$ which accounts only for the lowest-order dispersion term:

$$
E_{\text {disp }}=-S_{6} \sum_{A<B} V^{p p}\left(Z_{A}, Z_{B}, Z_{C}\right)
$$

where $R_{A}\left(R_{B}\right)$ and $R_{A B}$ are the atomic number of atom $A$ (atom $B$ ) and the interatomic distance, respectively. The global scaling parameter $\mathrm{S}_{6}$ has been fitted to reproduce the thermochemical properties of a large training set of molecules and depends on the exchange-correlation functional employed.

The pair potential between two atoms with atomic numbers A and B separated by a distance $\mathrm{R}_{\mathrm{AB}}$ is:

$$
V^{p p}=\frac{\sqrt{C_{6}^{A} C_{6}^{B}}}{R_{A B}^{6}} f\left(R_{A B}\right)
$$

Where $f\left(R_{A B}\right)=\frac{1}{1+e^{-d_{6}\left[\frac{R_{A B}}{R_{0}^{A}+R_{0}^{B}}\right]}}$

is a damping function and $\mathrm{C}_{6}$ and $\mathrm{R}_{0}$ are element-specific parameters. Typically, the damping factor $\mathrm{d}_{6}$ is set to 20 . 


\subsection{Transport Properties}

In order to investigate the electronic structure and electronic transport for systems connected to semi-infinite electrodes under a voltage bias, the DFT combined with nonequilibrium Green's function (NEGF) method is used. The detailed explanation of this method can be found in the literature [129-132]. The device system is divided into three regions: two electrode regions (left and right) and a central region, as illustrated in Figure 3.3. The system is periodic in the left and right electrodes. To match the effective potential of a central region with bulk electrodes, the perturbation of the scattering region should be screened out. To this end, a sufficient fraction of the electrode regions should be repeated in the central region. As a result, the effective potential of the outermost part of the central region is similar to that of bulk electrodes.

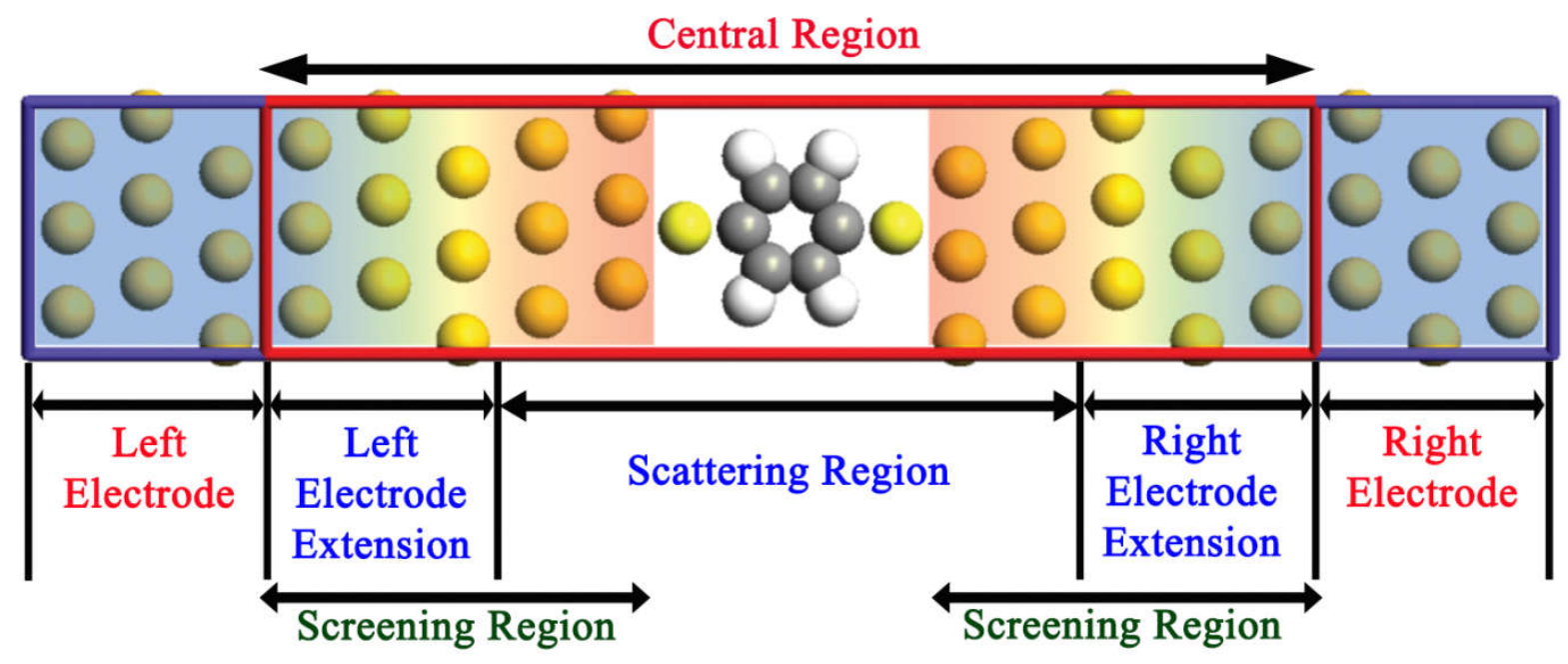

Figure 3.3 Geometry of a device configuration with two electrodes. The system is periodic in the left and right electrodes. The central region is seamlessly connected to the electrodes. The parts of the central region where the atoms have the arrangement same as electrodes are called the left and right electrode extension.

To calculate the non-equilibrium electron distribution in the central region, the NEGF method is employed. First, the density matrix is divided into left and right contributions: 


$$
D=D^{L}+D^{R}
$$

where $D^{L(R)}$ is calculated using NEGF theory by [130]

$$
D^{L(R)}=\int \rho^{L(R)}(\varepsilon) f\left(\frac{\varepsilon_{\alpha}-\mu_{L(R)}}{k_{B} T_{L(R)}}\right) d \varepsilon
$$

Where $\rho^{L(R}(\varepsilon)$, the spectral density matrix, is

$$
\rho^{L(R)}(\varepsilon) \equiv \frac{1}{2 \pi} G(\varepsilon) \Gamma^{L(R)}(\varepsilon) G^{\dagger}(\varepsilon)
$$

Here $\Gamma^{L(R)}$ is the broadening function of the left (right) electrode which defined as

$$
\Gamma^{L(R)}=\frac{1}{i}\left(\sum^{L(R)}-\left(\sum^{L(R)}\right)^{\dagger}\right)
$$

where $\Sigma^{L(R}$, the left (right) electrode self-energy, is

$$
\sum^{L(R)}=V_{S / L(R)} g_{L(R)} V_{L(R) / S}
$$

Here, $g_{L(R)}$ is the surface Green's function for the semi-infinite electrodes and $V_{L(R) / S}=V_{S / L(R)}^{+}$ are the coupling matrix elements between electrodes and the scattering region. Furthermore, the key quantity to calculate is G, the retarded Green's function matrix,

$$
G(\varepsilon)=\frac{1}{\left(\varepsilon+i \delta_{+}\right) S-H}
$$

where $\delta_{+}$is an infinitesimal positive number. $\mathrm{S}$ and $\mathrm{H}$ are the overlap and Hamiltonian matrices of the entire system, respectively. The Green's function is only required for the central region and can be calculated from the Hamiltonian of the central region by adding the electrode self-energies:

$$
G(\varepsilon)=\left[\left(\varepsilon+i \delta_{+}\right) S-H-\sum^{L}(\varepsilon)-\sum^{R}(\varepsilon)\right]^{-1}
$$

The transmission amplitude $t_{k}$, defines the fraction of a scattering state $k$ propagating through a device. The transmission coefficient at energy $\varepsilon$ is obtained by summing up the transmission from all the states at this energy,

$$
T(\varepsilon)=\sum_{k} t_{k}^{\dagger} t_{k} \delta\left(\varepsilon-\varepsilon_{k}\right)
$$


The transmission coefficient may also be obtained from the retarded Green's function using

$$
T(\varepsilon)=G(\varepsilon) \Gamma^{L}(\varepsilon) G^{\dagger}(\varepsilon) \Gamma^{R}(\varepsilon)
$$

At $T_{R}=T_{L}=O$ (electron temperature) the conductance is determined by the transmission coefficient at the Fermi Level,

$$
C(\varepsilon)=G_{0} T(\varepsilon)
$$

where $G_{0}=2 e^{2} / h$ is the quantum conductance, in which $e$ is the electron charge and $h$ is Planck's constant. 


\section{CHAPTER 4}

\section{Edge-functionalized germanene nanoribbons: impact on electronic and magnetic properties}

\subsection{Overview}

Germanene exhibits extremely high mobility, massless fermion behavior, and strong spinorbit coupling drawing tremendous interest for high-performance devices. It has a buckled two-dimensional structure, but no intrinsic energy band gap and structural stability required for logic and switching devices. Application of a perpendicular electric field, surface adsorption, confinement of an armchair nanoribbon structure and edge functionalization are methods used to open a band gap. Edge functionalization of armchair germanene nanoribbons (AGeNRs) has the potential to achieve a range of band gaps. The edge atoms of AGeNRs are passivated with hydrogen $(-\mathrm{H}$ and $-2 \mathrm{H})$ or halogen $(-\mathrm{F},-\mathrm{Cl},-\mathrm{OH},-2 \mathrm{~F},-2 \mathrm{Cl})$ atoms. Using density functional theory calculations, we found that edge-functionalized AGeNRs had band gaps as small as $0.012 \mathrm{eV}$ when functionalized by $-2 \mathrm{H}$ and as high as $0.84 \mathrm{eV}$ with $-2 \mathrm{~F}$. Formation energy studies revealed that AGeNRs produced a more stable structure under fluorine functionalization. Simulation results suggest that the electronic structure of germanene is similar to graphene and silicene. A spin-polarized density functional theory (DFT) study of electronic and magnetic properties of pristine, chemically functionalized and doped AGeNRs and zigzag nanoribbons (ZGeNRs) was performed. Formation energy studies revealed that the Ge atoms at the edge of the ribbon prefer to be replaced by impurity atoms. Doping can change the semiconducting behavior of AGeNRs to metal due to the half-filled band making it useful for negative differential resistance 
(NDR) devices. In the case of ZGeNRs, single N or B doping transformed them from antiferromagnetic (AFM) semiconducting to ferromagnetic (FM) semiconductor or half-metal. These magnetic and electronic properties make edge-functionalized doped AGeNRs and ZGeNRs promising for use in field effect transistors (FETs) and spintronics. Finally, energy band gap tuning of AGeNRs and ZGeNRs using edge functionalization may open a new route to integrate germanene in logic and high-performance switching devices.

\subsection{Introduction}

Graphene as a 2D carbon allotrope has drawn considerable attention worldwide because of its unique properties for electronics, spintronics and surface science $[1,6]$. But widespread adoption of graphene for electronic devices still faces challenges because it lacks an energy band gap. The application of strain or electric field, chemical edge functionalization and quantum confinement in graphene nanoribbons, nanomesh, and the introduction of defects have been used in efforts to induce a band gap[37, 51,90].

Despite the success of graphene, its shortcomings have spurred a quest for other 2D materials that naturally have or can more easily induce a band gap, high mobility or on/off ratio. Two promising $2 \mathrm{D}$ materials are silicene and germanene, 2D counterparts of graphene originating from bulk silicon and germanium respectively, as first predicted by Takeda and Shiraishi et al. in 1994 [98]. They also envisaged that the planar graphene-like structure of germanene may not exist and may behave like a poor metal. However, the low buckled form of germanene seems to be stable and behaves as a semi-metal. Cahangirov et al., found that the armchair and zigzag nanoribbons appear to be from this low-buckled germanene[13]. Q-pang et al. showed that these ribbons exhibit intriguing electronic and 
magnetic properties [99]. Germanene has the same honeycomb structure as graphene, but the atoms are buckled, resulting in increased stability and improved carrier transport characteristics in comparison to graphene. Moreover, the electronic structure of germanene is quite similar to graphene. Puckered structures of germanene are ambipolar and behave like massless Dirac Fermions [13, 14]. Even more interesting is that the Fermi velocity of germanene is $\sim 5.6 \times 10^{5} \mathrm{~ms}^{-1}$, carrier interactions with phonons are 25 times less than those of graphene which may explain its high carrier mobility [26]. Germanene offers large magnetic moment and changes from an AFM to FM state when doped or surface passivated $[99,100]$.

Inspired by these theoretical predictions, germanene synthesis techniques were developed but limited to substrate deposition. Graphene-like germanene sheets have been produced on $\mathrm{Pt}(111), \mathrm{Ge} / \mathrm{Ag}(111)$, and $\mathrm{Al}(111)$ substrates and were also epitaxially and mechanically exfoliated using $\mathrm{GeH}$ synthesis on $\mathrm{SiO}_{2}$ [27-30]. Moreover, Dávila et al. reported experimental evidence of its synthesis by the dry epitaxial growth of germanene on an $\mathrm{Au}(111)$ surface [11]. The thermal stability improved by replacing the $\mathrm{H}$ atom in germanane with a
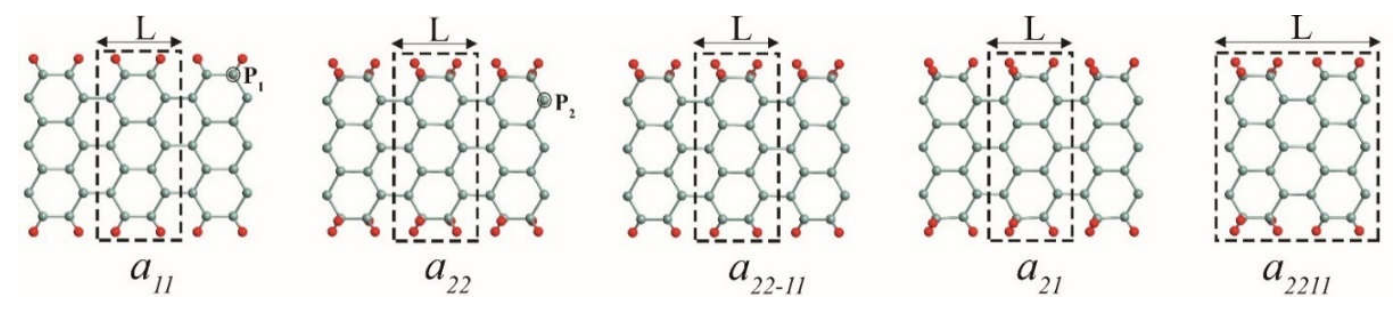

Figure 4.1 Graphical models of edge-functionalized 7-AGeNRs. L is the periodic length of the structure. The primitive supercell is marked by a dashed box. The cyan and red balls represent germanium and functional addends $(\mathrm{H}, \mathrm{F}, \mathrm{Cl}, \mathrm{Br}$, and $\mathrm{I})$, respectively. $\mathrm{P}_{1}$ and $\mathrm{P}_{2}$ are the preferred position of doping with boron $(B)$ or nitrogen $(\mathrm{N})$. 
methyl group [101]. Very recently large layers of germanene were synthesized on the band gap material $\mathrm{MoS}_{2}[102]$.

On the basis of density functional theory (DFT) calculations, Pang et al. studied completely hydrogenated armchair GeNR (AGeNRs) and zigzag GeNRs (ZGeNRs) [99]. They found that the band gap of AGeNRs decreases with increasing ribbon width while oscillates with a period of three. ZGeNRs exhibit antiferromagnetic (AFM) semiconducting behavior but changed to ferromagnetic (FM) semiconducting when doped with a single B or $\mathrm{N}$ atom which is useful for spintronic applications [133, 134].

Doping is one method for inducing ferromagnetism in the ZGeNRs while retaining its semiconducting behavior. It was also established that edge states dramatically modify the electronic and magnetic properties of GNRs [50, 135-141]. While doping changes the magnetic state of NRs, edge functionalization (such as using hydrogen and halogens) is an attractive method to alter the electronic properties of GeNRs. Due to halogen functionalization, a large band gap is found because of the stronger spin-orbit coupling (SOC) of $\sigma$ orbitals around the Fermi level instead of weaker SOC. The coupling of the $\mathrm{p}_{\mathrm{xy}}$ orbitals of Ge and heavy halogen atoms forming the $\sigma$ orbitals contribute towards large band gap opening [142].

Despite numerous attractive features in germanene and similarity of electronic properties to graphene, its semi-metallic zero band gap still requires engineering to open it. Applying electric field, chemisorption of adatom species, introducing periodic nanoholes, doping and 
edge functionalization are possible routes to open and tune a band gap in silicene and germanene at the $\mathrm{K}$ point. Edge functionalization as a tool for tuning the band gap in nanoribbons has been studied [46, 50, 93, 130, 143-148]. But those studies included only hydrogenation and did not include a comprehensive range of edge types for germanene nanoribbons.

Here, a detailed theoretical study of the influence of edge-functionalization, using hydrogen $(-\mathrm{H}$ and $-2 \mathrm{H})$ or halogen $(-\mathrm{F},-\mathrm{Cl},-2 \mathrm{~F},-2 \mathrm{Cl})$ atoms is presented and a full range of edge types by means of density functional theory (DFT) calculations combined with non-equilibrium green's function (NEGF) carried out using ATOMISTIX TOOLKIT (ATK) $[121,130,148,149]$. An overview is given of the influence of these edge-functional groups attached to different germanene nanoribbon structures of varying widths ranging from 6 to 19, focusing on structural and electronic ribbon properties, especially energy band gap. Additionally, the dependence of armchair GeNR energy band gap value on functionalization and ribbon width is explored to better classify the calculated results. Additionally, the impacts of doping on the stability, electronic and magnetic properties of different edge-functionalized GeNRs with hydrogen or halogen atoms are also explored. It was found that doping could increase the sensitivity capabilities of 2D nanomaterials [150, 151]. These results can help guide future experimental synthesis and theoretical studies in tuning the electronic and magnetic properties of germanene nanoribbons for electronic material and devices. 


\subsection{Model and Method}

Germanene nanoribbons (GeNRs) were investigated with edge functionalization by hydrogen $(-\mathrm{H}$ and $-2 \mathrm{H})$, halogen $(-\mathrm{F},-\mathrm{Cl},-2 \mathrm{~F},-2 \mathrm{Cl})$ or hydroxide $(-\mathrm{OH})$ atoms. Within the framework of DFT, the structure's geometry of GeNRs are optimized using a maximum atomic force of $0.01 \mathrm{eV} / \AA$ and stress of $0.0005 \mathrm{eV} / \AA^{3}$ using a Hartwigsen-GoedeckerHutter (HGH) pseudopotential tier 4 basis set in the ATK 2014 code and Generalized Gradient Approximation (GGA) of Perdew-Burke-Ernzerhof (PBE) functional was used for exchange-correlation. A grid mesh cut-off energy of $75 \mathrm{Ha}$ and $1 \times 1 \times 21 \mathrm{k}$-points for the integration of the first Brillouin zone is taken. A vacuum space of $15 \AA$ is considered for each side of the supercell to suppress any interaction between structures and its periodic image. On the basis of the equilibrium structures, the electronic and magnetic properties are calculated with $1 \times 1 \times 121$ k-points. The Ge-Ge bond length of $2.4 \AA$ and buckling distance of $0.676 \AA$ were obtained after geometric optimization, which are in agreement with previous results $[14,99]$.

\subsection{Results and Discussions}

The edge-functionalized AGeNRs structures studied are presented in Figure 4.1. To distinguish a number of functionalized edge configurations of GeNRs " $a$ " is used for armchair and "z" for zigzag [134]. Furthermore, the subscript denotes the number of edge atoms attached to each of the edge germanium atoms along the periodic direction. To study electronic and magnetic properties, 6-19 atoms wide AGeNRs are considered. In this report, detailed theoretical predictions of AGeNR's electronic properties have been analyzed for different functionalized atoms such as hydrogen, hydro-oxide, and halogens. 
Finally, for the B or N doping study of AGeNRs or ZGeNRs, a 7 atoms wide nanoribbon is chosen.

\subsection{Armchair Germanene Nanoribbon}

\subsubsection{Stability of AGeNRs}

Three common forms of edge hydrogenation and halogenation are studied: mono-atom on both edges $\left(\mathrm{a}_{11}\right)$, di-atoms on both edges $\left(\mathrm{a}_{22}\right)$, and di-atoms on one edge and mono-atom on the other edge ( $\left.\mathrm{a}_{22-11}\right)$, as shown in Figure 4.1. In addition, the $a_{21}$ structure is considered in which germanium atoms are periodically attached to mono-hydrogen (-halogen) and dihydrogen (-halogen) and $\mathrm{a}_{2211}$ structure which is a mix of $\mathrm{a}_{22}$ and $\mathrm{a}_{11}$ structures. Germanene nanoribbons (GeNRs) of width $\sim 7.2 \AA$ were used to model decoupled hydrogen-terminated germanene edges. Ribbon segments in one unit cell consist of germanium atoms and hydrogen atoms. The edge formation energy is then calculated using

$$
E_{\text {edge }}=\left(E_{\text {ribbon }}-n_{\mathrm{Ge}} \times E_{\mathrm{Ge}}-\left(n_{\mathrm{H}} / 2\right) \times E_{H_{2}}\right) / 2 L
$$

Here, $E_{\text {ribbon }}$ is the total internal energy of the nanoribbon segment in a unit cell. $L$ is the length along the ribbon axis of the repeated nanoribbon segment with two similar opposed germanene hydrogenated edge configurations. $E_{\mathrm{Ge}}$ is the energy of a germanium atom in a perfect germanene sheet, $E_{\mathrm{H} 2}$ gives the total internal energy of an isolated molecule [152]. $n_{\mathrm{Ge}}$ and $n_{\mathrm{H}}$ are the number of germanium and hydrogen atoms, respectively. The negative values of $E_{\text {dge }}$ correspond to an exothermic process in perfect vacuum conditions around freestanding GeNRs. The edge formation energies, band gap energy, and magnetic edge states of various AGeNR configurations are listed in Table 4.1. 
Table 4.1 Formation energy $E_{f}(\mathrm{eV})$, band gap $E_{\mathrm{g}}(\mathrm{eV})$, magnetic state, the energy difference between the FM and AFM state $\triangle \mathrm{E}_{\mathrm{FM}-\mathrm{AFM}}(\mathrm{eV})$, and spin-polarized band gap $(\mathrm{eV})$ for $\mathrm{N}$ - and B-doped armchair edge GeNRs functionalized with hydrogen and fluorine.

\begin{tabular}{|c|c|c|c|c|c|c|c|}
\hline \multirow{2}{*}{$\begin{array}{l}\text { Edge } \\
\text { Type }\end{array}$} & \multirow{2}{*}{$\begin{array}{l}\text { Edge } \\
\text { Atom }\end{array}$} & \multirow{2}{*}{ Dopant } & \multirow{2}{*}{$\begin{array}{c}\mathrm{E}_{f} \\
(\mathrm{eV} / \mathrm{A})\end{array}$} & \multirow{2}{*}{$\begin{array}{c}\text { Band gap } \\
(\mathrm{eV})\end{array}$} & \multirow{2}{*}{$\begin{array}{l}\text { Magnetic } \\
\text { State }\end{array}$} & \multicolumn{2}{|c|}{ Magnetic Edge State } \\
\hline & & & & & & $\Delta \mathrm{E}(\mathrm{meV})$ & Band gap (eV) \\
\hline \multirow{4}{*}{$a_{11}$} & $\mathrm{H}$ & $\mathrm{N}$ & -0.522 & 0.0 & FM & -0.08 & - \\
\hline & $\mathrm{H}$ & $\mathrm{B}$ & -4.163 & 0.0 & NM & - & - \\
\hline & $\mathrm{F}$ & $\mathrm{N}$ & +2.654 & 0.0 & AFM-S & +0.01 & Up (0.1362) (in) - Down (0.4392) (d) \\
\hline & $\mathrm{F}$ & $\mathrm{B}$ & -4.737 & 0.0 & FM-S & -0.01 & Up $(0.4254)(d)-$ Down $(0.3932)(d)$ \\
\hline \multirow{4}{*}{$a_{22}$} & $\mathrm{H}$ & $\mathrm{N}$ & +0.142 & 0.00 & NM & - & - \\
\hline & $\mathrm{H}$ & $\mathrm{B}$ & -4.663 & 0.00 & NM & - & - \\
\hline & $\mathrm{F}$ & $\mathrm{N}$ & -0.473 & 0.00 & NM & - & - \\
\hline & $\mathrm{F}$ & B & -4.043 & 0.389 (d) & NM & - & - \\
\hline
\end{tabular}

$a$ : armchair, z: zigzag, subscripts: the number of functional atoms attached to Ge edge atom, NM: non-magnetic, FM: ferromagnetic, AFM: antiferromagnetic, S: semiconductor, d: direct band gap, in: indirect band gap, $\Delta \mathrm{E}: \mathrm{E}_{\mathrm{FM}}-\mathrm{E}_{\mathrm{AFM}}$

Edge functionalization is used for stabilization as the AGeNRs are unstable $(+0.1953$ $\mathrm{eV} / \AA$ ) and hydrogenated structures with $a_{22}$ configurations provide more stability than bare ones but with edge energies close to zero rather than negative. One can consider the hydrogenated structures metastable. Among all of the configurations, the $a_{22}$ fluorinated edge functionalized ribbons are the most stable structure $(-1.88 \mathrm{eV} / \AA)$.

To study the effect of experimental conditions such as gas pressure and temperature, on the stability of functionalized GeNRs, the calculated edge formation energies are compared to the chemical potential $\left(\mu_{\mathrm{H} 2}\right)$ of the edge functionalizing atoms. The edge free energy $\mu_{\mathrm{H}}$ $(T, p)$ is calculated as follows $[153,154]$

$$
\gamma_{\mathrm{H}}(T, p)=E_{\text {edge }}-\frac{1}{2} \times \rho_{\mathrm{H}} \times\left(\mu_{\mathrm{H}_{2}}(T, p)-E_{\mathrm{H}_{2}}\right)
$$


Here, $\rho_{\mathrm{H}}=n_{\mathrm{H}} / 2 L$ is the edge atom density. For instance, edge molecule chemical potential $\mu_{\mathrm{H} 2}$ depends on the gas pressure and temperature according to the formula noted below $[153,154]$

$$
\mu_{\mathrm{H}_{2}}(T, p)=E_{\mathrm{H}_{2}}+\mu_{\mathrm{H}_{2}}^{o}(T, p)
$$

The zero reference state of $\mu_{\mathrm{H} 2}(T, p)$ is chosen to be the total energy of an isolated edge molecule $E_{\mathrm{H} 2}$ at $T=0$, i.e., $\mu_{\mathrm{H} 2}(0, p)=E_{\mathrm{H} 2} \equiv 0$. With respect to this reference, the pressure and temperature dependence contribution to the chemical potential is then given by ${ }^{44-45}$

$$
\mu_{H_{2}}^{o}(T, p)=H^{o}(T)-H^{o}(0)-T S^{o}(T)+k_{B} T \ln \left(p / p^{o}\right)
$$

Here, $H^{o}\left(S^{o}\right)$ is the enthalpy (entropy) of hydrogen molecule at the pressure $p^{o}=1 \mathrm{bar}$, which is obtained from Ref. [155]. One must define the thermodynamic limits of hydrogen's chemical potential. The upper limits are defined by the chemical potential at which the hydrogen molecule is formed, which is equal to the total energy of an isolated molecule at $T=0 \mathrm{~K}$.

$$
\max \left[\mu_{\mathrm{H}_{2}}(T, p)\right]=E_{\mathrm{H}_{2}}
$$

The lower limits are chosen for those molecule's potentials that are accessible experimentally at very high temperature $(900 \mathrm{~K})$.

$$
\min \left[\mu_{\mathrm{H}_{2}}(900, p)\right]=E_{\mathrm{H}_{2}}+\mu_{\mathrm{H}_{2}}^{o}(900, p)
$$




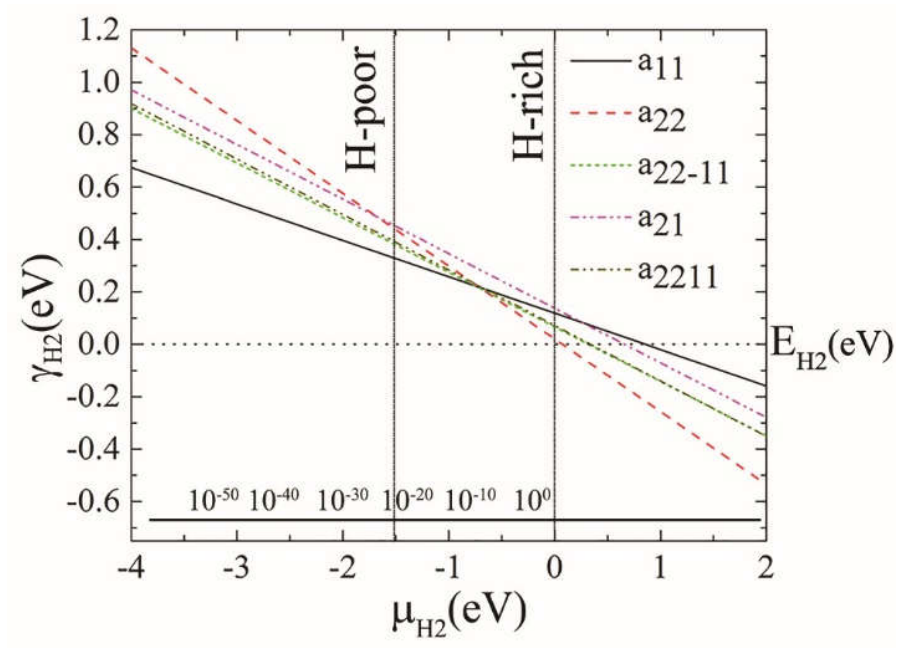

Figure 4.2 Edge free energy $\gamma_{\mathrm{H} 2}$ of hydrogenated GeNR edge structures versus hydrogen chemical potential $\mu_{\mathrm{H} 2}$, using $\mathrm{E}_{\mathrm{H} 2}$ as the zero reference. The allowed range of hydrogen chemical potential is indicated by vertical solid lines. The bottom inset axis represents pressure, in bar, of the molecular $\mathrm{H}_{2}$ corresponding to $\mathrm{T}=300 \mathrm{~K}$.

The magnitude of the second term for hydrogen molecules at a pressure of 1 bar using ref. [155], is $-1.52 \mathrm{eV}$. Consequently, the allowed range of the chemical potential, considering $E_{\mathrm{H} 2}$ as the zero reference, for the hydrogen molecule is

$$
-1.52<\mu_{\mathrm{H}_{2}}(T, p)-E_{\mathrm{H}_{2}}<0
$$

The variations of relative edge formation energy with respect to the chemical potential of, for example, the $\mathrm{H}_{2}$ molecule, using $E_{\mathrm{H} 2}$ as the zero reference, for different AGeNRs configurations are considered and shown in Figure 4.2. Under Hpoor conditions with $\mu_{\mathrm{H} 2}<-1.419$, $\mathrm{a}_{11}$ is feasible for AGeNRs. On the other hand, when $\mu_{\mathrm{H} 2} \geq-1.419$, the a 22 structure becomes more stable than other configurations. Remarkably, under ambient conditions, i.e., $300 \mathrm{~K}$ and $5 \times 10^{-7}$ bar $\left(\mu_{\mathrm{H} 2}=-0.694\right.$ $\mathrm{eV}), \mathrm{a}_{22}$ has minimum energy. It's worthwhile to mention that fluorinated $\mathrm{a}_{22}$ edge structure is always the most stable structure. 


\subsubsection{Electronic properties of AGeNRs}

The variations of the band gap and effective mass of AGeNRs for different widths are shown in Figure 4.3 when edge-functionalized with mono and di-hydrogen. A period of three variations in band gap is clearly observed in Figure 4.3(a), revealing that larger band gaps are obtained with smaller ribbon widths because the quantum confinement effect

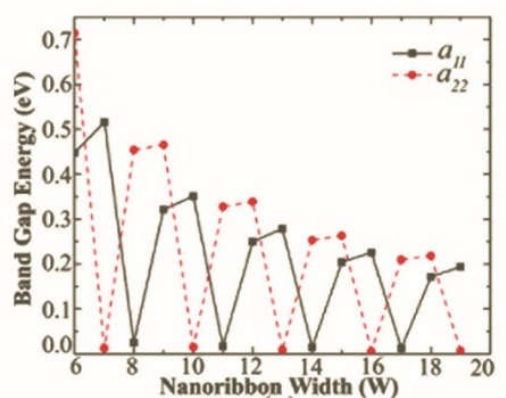

(a)

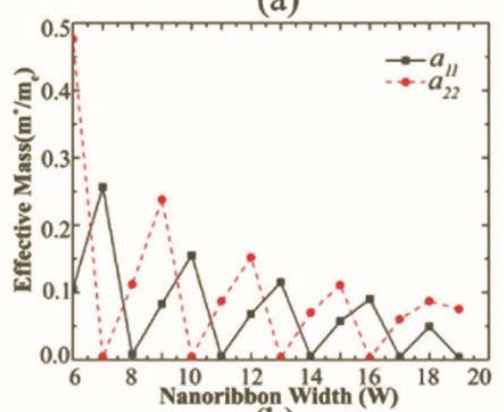

(b)

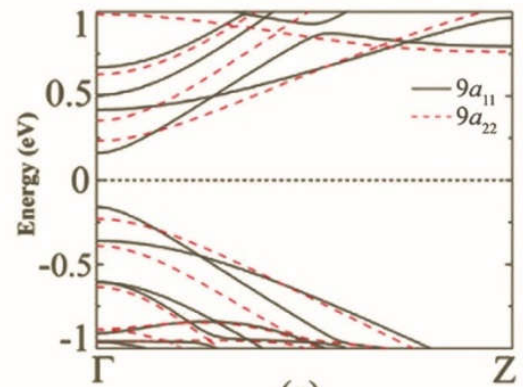

(c)

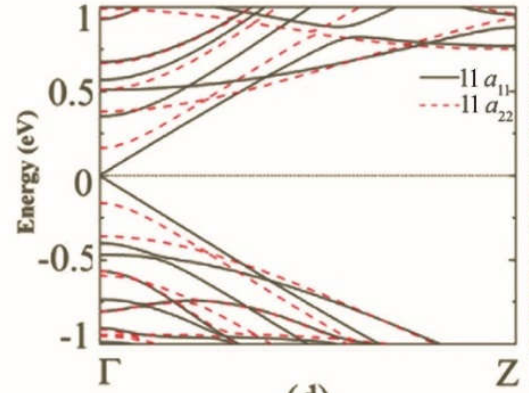

(d)

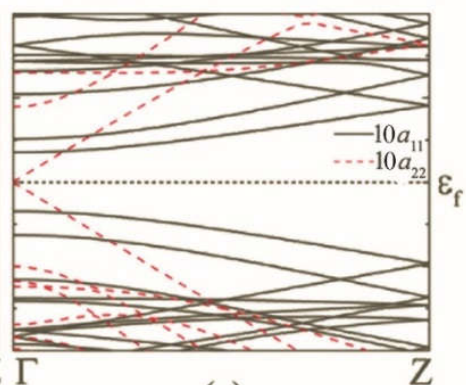

(e)

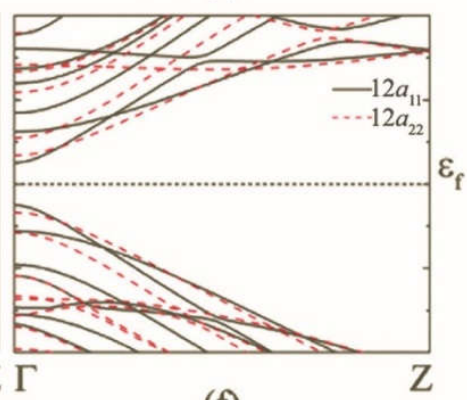

(f)

Figure 4.3 (a) Band gap variation of AGeNR with mono- and di-hydrogen edge functionalization and (b) effective mass variation with ribbon width for mono and dihydrogen edge functionalization of AGeNRs. Band Structure of (c) 9, (d) 10, (e) 11 and (f) 12 atoms wide hydrogen functionalized armchair germanene nanoribbon. The Fermi level $\left(\varepsilon_{\mathrm{f}}\right)$ is set at zero.

is strongly dependent on the nanoribbon width. At the same time, it is found that dihydrogenating a wider ribbon resulted in the same band gaps as smaller monohydrogenated AGeNRs $[50,93,99,147]$. On the other hand, di-hydrogenating AGeNRs offer a stable structure with the same band gap for a larger ribbon width compared to mono- 
hydrogenated edge functionalization. A maximum band gap of $0.714 \mathrm{eV}$ for $6 \mathrm{AgeNR}-2 \mathrm{H}$ and minimum of $0.007 \mathrm{eV}$ for $19 \mathrm{AGeNR}-2 \mathrm{H}$ was found. A similar variation is found in the effective masses of mono and di-hydrogen functionalization.

From Figure 4.3(c-f), the band structure of germanene nanoribbons with hydrogenated edges shows a periodicity of three. This periodicity is classified into three different families where the band gaps are very small for members of the $N_{\mathrm{a}}=3 k+2$ group, moderate for those of the $N_{\mathrm{a}}=3 k$ and large for $N_{\mathrm{a}}=3 k+1$ group. Similar periodicity is also seen for the dihydrogenated case, but this time the band gap follows $N_{\mathrm{a}}=3 k+1<N_{\mathrm{a}}=3 k+2<N_{\mathrm{a}}=3 k . \quad 10$ $\mathrm{a}_{11}$, which belongs to the $3 k+1$ group, shows the highest band gap of $0.35 \mathrm{eV}$ when monohydrogenated but shifted to di-hydrogenated at 11-a 22. This means that almost the same band gap of mono hydrogenated ribbon can be achieved by di-hydrogenation of a wider ribbon which also offers more stability to the structure $[50,93]$. The same periodicity behavior in band gap holds true for hydroxide group edge functionalization.

Both Figure 4.4(a) and (c) show the band gap variation of AGeNRs with ribbon width. It is clear that despite being functionalized by fluorine and chlorine, they still retain the socalled family behavior seen in hydrogenated AGeNRs. The band gap periodicity in fluorine-functionalized germanene nanoribbons show the same behavior as hydrogenated GeNRs. But this time the band gap is reduced drastically with increasing ribbon width because quantum confinement is substantially reduced due to electron saturation by the larger fluorine atom with a mix of $\mathrm{sp}^{2}$ and $\mathrm{sp}^{3}$ bonds providing better structural stability. 
Fluorine, as opposed to hydrogen, which is covalently bonded with germanium to occupy the lowest electronic band leaving Ge-Ge bond unchanged, has higher Pauling electronegativity $(3.98 \mathrm{eV})$ than that of germanium $(2.01 \mathrm{eV})$ making

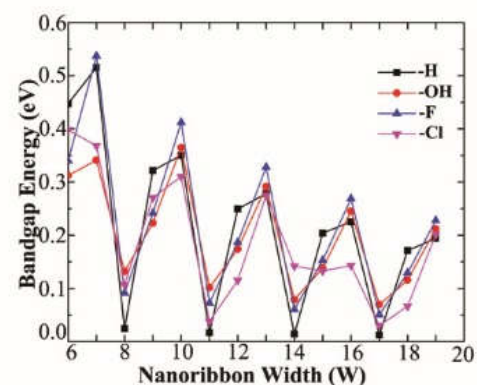

(a)

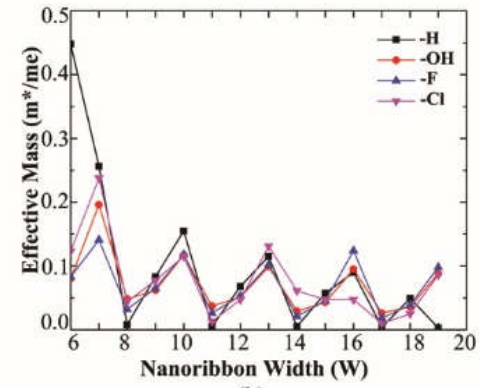

(b)

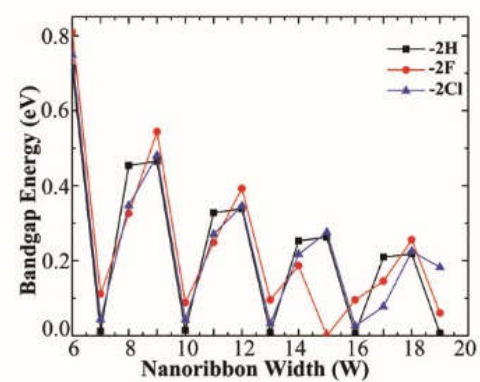

(c)

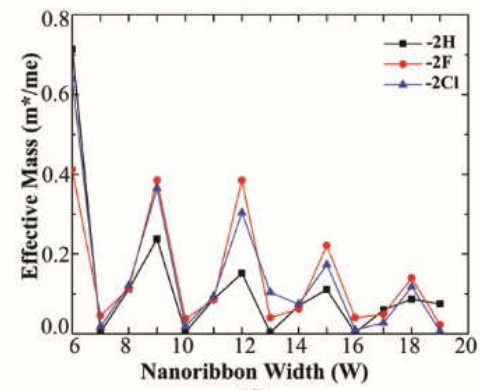

(d)

Figure 4.4 Ribbon width vs band gap (eV) for (a) mono-edge and (b) di-edge functionalization. Effective mass variation of electron with ribbon width for (c) mono- and (d) di- edge functionalization of AGeNRs.

Ge-F bond ionic. Also, a maximum band gap of $0.809 \mathrm{eV}$ was found when the ribbon width was at its lowest, 6 atoms wide and fluorinated. Similar results were found for Ge$\mathrm{Cl}$ bond characteristics. The values of Pauling electronegativity of $\mathrm{X}(=\mathrm{F}, \mathrm{Cl})$ are 3.98 and 3.16, respectively, much larger than germanium.

The van der Waals radiuses of hydrogen and halogens $(\mathrm{X}=\mathrm{F}, \mathrm{Cl})$ are 1.20, 1.47 and 1.75 $\AA$ r respectively $[156,157]$. Ge-Ge bond length $(2.4 \AA)$ in a perfect AGeNRs is almost double 
that of hydrogen's van der Waals radius implying that steric hindrance can be ignored in hydrogenated AGeNRs. But steric hindrance becomes acutely and prominently stronger with increasing atomic number of halogens due to increased van der Waals radius. Ionic characteristic of Ge-X bond for larger functional groups like halogens reduces electron density in the bonding orbital of Ge, deteriorating the interaction between $\mathrm{Ge}-\mathrm{Ge}$ bond. Slightly deviated periodicity in band gap was found

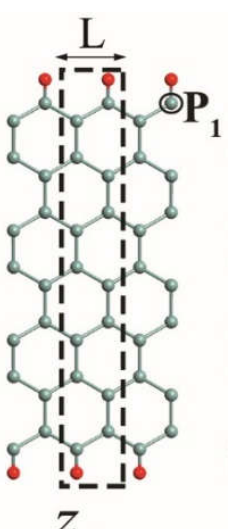

$z_{11}$

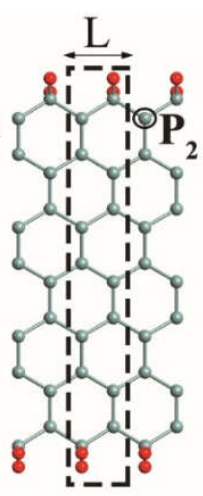

$z_{22}$

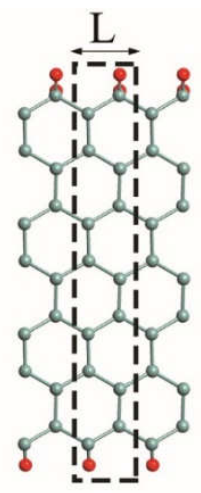

$z_{2-1}$

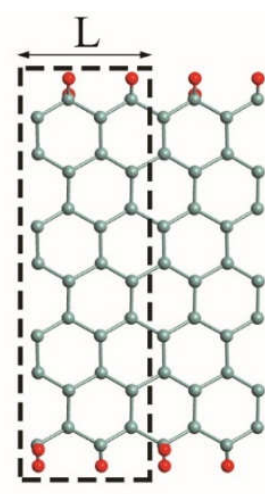

$z_{21}$

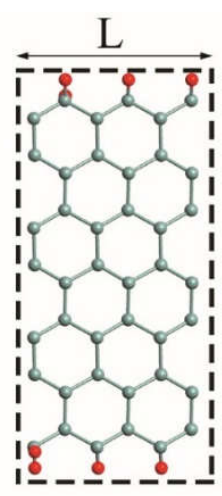

$z_{211}$

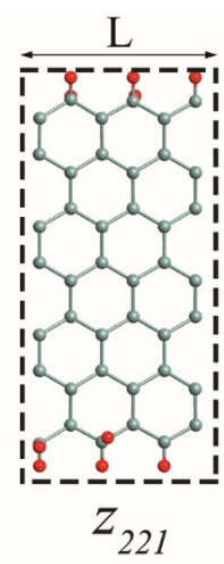

$z_{221}$

Figure 4.5 Structural configurations of edge-functionalized, along with periodic length L, GeNRs studied. The primitive supercell is marked by a dashed black box. The cyan and red balls represent $\mathrm{Ge}$ and functional addends $(\mathrm{H}, \mathrm{F}, \mathrm{Cl}, \mathrm{Br})$, respectively. $\mathrm{P}_{1}$ and $\mathrm{P}_{2}$ are the preferred position of doping with boron $(\mathrm{B})$ or nitrogen $(\mathrm{N})$.

while GeNRs were functionalized with chlorine. As the ribbon width increases, effective mass decreases dramatically and approaches almost the same value with increasing ribbon width as seen in Figure 4.4(b) and (d). The decreasing effective mass offers higher mobility but at the same time, the band gap decreases due to the loss of quantum confinement in the wider ribbon. Most of the edge atom functionalized configurations offer low effective masses for those greater than 9 atoms wide ribbons. Studies show that $\mathrm{N}_{\mathrm{a}}$-AGeNRs (where 
$\left.\mathrm{N}_{\mathrm{a}}=6-19\right)$ are non-magnetic (NM) semiconductors and exhibit direct band gaps at the $\Gamma$ point in the BZ [99].

\subsection{Zigzag Germanene Nanoribbon:}

\subsubsection{Stability of ZGeNRs}

Without any edge passivation ZGeNR is highly unstable $(+0.243 \mathrm{eV} / \AA)$. Various forms of edge functionalization such as hydrogen or halogens can be considered to improve the stability of ZGeNRs. Familiar structures, for instance, $\mathbf{z}_{11}, \mathrm{z}_{22}$, and $\mathrm{z}_{2-1}$ are considered along with some other possible forms of edge functionalization of ZSiNRs, including $\mathrm{z}_{21}$, $\mathrm{z}_{211}$, and $\mathrm{z}_{221}$, as seen in Figure 4.5.

Table 4.2 Edge formation energy $E_{\text {edge }}(\mathrm{eV})$, band gap $E_{\mathrm{g}}(\mathrm{eV})$, magnetic state, the energy difference between the FM and AFM state $\Delta \mathrm{E}_{\mathrm{FM}-\mathrm{AFM}}(\mathrm{eV})$, total magnetic moment $\mathrm{M}_{\text {total }}$ $(\Delta \mathrm{B})$, and spin-polarized band gap $(\mathrm{eV})$ for $\mathrm{z}_{11}$ and $\mathrm{z}_{22}$ zigzag edge GeNRs functionalized with hydrogen and halogens

\begin{tabular}{|c|c|c|c|c|c|c|c|}
\hline \multirow{2}{*}{$\begin{array}{l}\text { Edge } \\
\text { Type }\end{array}$} & \multirow{2}{*}{$\begin{array}{l}\text { Edge } \\
\text { Atom }\end{array}$} & \multirow{2}{*}{$\begin{array}{c}\text { Eedge } \\
(\mathrm{eV} / \AA ̊ \AA)\end{array}$} & \multirow{2}{*}{$\begin{array}{c}\text { Band Gap } \\
(\mathrm{eV})\end{array}$} & \multirow{2}{*}{$\begin{array}{c}\text { Magnetic } \\
\text { State }\end{array}$} & & \multicolumn{2}{|l|}{ Magnetic Edge State } \\
\hline & & & & & $\begin{array}{l}\Delta \mathrm{E} \\
(\mathrm{meV}) \\
\end{array}$ & Band Gap (eV) & Moment $(\mu \mathrm{B})$ \\
\hline \multirow{4}{*}{$\mathrm{z}_{11}$} & Bare & +0.243 & 0.00 & AFM-S & +14.98 & 0.3274 (d) & - \\
\hline & $\mathrm{H}$ & +0.127 & 0.00 & AFM-S & +11.36 & 0.2626 (d) & 0.00 \\
\hline & $\mathrm{F}$ & -0.730 & 0.00 & AFM-S & +7.73 & $0.2325(\mathrm{~d})$ & 0.00 \\
\hline & $\mathrm{Cl}$ & -0.351 & 0.00 & AFM-S & +2.49 & 0.2226 (d) & 0.00 \\
\hline \multirow{4}{*}{$z_{22}$} & Bare & +0.243 & 0.00 & AFM-S & - & - & - \\
\hline & $\mathrm{H}$ & +0.107 & 0.00 & AFM-S & +0.023 & 0.2833 (in) & 0.00 \\
\hline & $\mathrm{F}$ & -1.665 & 0.00 & NM & 0.0 & 0.00 & 0.00 \\
\hline & $\mathrm{Cl}$ & -0.843 & 0.00 & NM & 0.0 & 0.00 & 0.00 \\
\hline
\end{tabular}

$a$ : armchair, $z$ : zigzag, subscripts: the number of functional atoms attached to Ge edge atom, NM: non-magnetic, FM: ferromagnetic, AFM: antiferromagnetic, S: semiconductor, d: direct band gap, in: indirect band gap, $\Delta \mathrm{E}$ : $\mathrm{E}_{\mathrm{FM}}-\mathrm{E}_{\mathrm{AFM}}$

The edge free energies, band gap, and magnetic states of various ZGeNRs are listed in

Table 4.2. Among all possible edge hydrogenation forms of ZGeNRs, the $Z_{21}(+0.082$ 
$\mathrm{eV} / \AA$ ) is found to be the most stable. However, it is about 4 times less stable than the most stable hydrogenated AGeNR ( $\left.\mathrm{a}_{22}\right)$. The order of structural stability for different configurations of $Z$ GeNRs is $Z_{21}>Z_{211}>Z_{221}>Z_{22}>Z_{11}>Z_{\text {bare. }}$. The possible reason is that $\mathrm{Ge}$ atoms prefer to accept a mix of $\mathrm{sp}^{3}$ hybridizations with $\mathrm{sp}^{2}$ in germanene; therefore, a mix of mono and dihydrogenation can increase the stability of the structures. In addition, similar to AGeNRs, halogenating would increase the stability of the ZGeNRs. The stability of fluorinated $Z_{22}(-1.66 \mathrm{eV} / \AA ̊)$ is more stable than the hydrogenated $Z_{22}(0.107 \mathrm{eV} / \AA \AA)$.

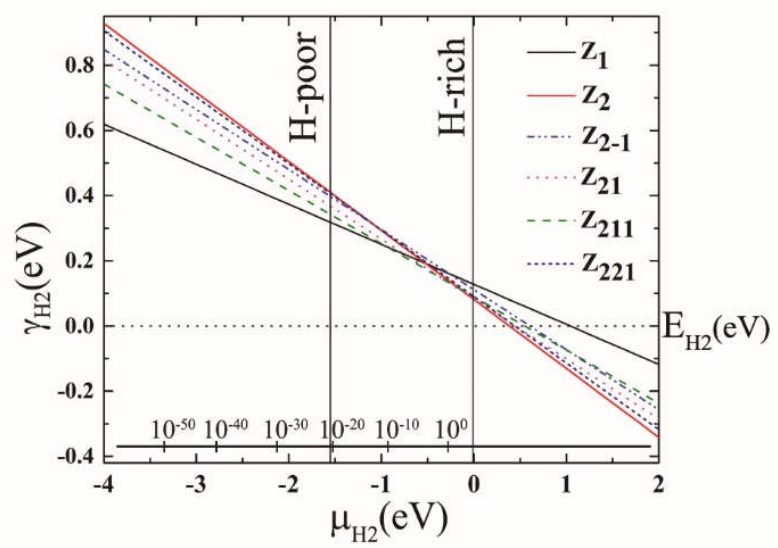

Figure 4.6 Edge free energy $\gamma_{\mathrm{H} 2}$ of different hydrogenated ZGeNRs edge structures versus hydrogen chemical potential $\mu_{\mathrm{H} 2}$, using $\mathrm{E}_{\mathrm{H} 2}$ as the zero reference. The allowed range of hydrogen chemical potential is indicated by vertical solid lines. The bottom inset axis represents pressure, in bar, of the molecular $\mathrm{H}_{2}$ corresponding to $\mathrm{T}=300 \mathrm{~K}$.

Similar to AGeNRs, the stability of halogenated ZGeNRs decreases with increasing atomic number of halogen atoms. In order to account for the experimental conditions, the Gibbs free energies of the aforementioned hydrogenated edge structures are calculated and plotted in Figure 4.6 For low hydrogen chemical potential $\mu_{\mathrm{H} 2}<-1.5 \mathrm{eV}$, the $Z_{11}$ has minimum 
energy. When $-1.5 \mathrm{eV} \leq \mu_{\mathrm{H} 2}<-0.5 \mathrm{eV}$, several different edge structures have almost the same amount of energy. Further increase of $\mu_{\mathrm{H} 2}$ up to $+2.0 \mathrm{eV}$ finds $Z_{22}$ most favorable.

\subsubsection{Electronic and magnetic properties of ZGeNRs}

We studied many configurations of ZGeNR structures. But we present those that are experimentally viable taking into consideration the formation energies while retaining interesting electronic and magnetic properties. Out of those, 7 atoms wide ZGeNRs with mono edge $\left(Z_{11}\right)$ and di-edge $\left(Z_{22}\right)$ structures are presented. Three different magnetic states, NM, FM and AFM of the ZGeNRs are considered. Comparing the total energies of these three states, it is found that the AFM state for the $Z_{11}$ is $80.24 \mathrm{meV}$ and $11.36 \mathrm{meV}$ more favorable than the NM and FM states respectively; thus, similar to ZGNRs, the AFM state is the ground state for ZGeNRs. Figure 4.7(b) shows the band structure of $Z_{11}$ in its ground state (AFM) and it is semiconducting $(0.2626 \mathrm{eV})$ in both (spin-up and spin-down) channels, while two other excited states are metallic. All other $Z_{11}$ configurations while terminated with halogens have the same characteristics as the hydrogenated ones but those structures are comparatively more stable than hydrogen terminated $Z_{11}$ according to their edge free energies. The most stable structure found was terminated by fluorine $(-0.73$

$\mathrm{eV} / \AA ̊$ ). Interestingly, $\mathrm{Z}_{22}$ halogenated structures are metallic in all three states except the hydrogenated (AFM semiconducting, $0.2441 \mathrm{eV}$ ) one which retains same characteristics as $Z_{11}$. 

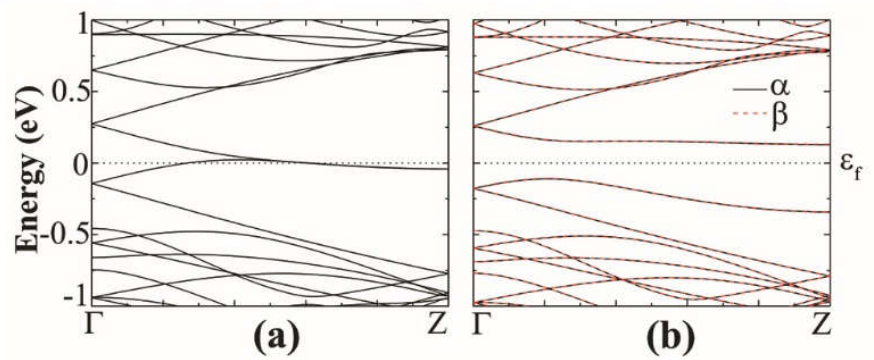

Figure 4.7 Band structure of hydrogenated $Z_{11}$ in the (a) NM and (b) AFM states. $\alpha$ (black solid line) and $\beta$ (red dashed line) represent spin-up and spin-down bands, respectively.

\subsection{Armchair germanene nanoribbons doped by $N$ or $B$ atoms}

The effects of doping on band gap as well as magnetic properties of GeNRs with $\mathrm{N}$ or B impurity have been investigated. Theoretically, it is possible to replace the Ge atoms in different sites of the nanoribbon using a single $\mathrm{N}$ or B. Formation energy $\left(E_{\mathrm{f}}\right)$ of the nanoribbon with an $\mathrm{N}$ or $\mathrm{B}$ impurity is calculated to further examine which site is experimentally more likely to occur,

$$
E_{\mathrm{f}}=E_{\text {doped }}-\left(E_{\text {pristine }}-(m+n) E_{\mathrm{Ge}}+m E_{\mathrm{N}}+n E_{\mathrm{B}}\right)
$$

Here, $E_{\text {doped }}$ and $E_{\text {pristine }}$ are the total energy of doped GeNRs with N or B impurity and the total energy of pristine GeNR, respectively. The $E_{\mathrm{Ge}}, E_{\mathrm{N}}$ and $E_{\mathrm{B}}$ are the total energy of free Ge, $\mathrm{N}$, and $\mathrm{B}$ impurity, respectively. $m$ and $n$ are the number of $\mathrm{N}$ and $\mathrm{B}$ impurities in the doped GeNR, respectively. The smaller value of $E_{\mathrm{f}}$ specifies that the particular atomic structure is experimentally feasible. Preferred $\mathrm{N}$ or B doping positions for different edge-functionalized AGeNRs are shown in Figure 4.1 denoted by $\mathrm{P}_{1}$ and $\mathrm{P}_{2}$. It was found that the formation energy of a single substitution at the edge site is lower than 
other sites in the nanoribbon, indicating that the possibility of substitution of Ge atoms with dopants is higher at the edges of the nanoribbons $[155,158]$. Nonetheless, other metastable sites can be considered for practical thermodynamical conditions due to the small difference of their formation energies [159].

Formation energies, band gaps, and magnetic edge states of hydrogenated and fluorinated AGeNRs doped with $\mathrm{N}$ or B atom are listed in Table 4.1. The formation energy of B-doped AGeNRs is found to be lower than that of N-doped AGeNRs, suggesting that the B impurity is easier to substitute than Ge atoms at the edge of AGeNRs. It can also be due to the larger Pauling electronegativity (3.04) and smaller covalent radius $(0.75 \AA)$ of the N impurity compared to the B impurity (Pauling electronegativity of 2.04 and covalent radius
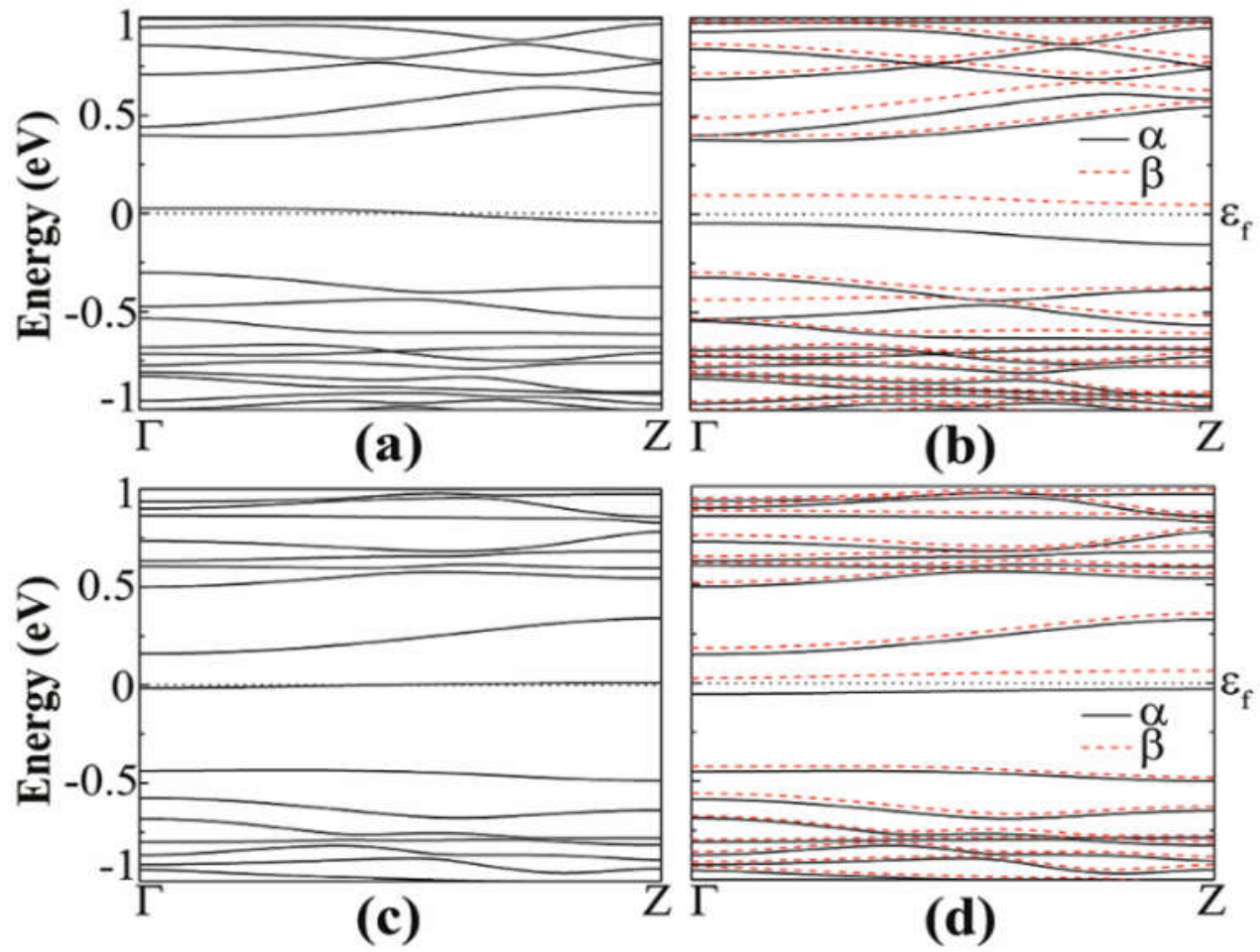

Figure 4.8 Band structure of B-doped fluorinated a11 in the (a) NM and (b) FM states, Ndoped fluorinated a11 in the (c) NM and (d) FM states. 
of $0.82 \AA)$ [156]. The optimized N-Ge (1.85 $\AA$ ) and N-H (1.02 $\AA)$ bonds for N-doping at

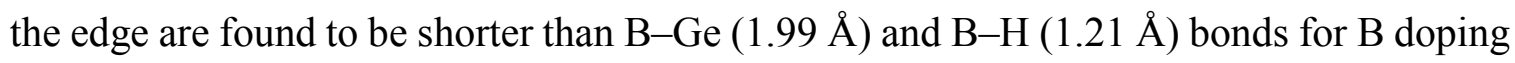
at the same edge location.

Because of the smaller ionic radius and shorter bond length of $\mathrm{N}$ doping compared to $\mathrm{B}$ doping in GeNRs, the lattice distortion of the latter is more pronounced than that of the former. It appears that B-doped AGeNRs have almost the same bond length as the pristine ones and offer a more stable structure with less lattice distortion.

The band structures of N- or B-doped AGeNRs show that there is a half-filled band near the Fermi level, resulting in a semiconductor-metal transition, as seen in Figure 4.8(b) and (d). It is interesting to find that the band introduced by an $\mathrm{N}$ dopant lies closer to the conduction band, while the band induced by a B dopant is located near valence band. The reason behind this is that the value of ionic electronegativity of the Ge atom is very close to B and lower than N. Hence the orbit energies of B are higher than those of Ge and lower for $\mathrm{N}$ than Ge. As a result, the B (N) impurity shifts the band levels of GeNRs up (down) $[160,161]$

The spin-polarized calculations of fluorinated $a_{11}$ doped with a B atom indicate that it is semiconducting in both channels with band gaps of 0.425 and $0.393 \mathrm{eV}$ for spin-up and spin-down channels, respectively, as seen in Figure 4.8(b). Figure 4.8(d) shows the spinpolarized band structure of $\mathrm{N}$-doped fluorinated $\mathrm{a}_{11}$. Interestingly enough, the valence band maximum (VBM) of the spin-up channel and the conduction band minimum (CBM) of the spin-down channel are both close to the Fermi level. An indirect band gap of $0.136 \mathrm{eV}$ was found in the spin up channel and a direct band gap of $0.439 \mathrm{eV}$ was found in spin down 
channel for the fluorinated $\mathrm{a}_{11}$ doped with $\mathrm{N}$ atom. Besides, the hydrogenated $\mathrm{a}_{11}$ doped with $\mathrm{N}$ or $\mathrm{B}$ atom is still metallic. As found from calculation, when the impurity atom (N or B) is replaced with Ge atoms at the edge, the spins on the edge are locally suppressed around the dopant atom and remain less affected on the undoped edge. As a consequence, a total magnetic moment of $0.761 \mu_{\mathrm{B}}$ (or $0.583 \mu_{\mathrm{B}}$ ) per supercell is gained for the B doped (or $\mathrm{N}$ doped).
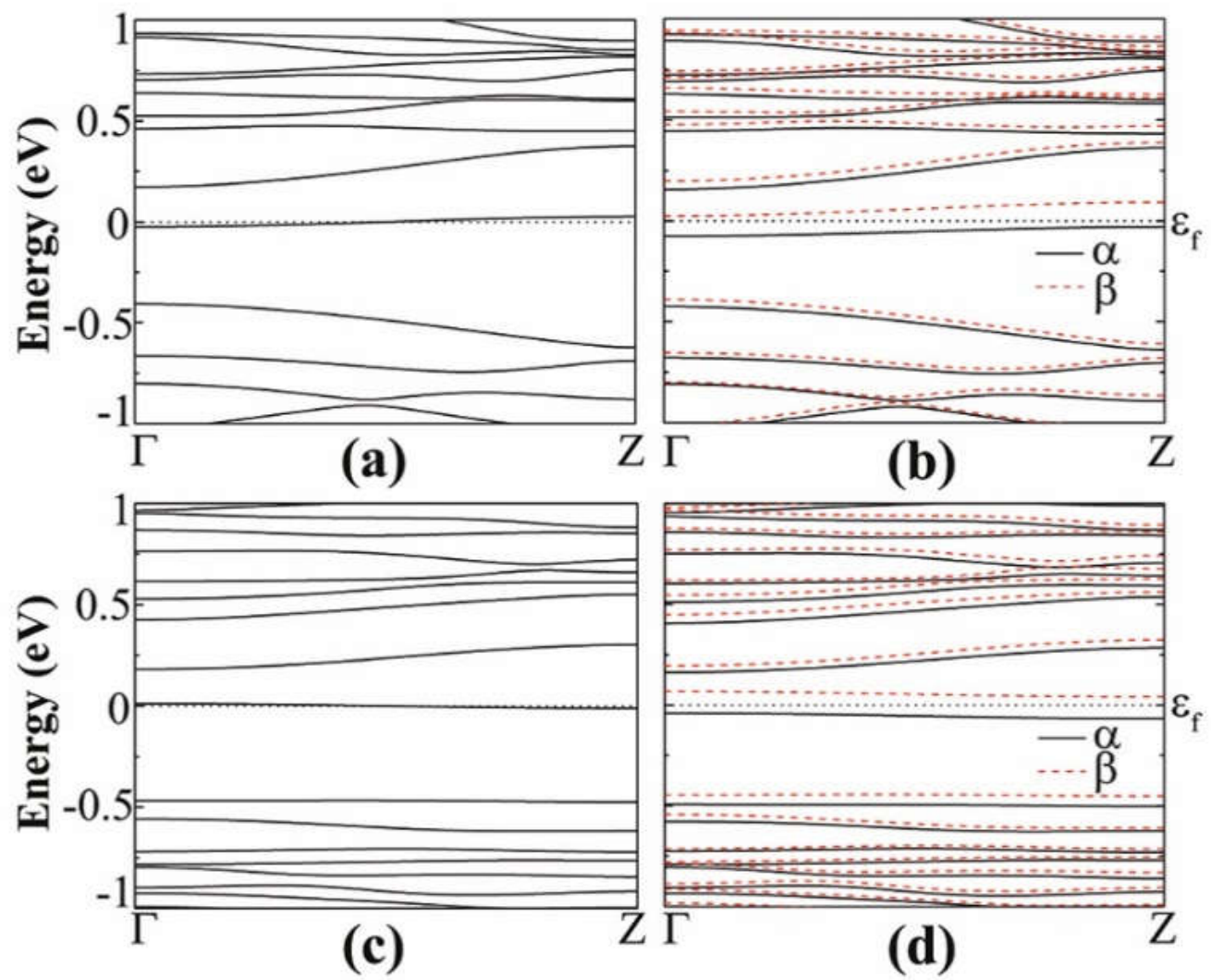

Figure 4.9 Band Structure of N-doped hydrogenated $\mathrm{a}_{11}$ in the (a) NM and (b) AFM states, N-doped fluorinated (c) NM and (d) FM states.

The spin resolved band structure of $\mathrm{N}$ - or $\mathrm{B}$-doped hydrogenated $\mathrm{z}_{11}$ shows that the introduction of dopants at the edges give rise to asymmetrical spin-up and spin-down bands 
around $\varepsilon_{\text {f, }}$ suggesting that the spin degeneracy of $\mathrm{AFM}-\mathrm{z}_{11}$ disappeared and the mirror symmetry is broken.

The spin polarized calculation of hydrogenated $\mathrm{a}_{11}$ doped with $\mathrm{N}$ next to the edge atom $\left(\mathrm{P}_{2}\right)$ of the nanoribbon shows that it is AFM semiconducting in both channels with band gaps of 0.230 and $0.414 \mathrm{eV}$ for spin-up and spin-down channels, respectively as can be seen from Figure 4.9(b). On the contrary, fluorinated $\mathrm{a}_{11}$ doped with $\mathrm{N}$ at the same position shows FM semiconducting behavior in both channels. The band gap, in this case, is 0.204 $\mathrm{eV}$, which is direct band gap in spin-up channel but indirect in spin-down channel with $0.483 \mathrm{eV}$ as shown in Figure 4.9(d). The total magnetic moment was calculated to be 0.768 $\mu_{\mathrm{B}}$ in this case.

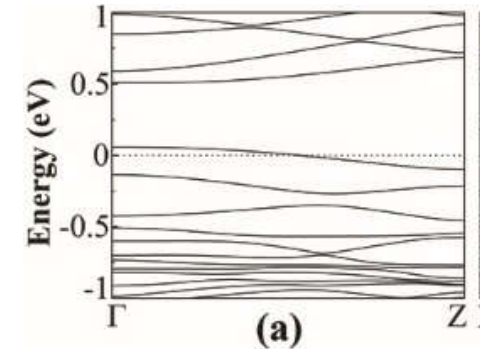

(a)

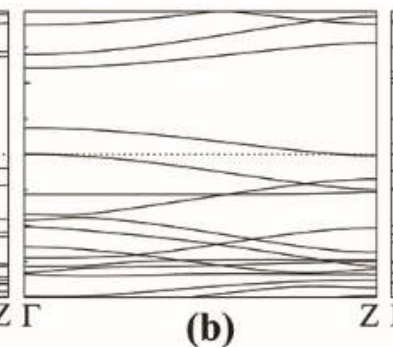

(b)

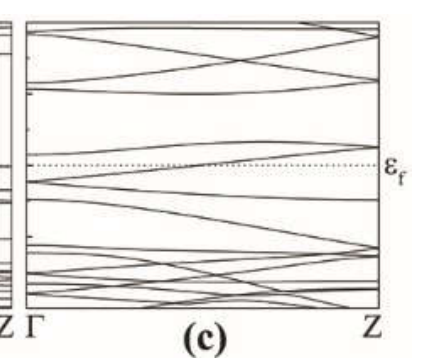

(c)

Figure 4.10 Effect of doping concentration: band structure of hydrogenated $\mathrm{a}_{11}$ when doped by (a) B, (b) 2B and (c) 3B

For higher doping concentrations, a new band appears close to valence (conduction) band for each additional boron (nitrogen) atom. Spin-polarized calculations show that hydrogenated $\mathrm{a}_{11}$ doped with double or triple boron atoms are metal, similar to the single doped case, as shown in Figure 4.10. When AGeNRs are doped by a higher concentration of nitrogen, no significant change was found except for the double nitrogen doped case. It shows semiconducting behavior with an indirect band gap of $0.241 \mathrm{eV}$. These results are similar to previous findings for silicene nanoribbons [162]. 


\subsection{Doped zigzag germanene nanoribbons}

The doping effects on ZGeNRs are considered. In this case, 7-ZGeNR is used for the supercell model containing 42 germanium atoms and 6 or 12 atoms of hydrogen or fluorine is selected. The doping position is chosen at the edge of the nanoribbon to substitute a Ge atom because of its low formation energy. This ensures the structural stability of ZGeNRs possibly due to the decay of high energy states in GeNRs, as reported for SiNRs [158]. The non-spin-polarized calculation shows that doped ZGeNRs are all metallic. Formation energies, band gaps, and magnetic edge states of hydrogenated and fluorinated ZGeNRs doped with $\mathrm{N}$ or $\mathrm{B}$ atom are listed in Table 4.3.

Table 4.3 Edge formation energy Eedge (eV), band gap Eg (eV), magnetic state, the energy difference between the FM and AFM state $\triangle \mathrm{E}_{\mathrm{FM}-\mathrm{AFM}}(\mathrm{eV})$, and spin-polarized band gap $(\mathrm{eV})$ for different $\mathrm{N}$ - and B-doped zigzag edge GeNRs with hydrogen and fluorine.

\begin{tabular}{|c|c|c|c|c|c|c|c|}
\hline \multirow{2}{*}{$\begin{array}{l}\text { Edge } \\
\text { Type }\end{array}$} & \multirow{2}{*}{$\begin{array}{l}\text { Edge } \\
\text { Atom }\end{array}$} & \multirow{2}{*}{ Dopant } & \multirow{2}{*}{$\begin{array}{c}\text { Eedge } \\
(\mathrm{eV} / \AA)\end{array}$} & \multirow{2}{*}{$\begin{array}{c}\text { Band gap } \\
(\mathrm{eV})\end{array}$} & \multirow{2}{*}{$\begin{array}{l}\text { Magnetic } \\
\text { State }\end{array}$} & \multicolumn{2}{|r|}{ Magnetic Edge State } \\
\hline & & & & & & $\Delta \mathrm{E}(\mathrm{meV})$ & Band gap (eV) \\
\hline \multirow{4}{*}{$z_{11}$} & $\mathrm{H}$ & $\mathrm{B}$ & -4.33 & 0.00 & AFM-S & & Up (0.314) (d) - Down (0) \\
\hline & $\mathrm{H}$ & $\mathrm{N}$ & -0.625 & 0.00 & AFM-S & & Up (0) (d) - Down (0.3329) (d) \\
\hline & $\mathrm{F}$ & B & -4.646 & 0.00 & AFM-S & & Up (0) (d) - Down (0.1461) (in) \\
\hline & $\mathrm{F}$ & $\mathrm{N}$ & +2.59 & 0.00 & AFM-S & & $\begin{array}{l}\text { Up (0.3081) (d) - Down (0.1318) } \\
\text { (d) }\end{array}$ \\
\hline \multirow{4}{*}{$z_{22}$} & $\mathrm{H}$ & B & -3.954 & 0.00 & AFM-S & & Up (0.3505) (d) - Down (0) (in) \\
\hline & $\mathrm{H}$ & $\mathrm{N}$ & -0.02 & 0.00 & AFM-S & & $\begin{array}{l}\text { Up (0.3379) (d) - Down (0.1189) } \\
\text { (d) }\end{array}$ \\
\hline & $\mathrm{F}$ & B & -4.62 & 0.00 & FM-S & & $\begin{array}{l}\text { Up (0.2071) (d) - Down (0.0988) } \\
\text { (d) }\end{array}$ \\
\hline & $\mathrm{F}$ & $\mathrm{N}$ & -1.05 & 0.00 & AFM-S & & 0.0 \\
\hline
\end{tabular}

$a$ : armchair, $z$ : zigzag, subscripts: the number of fictional atoms attached to Ge edge atom, NM: non-magnetic, FM: ferromagnetic, AFM: antiferromagnetic, S: semiconductor, d: direct band gap, in: indirect band gap, $\triangle \mathrm{E}$ : $\mathrm{E}_{\mathrm{FM}}-\mathrm{E}_{\mathrm{AFM}}$

For the hydrogenated B-doped case, the spin-up channel shows semiconducting behavior with a direct band gap of $0.314 \mathrm{eV}$ and the spin-down channel is still metallic respectively, as shown in Figure 4.11(b). But in the case of $\mathrm{N}$ doping, the spin-down channel is AFM 
semiconducting with a direct band gap of $0.333 \mathrm{eV}$ and the spin-up channel is metallic. The unpaired spin-up $\pi$ band and spin- down $\pi^{*}$ the band at the edge of the nanoribbons is believed to be the origin of spin-degeneracy in pristine hydrogenated $\mathrm{z}_{11}$.

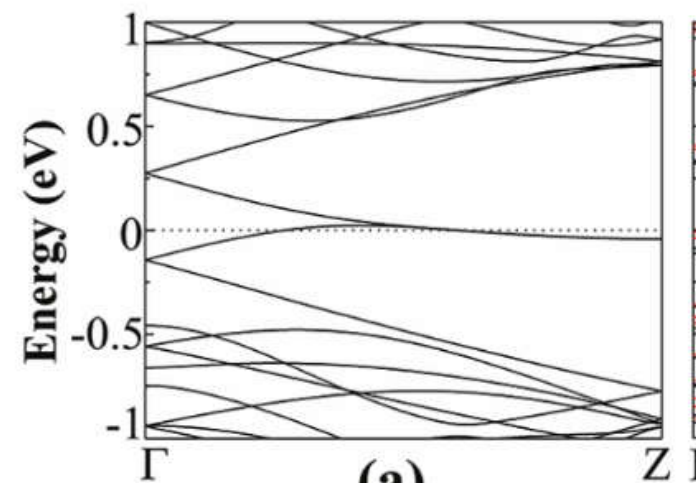

(a)
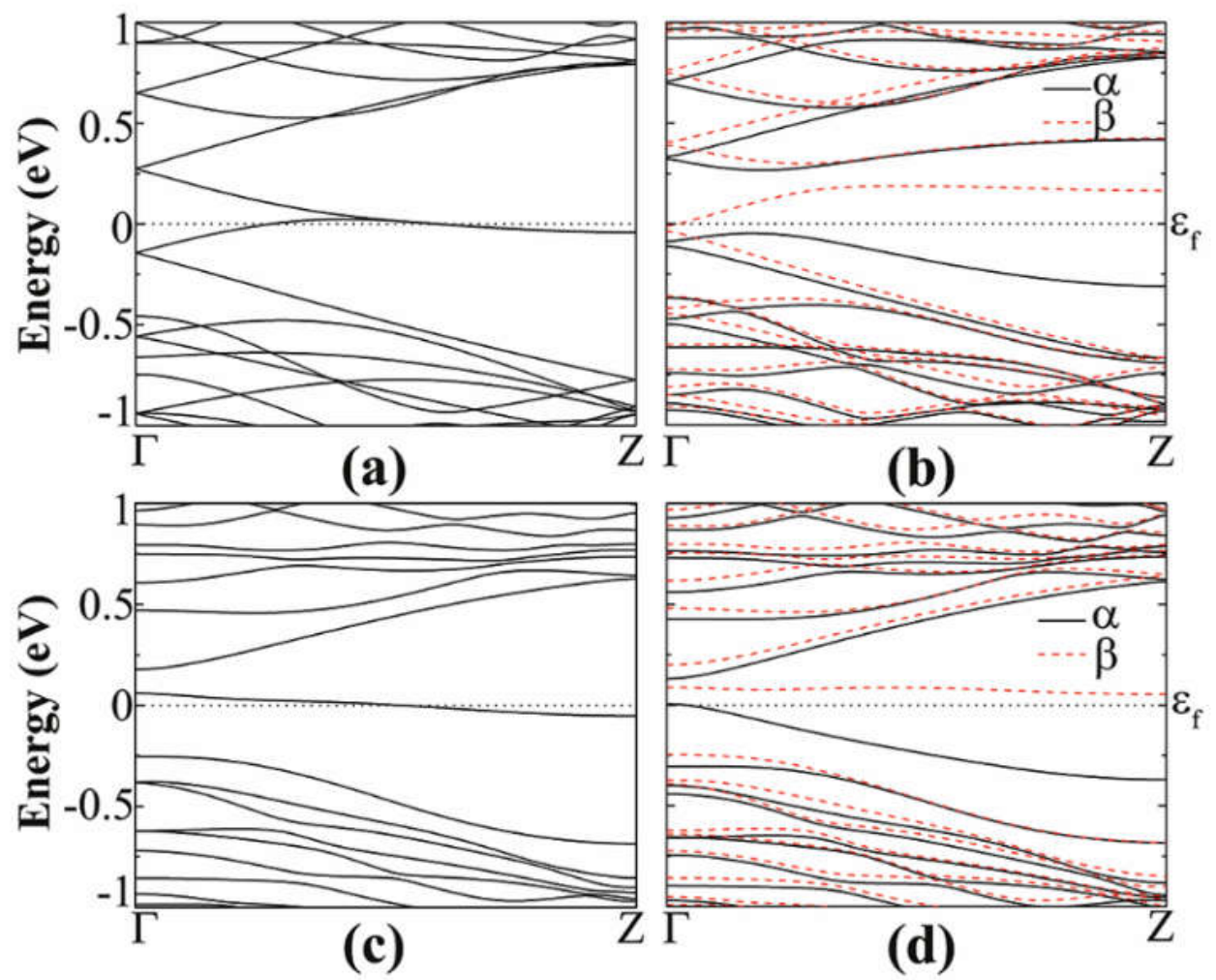

Figure 4.11 Band structure of B-doped hydrogenated $Z_{11}$ in the (a) NM and (b) AFM states and N-doped hydrogenated (c) NM and (d) AFM states.

$\mathrm{N}$ or $\mathrm{B}$ doping at the edge of the ZGeNR provides an additional electron or hole to the GeNR that can occupy the unpaired spin orbital, causing the band's degeneracy to break. Injection of extra electrons and holes into the edge of the $\pi$ and $\pi^{*}$ states more than interior states can be related to the local structural distortion caused by impurities at the edge of the nanoribbon [160, 161]. 
In the case of hydrogenated $\mathrm{z}_{22}$ when doped with $\mathrm{B}$, it transforms to a half-metallic semiconductor with a band gap of $0.351 \mathrm{eV}$ in the spin-down channel [Figure 4.12(b)] and shows total magnetic momentum of $0.907 \mu \mathrm{B}$ in the FM state. But for fluorinated B-doped, it transforms to AFM semiconductor for both of the channels with a band gap of $0.101 \mathrm{eV}$ (direct) and $0.173 \mathrm{eV}$ (indirect) in the spin-up and spin-down channel respectively [Figure 4.12(d)]. By comparing formation energies for both of the structures,
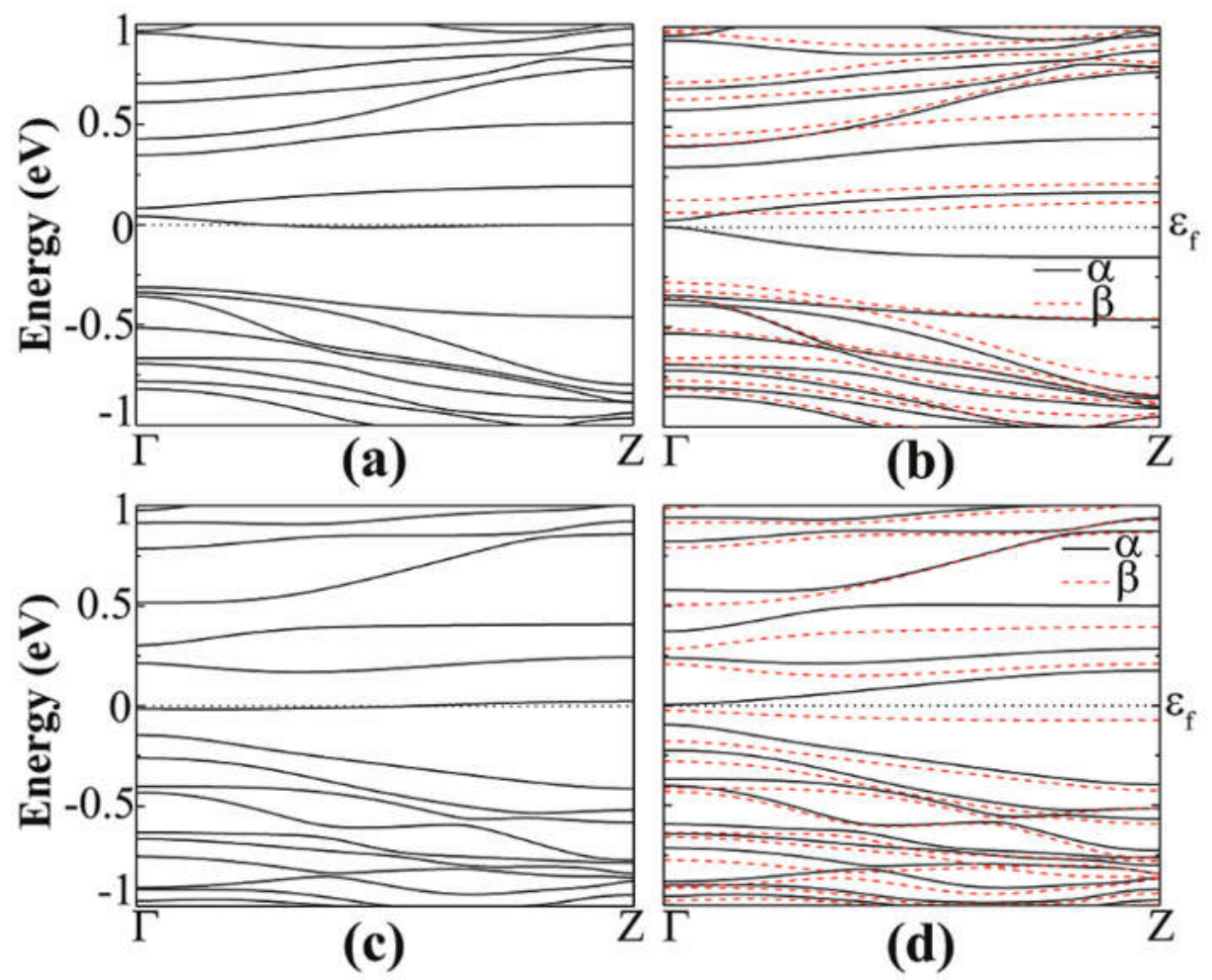

Figure 4.12 Band structure of B-doped hydrogenated $\mathrm{z}_{22}$ in the (a) NM and (b) FM states, B-doped fluorinated (c) NM and (d) AFM states.

it can be seen that the fluorinated $\left(E_{\mathrm{f}}=-4.62 \mathrm{eV}\right)$ is more stable than the hydrogenated $\left(E_{\mathrm{f}}\right.$ $=-3.95 \mathrm{eV})$. The reason can be that the Pauling electronegativity of fluorine (3.98) is 
higher than hydrogen (2.2) and also the bond length of Ge-F (1.78 $\AA$ ) is very near the GeGe bond $(2.4 \AA)[156]$.

Finally, the effect of doping concentration on electronic and magnetic properties is studied for $\mathrm{z}_{11}$. It is found that double boron and nitrogen doped ZGeNR show semiconducting behavior with very small band gaps of $0.01 \mathrm{eV}$ and $0.078 \mathrm{eV}$, respectively. Spin polarized calculations also show that triple boron/nitrogen doped $\mathrm{z}_{11}$ are NM materials.

\subsection{Conclusions}

Electronic and magnetic properties of edge functionalized (-H, -OH, -F, -Cl) AGeNRs and ZGeNRs were investigated using first principle calculations based on DFT. It was found that the halogenated structures are more stable than hydrogenated or hydroxide structures though stability decreases with increasing atomic number of halogens. Moreover, halogens still ensure more stable structures than other functionalized atoms in any case. Quantum confinement was also retained even when functionalized with other atoms rather than hydrogenated only.

This study showed that the energy band gap changes are strongly dependent on the armchair nanoribbon's width, edge atom functionalization and the number of functionalizing atoms at the edges. Edge functionalization can be used for energy band gap tuning (0.01-0.84 eV) of AGeNRs. Depending on the type of edge functionalization, the same band gap with wider ribbons can be achieved. 
Formation energy studies revealed, regardless of impurity atoms (N or B) in AGeNRs or ZGeNRs, dopants preferred to be on the edges. Single atom doped AGeNRs at one edge becomes metallic due the appearance of a half-filled impurity atom induced band which enables them to be used in negative differential resistance devices. Single atom doping (N

or B) suppresses the spin polarization of the doped edges transforming it from an AFM ground state to FM. Lastly, formation and edge free energy studies revealed the feasibility of chemical synthetization of edge functionalized germanene.

\section{CHAPTER 5}

\section{Doping and Defect Induced Germanene: A Superior Media for Sensing $\mathrm{H}_{2} \mathrm{~S}$, $\mathrm{SO}_{2}$, and $\mathrm{CO}_{2}$ gas molecules}

\subsection{Overview}

First-principles calculations based on density functional theory (DFT) have been employed to investigate the structural, electronic, and gas-sensing properties of pure, defected, and doped germanene nanosheets. Our calculations have revealed that while a pristine germanene nanosheet adsorbs $\mathrm{CO} 2$ weakly, $\mathrm{H} 2 \mathrm{~S}$ moderately, and SO2 strongly, the introduction of vacancy defects increases the sensitivity significantly which is promising for future gas-sensing applications. Mulliken population analysis imparts that an appreciable amount of charge transfer occurs between gas molecules and a germanene nanosheet which supports our results for adsorption energies of the systems. The enhancement of the interactions between gas molecules and the germanene nanosheet has been further investigated by density of states. Projected density of states provides detailed 
insight of the gas molecule's contribution in the gas-sensing system. Additionally, the influences of substituted dopant atoms such as $\mathrm{B}, \mathrm{N}$, and $\mathrm{Al}$ in the germanene nanosheet have also been considered to study the impact on its gas sensing ability. There was no significant improvement found in doped gas sensing capability of germanene over the vacancy defects, except for $\mathrm{CO} 2$ upon adsorption on $\mathrm{N}$-doped germanene.

\subsection{Introduction}

Graphene synthesis spurred the quest to obtain two-dimensional (2D) forms of other materials in group IV of the periodic table as predicted by Takeda and Shiraishi in 1994 [98]. The successful synthesis of silicene in 2012 [31] boosted the surge to obtain germanene, a 2D form of germanium analogous to graphene, bolstered by another prediction in which Cahangirov et al. found that germanene appears to be in low-buckled form [13]. Inspired by the theoretical predictions, the fully hydrogenated form of germanene, also known as germanane, was synthesized in 2013 using topochemical deintercalation of the layered calcium digermanide $\left(\mathrm{CaGe}_{2}\right)$. Dávila et al. reported experimental evidence of its synthesis by the dry epitaxial growth of germanene on a $\mathrm{Au}$ (111) surface [11]. Graphene-like germanene sheets have also been produced on Pt (111), $\mathrm{Ge} / \mathrm{Ag}(111)$, and $\mathrm{Al}(111)$ substrates and were also epitaxially and mechanically exfoliated using $\mathrm{GeH}$ synthesis on $\mathrm{SiO}_{2}[27-29,163]$.

The honeycomb structure of germanene displays ambipolar character and its charge carriers behave like massless Dirac fermions [13, 14]. Interestingly, the Fermi velocity of germanene is found to be $\sim 5.6 \times 10^{5} \mathrm{~ms}^{-1}$ and carrier interactions with phonons are 25 times less than those of graphene which may explain its high carrier mobility [164]. Once 
germanene doped or surface passivated, it offers large magnetic moment and transforms from an AFM to FM state $[99,100]$. Even with excellent electronic and magnetic properties, germanene suffers from zero energy band gap. Applying electric field [143], chemisorption of adatom species [144, 165], introducing periodic nanoholes [166], defects [167], doping [99], and edge functionalization are possible means to achieve a band gap in silicene and germanene. Previously, edge functionalization has also been used as a tool for tuning the band gap in nanoribbons [46, 50, 143-145, 168-170].

Several experimental and theoretical studies have studied graphene related materials for gas sensing [171-176]. Similar to silicene, atoms in germanene are bonded in low buckled form which is mostly instigated by the mixing of $\mathrm{sp}^{2}$ and $\mathrm{sp}^{3}$ hybridization of Ge atoms. It is predicted that germanene may have stronger adsorption of atoms and molecules than graphene due to its buckled structure [144, 177]. Xia et al. theoretically investigated common gas molecules adsorption behavior on germanene. They have found that $\mathrm{N}_{2}, \mathrm{CO}$, $\mathrm{CO}_{2}$, and $\mathrm{H}_{2} \mathrm{O}$ are physisorbed on germanene through van der Waals interactions whereas $\mathrm{NH}_{3}, \mathrm{NO}, \mathrm{NO}_{2}$ and $\mathrm{O}_{2}$ are chemisorbed through strong covalent bonds $[145,178]$. Molecular adsorption on germanene could be used either for gas sensing or band gap engineering of germanene depending on adsorption energy [145]. In our previous study, a significant improvement was found by utilizing Li-functionalized germanene as the adsorbent. Our findings suggest that $\mathrm{Li}$-functionalized germanene shows potential for $\mathrm{CO}_{2}$ capture with a storage capacity of $12.57 \mathrm{~mol} / \mathrm{kg}$ [179]. Recently, Gupta et al. forecasted the possibility of germanene in building new gas sensing material with great stability and sensitivity [180]. Padiha et al. investigated the impact of Stone-Wales and vacancies 
induced electronic and transport properties. They discovered that Stone-Wales and divacancies destroy the dispersion relation near the Fermi level to create scattering centers to reduce the current in germanene [181]. Still, germanene gas sensing capability has not been studied much compared to graphene and silicene. It is proven that defects, doping, and functionalization can certainly increase the surface-adsorbate interaction to improve the sensing activity of the materials [167]. In this study, we are going to investigate doping and defects induced gas sensing capability of germanene using density functional theory (DFT) calculations. The sensing properties of germanene nanosheet would be evaluated by considering adsorption energy, charge transfer, and density of states (DOS).

\subsection{Methodology}

First-principles DFT calculations combined with nonequilibrium Green's function (NEGF) implemented in Atomistix ToolKit (ATK) package $[130,182,183]$ are used to analyze band gap, DOS, and adsorption energy of the structures. The exchange-correlation functional uses Generalized Gradient Approximation of Perdew-Burke-Ernzerhof (PBE) to solve Kohn-Sham equations and to expand electronic density. A double- $\zeta$ polarized basis set is adopted. The Grimme vdW correction (DFT-D2) is also undertaken to describe longrange vdW interactions between germanene nanosheet and gas molecules [127, 184]. For this reason, a supplementary term $\left(\mathrm{E}_{\mathrm{vdW}}\right)$ is added to the DFT total energy ( $\left.\mathrm{E}_{\mathrm{DFT}}\right)$ to take into considerations of the vdW interactions,

$$
E_{D F T-D 2}=E_{D F T}+E_{v d W}
$$


The freestanding germanene nanosheet is modeled by $4 \times 4 \times 1$ supercell containing $32 \mathrm{Ge}$ atoms. A vacuum space of $40 \AA$ along the $\mathrm{z}$ direction in which the structures are not periodic to avoid the possible interactions between the periodically repeated unit cells. The Brillouin zone integration is sampled using Monkhorst-Pack grid of $5 \times 5 \times 1 k$-points for structural relaxations and $15 \times 15 \times 1 k$-points for total energy, electronic properties, and charge transfer calculations [185]. The density mesh cut-off is set to be 75 Hartree, and the structures are allowed to fully relax until the force on each atom becomes less than 0.01 $\mathrm{eV} / \AA \AA$

First, the adsorption energies of the incident gases on the germanene nanosheet have been evaluated using the following formula

$$
E_{a}=E_{G e-X}-E_{G e}-E_{X}
$$

where $X=\mathrm{H}_{2} \mathrm{~S}, \mathrm{SO}_{2}$, and $\mathrm{CO}_{2}$. The first, second, and third terms represent the total energies of the germanene nanosheet with adsorbed gas molecules, pristine germanene nanosheet, and the individual gas molecules, respectively. Secondly, the adsorption energies when gases adsorbed on germanene with vacancy defects and dopants have been determined by the following equation [186]

$$
E_{f}=E_{\text {defected-X/doped-X }}-E_{\text {defected/doped-Ge }}-E_{X}
$$

where $E_{\text {defected-X/doped-X }}$ and $E_{\text {defected-X/doped-X }}$ are the total energies for defected/doped germanene with gas adsorbed and without gas adsorbed in the system. Additionally, the formation energies of the vacancy defects have been calculated using the following formula 


$$
E_{f}=E_{\text {defected }}-\{(x-y) / x\} E_{\text {pristine }}
$$

where $E_{f}$ denotes the formation energy, $E_{\text {defected }}$ and $E_{\text {pristine }}$ are the total energies of the defected and pristine germanene, correspondingly, and $x$ and $y$ are the number of atoms in the germanene nanosheet and the number of atoms eliminated from the nanosheet, respectively.

\subsection{Results and Discussion}

The optimized structure of a pristine germanene sheet is shown in Figure 5.1 (a). The calculated lattice constant (4.20 $\AA)$, Ge-Ge bond length $(2.46 \AA)$, and buckling distance $(0.727 \AA)$ found in optimized geometry are in good agreement with the previous findings $[142,143,187]$. We have investigated three toxic gases $\left(\mathrm{H}_{2} \mathrm{~S}, \mathrm{SO}_{2}\right.$, and $\left.\mathrm{CO}_{2}\right)$ to bind with monolayer germanene once exposed. To start the relaxation, the gas molecules can be placed at valley, hill, bridge and hollow sites as shown in Figure 1. Gas molecules can be adsorbed at these specific sites horizontally, vertically or in a slanted form. We started with $\mathrm{H}_{2} \mathrm{~S}$ adsorption on germanene monolayer, which is one of the toxic gases produced from industrial waste and extremely lethal at concentrations $>250 \mathrm{ppm}$ [188]. The most stable configuration of $\mathrm{H}_{2} \mathrm{~S}$ molecule adsorbed on germanene is shown in Figure 5.2(a), where $\mathrm{H}_{2} \mathrm{~S}$ is aligned parallel to the surface of germanene, and the $\mathrm{S}$ atom is pointing towards the germanene monolayer at a distance of $1.45 \AA$. An adsorption energy of $-0.57 \mathrm{eV}$ is found for $\mathrm{H}_{2} \mathrm{~S}$ adsorption on pristine germanene which is better than silicene nanosheet [167] and comparable to silicene nanoribbon [189]. Full structural relaxation shows a slight elongation in the $\mathrm{H}-\mathrm{S}$ bond from 1.35 to $1.36 \AA$ and reduction in the $\mathrm{H}-\mathrm{S}-\mathrm{H}$ angle from 
91.29 to $91.12^{\circ}$. The Ge-Ge bond length varies from 2.45 to $2.49 \AA$ around the $\mathrm{H}_{2} \mathrm{~S}$ molecule while bond length remains the same (2.46 $\AA$ ) as pristine (Figure 5.1(a)) in other locations.

(a)
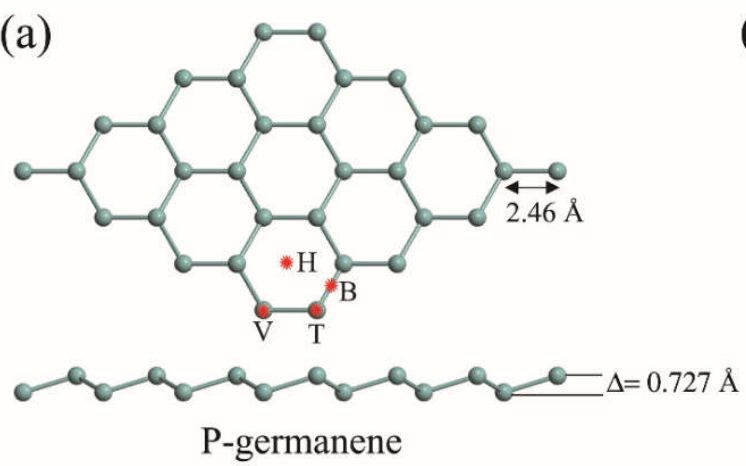

(c)

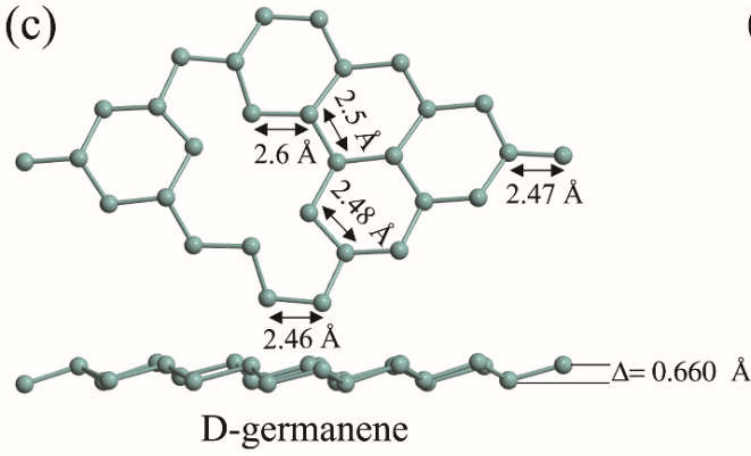

(b)
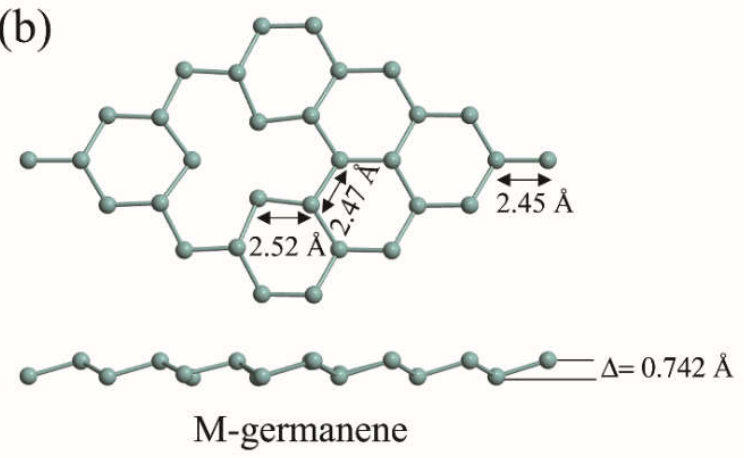

(d)

\section{M-germanene}

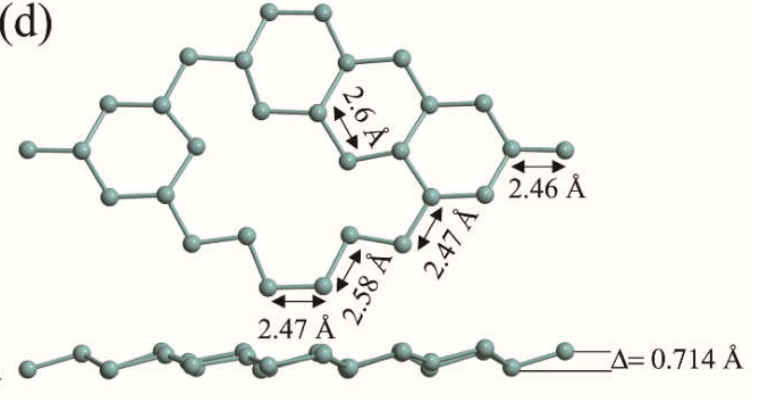

T-germanene

Figure 5.1 Top and side view of optimized structures of (a) pristine, (b) mono vacancy, (c) di vacancy, and (d) tri vacancy of germanene sheet. Possible sites for gas molecules adsorption on germanene are: T (top), V (Valley), B (bridge), and H (hollow).

Another hazardous sulfur-containing gas, $\mathrm{SO}_{2}$, the prime component of industrial waste materials, has a poisonous effect that is particularly precarious for those with respiratory as well as heart diseases [190]. The minimum energy configuration of $\mathrm{SO}_{2}$ adsorbed germanene has an adsorption energy of $-1.55 \mathrm{eV}$, and the molecule is positioned horizontally at a distance of $2.08 \AA$ from the germanene monolayer, as shown in Figure 5.2 (b). This adsorption energy is sufficiently larger than those obtained for a germanane [180], 
silicene gas sensor [167] and lower than silicene nanoribbon [189]. S-O bond length stretched to 1.58 from $1.47 \AA$ and the angle reduced to $110.2^{\circ}$ from $119.91^{\circ}$. Finally, $\mathrm{CO}_{2}$ adsorption on a germanene nanosheet was also studied. It is the most abundantly available greenhouse gas in the environment. By investigating all of the configurations, it has been found that horizontally adsorbed $\mathrm{CO}_{2}$ is the most stable configuration. This structure provides a tiny adsorption energy of $-0.05 \mathrm{eV}$ which is smaller than that of graphene [191], silicene [167] and germanene nanoribbon [145], with a relatively large germanene- $\mathrm{CO}_{2}$ distance of $3.79 \AA$ as can be seen from Figure 5.2(c). Based these findings, while $\mathrm{SO}_{2}$ is chemisorbed on pristine germanene, $\mathrm{H}_{2} \mathrm{~S}$ is physically adsorbed and $\mathrm{CO}_{2}$ offers fairly weak physisorption that limits the use of pristine monolayer germanene as a gas sensor for $\mathrm{CO}_{2}$ and $\mathrm{H}_{2} \mathrm{~S}$ detection. A possible remedy to this obstacle is to improve the adsorption by the creation of defects and introducing foreign atoms.

In this research, we focused on vacancy defects in order to enhance the sensitivity of germanene nanosheet to the toxic gases. The vacancy defects which are unavoidable in the synthesis of nanomaterials such as exfoliation of monolayers could be created by the exposure from a laser or electron beam thereby enhancing the adsorption mechanism of the adsorbate gas molecules with the host monolayers. This indispensable circumstance inspires us to form mono-, di-, and tri-vacancies in the germanene nanosheet by eliminating one, two and three germanium atoms, correspondingly. For the sake of convenience, we refer mono, di, and trivacancy germanene by M-germanene, D-germanene, and Tgermanene, respectively and the pristine one as P-germanene. The optimized atomic structures of M-germanene, D-germanene, and T-germanene are shown in Figure 5.1(b)- 
1(d). Stabilities of defects have been determined using equation 4 that are $-2.77,-3.70$ and $-5.03 \mathrm{eV}$ for mono-, di-, and tri vacancies, correspondingly which are very similar for silicene [186]. The calculated energies show that the defected structures are stable because the formation process is exothermic. It is found that the Ge-Ge bond length is elongated due to the introduction of vacancies and buckling for nanosheet changes from 0.727 to $0.742 \AA, 0.660$ and $0.714 \AA$ for mono, di, and trivacancies, respectively as seen in Figure 1.

The impact of vacancies on gas sensing mechanism has been studied by starting with Mgermanene sheet. We have considered various sites to find the most stable configuration for gas molecules to be adsorbed on M-germanene. $\mathrm{H}_{2} \mathrm{~S}$ gas molecules are strongly chemisorbed with an adsorption energy of $-2.41 \mathrm{eV}$ which is 5 times higher than its value on the pristine nanosheet. The nanosheet surface- $\mathrm{H}_{2} \mathrm{~S}$ distance considerably reduced to 1.61 $\AA$ from $2.95 \AA$ which indicates strong chemisorption and ended up in dissociation of $\mathrm{H}_{2} \mathrm{~S}$ with one of the $\mathrm{H}$ atoms dislodges itself. The other H-S bond remains in contact with a distance of $1.4 \AA$ which is larger than its previous value $(1.35 \AA)$ as an isolated molecule. The most stable configuration is presented in Figure 5.2 (d). The minimum energy structure for the case of $\mathrm{SO}_{2}$ gas adsorbed on M-germanene (in Figure 5.2 (e)) demonstrates that all of the atoms are attached to Ge atoms in the nanosheet. 

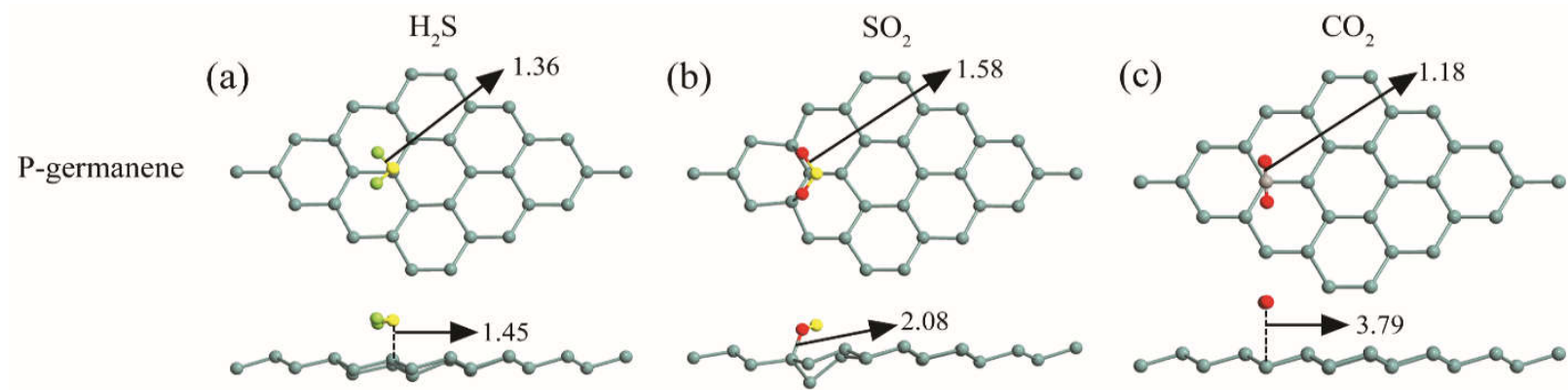

(d)

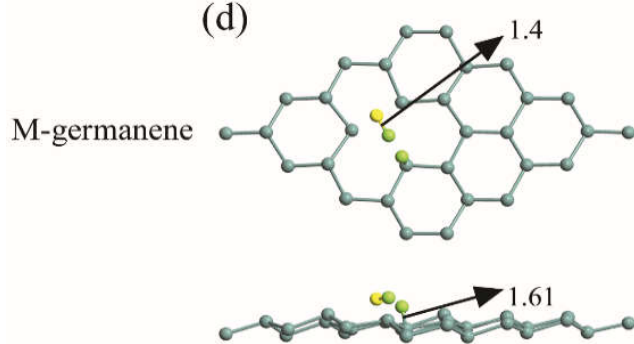

(g)

D-germanene
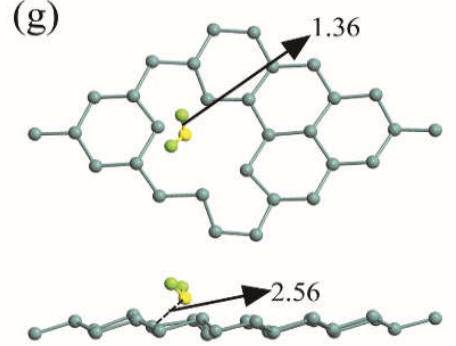

T-germanene

(j)

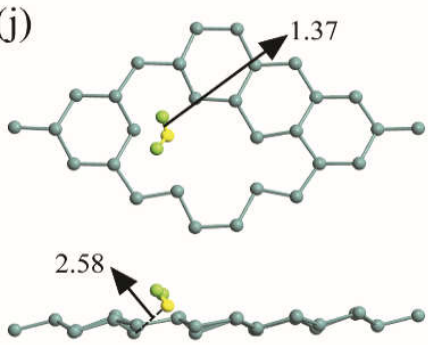

(e)
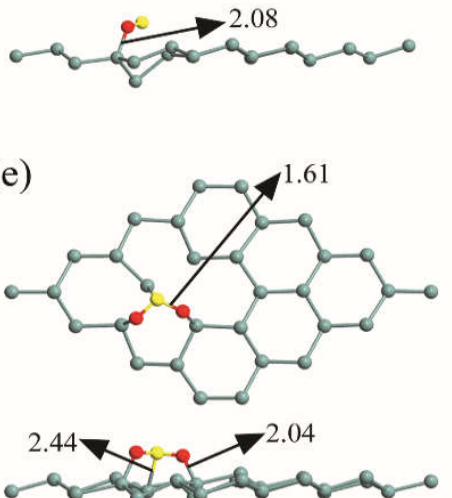

(f)
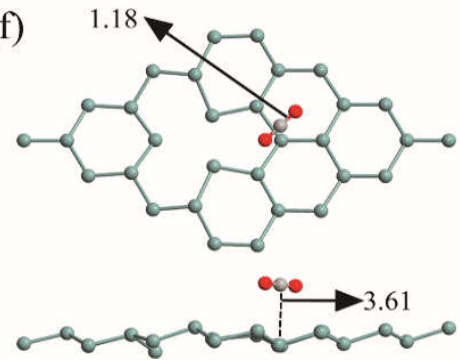

(h)
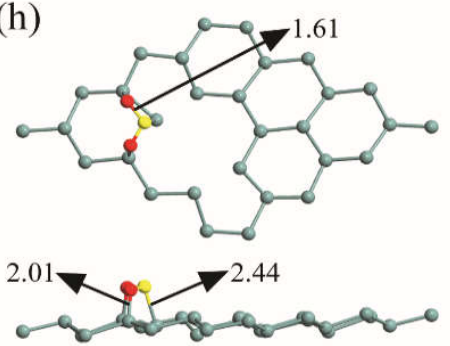

(k)

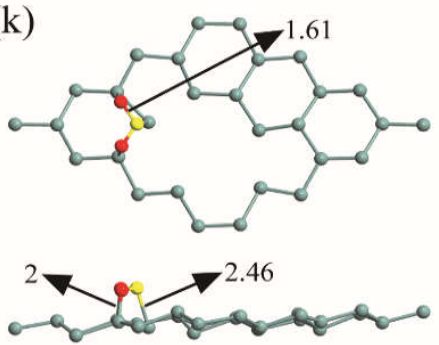

(i)
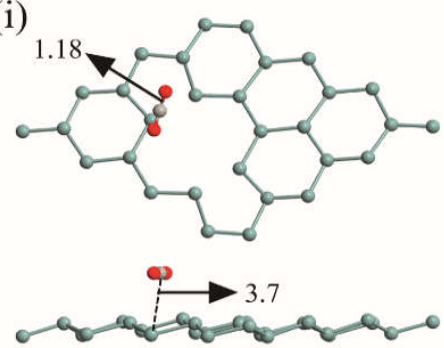

(1)

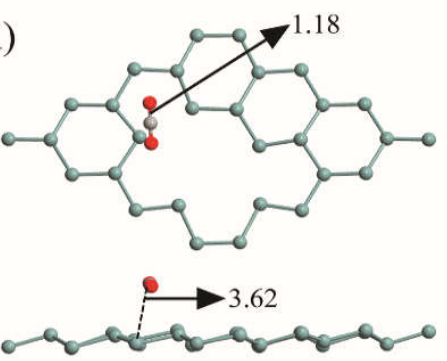

Figure 5.2 Top and side views of optimized structures of pure germanene with (a) $\mathrm{H}_{2} \mathrm{~S}$, (b) $\mathrm{SO}_{2}$, and (c) $\mathrm{CO}_{2}$, monovacancy germanene with (d) $\mathrm{H}_{2} \mathrm{~S}$, (e) $\mathrm{SO}_{2}$, and (f) $\mathrm{CO}_{2}$, divacancy germanene with (g) $\mathrm{H}_{2} \mathrm{~S}$, (h) $\mathrm{SO}_{2}$, and (i) $\mathrm{CO}_{2}$, and trivacancy germanene with (d) $\mathrm{H}_{2} \mathrm{~S}$, (e) $\mathrm{SO}_{2}$, and (f) $\mathrm{CO}_{2}$, respectively. Cyan, green, yellow, red and brown balls represent $\mathrm{Ge}, \mathrm{H}$, $\mathrm{S}, \mathrm{O}$, and $\mathrm{H}$ atoms, respectively.

A large adsorption energy of $-2.58 \mathrm{eV}$ is found in this configuration with a surface-gas distance of $2.04 \AA$. The adsorption energy $\mathrm{SO}_{2}$ is almost the same as $\mathrm{H}_{2} \mathrm{~S}$ and 1.7 times 
higher than M- germanene nanosheet. Though the bond length of S-O slightly elongated and O-S-O angle increased a little bit from 1.58 to $1.6 \AA$ and 110.2 to $111.05^{\circ}$, respectively, compared to

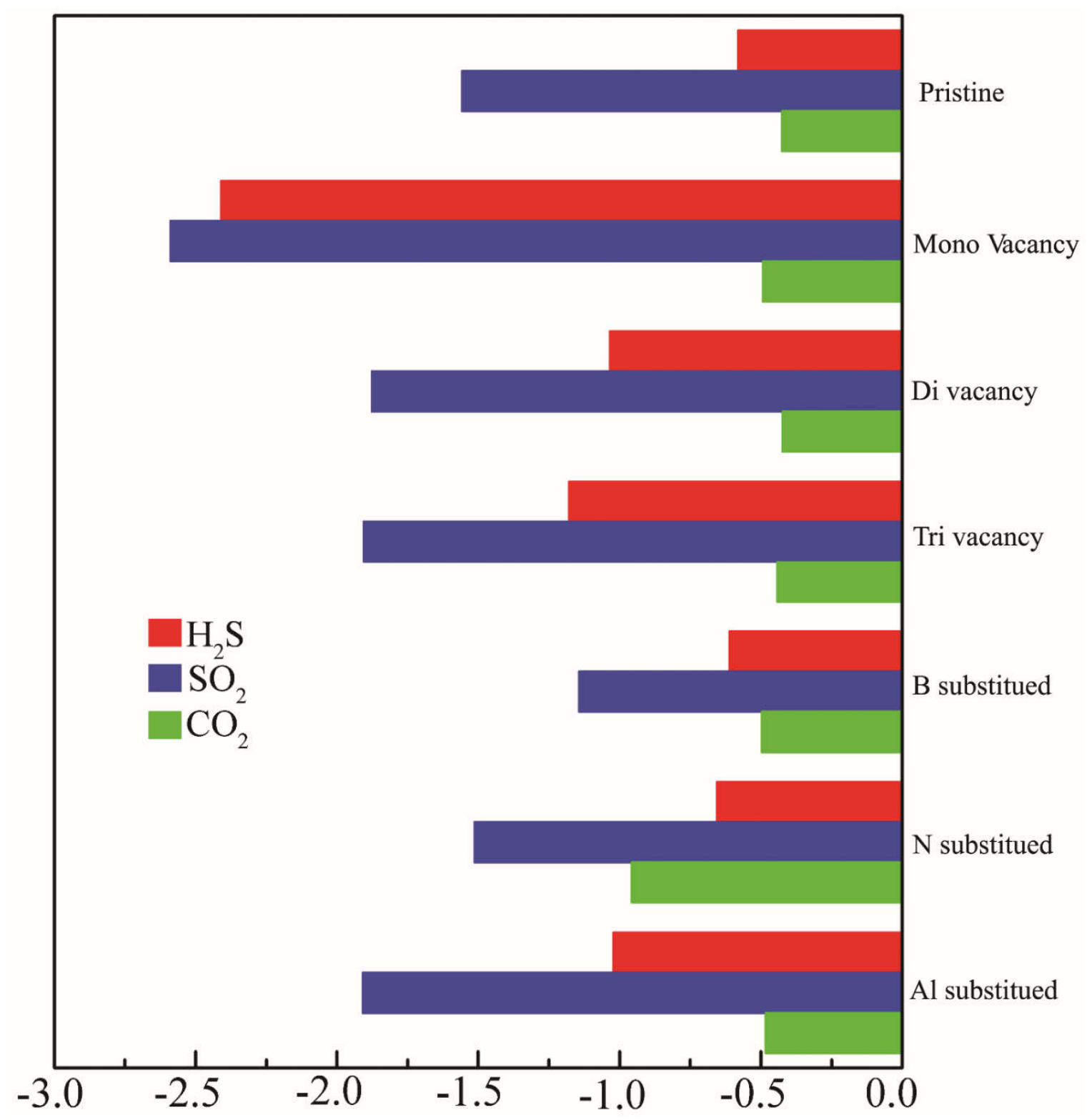

Figure 5.3 van der Waals induced adsorption energies of incident gases on pristine, defected, and substituted germanene. 
the pristine case. There is moderate improvement found for the case of $\mathrm{CO}_{2}$ adsorbance on M-germanene as in Figure 5.2 (f). It is reduced in this case which is from -0.42 to $-0.49 \mathrm{eV}$ and a binding distance of $3.61 \AA$ is calculated in this case and the bond angle remains the same as pristine.

While the addition of monovacancy in germanene nanosheet enhanced the adsorption sensitivity of the investigated gases, nonethelessthe divacancy in the system has proved to be less effective. Adsorption energies of $-1.03,-1.87$, and $-0.42 \mathrm{eV}$ have been calculated on D-germanene in the instance of $\mathrm{H}_{2} \mathrm{~S}, \mathrm{SO}_{2}$, and $\mathrm{CO}_{2}$ as shown in Figure $5.2(\mathrm{~g})$-(i), though they are still more attractive numbers compared to D-silicene [167] and GeH sensors [180]. It is observed that $\mathrm{H}_{2} \mathrm{~S}$ adsorption increases by two times while $\mathrm{SO}_{2}$ and $\mathrm{CO}_{2}$ remain the same compared to their pristine cases. The relative surface-gas distances are found to be 2.56, 2.01 and $3.7 \AA$ for $\mathrm{H}_{2} \mathrm{~S}, \mathrm{SO}_{2}$, and $\mathrm{CO}_{2}$, respectively which are higher than those of monovacancy because of the adsorption energy difference. Finally, trivacancy defects have been considered for T-germanene and the most feasible structure are shown in Figure 5.2 (j)-(1). Those structures offer adsorption energies of $-1.17,-1.9$, and $-0.44 \mathrm{eV}$ for $\mathrm{H}_{2} \mathrm{~S}, \mathrm{SO}_{2}$, and $\mathrm{CO}_{2}$, respectively. They also maintain a distance between gas and germanene atom which are of $2.58,2$, and $3.62 \AA$ for $\mathrm{H}_{2} \mathrm{~S}, \mathrm{SO}_{2}$, and $\mathrm{CO}_{2}$, respectively indicating similar adsorption behavior as D-germanene gas adsorption.

Additionally, impurity effects on the adsorption behavior of gas molecules have been investigated by replacing germanium atoms with foreign atoms in a germanene nanosheet. The dopants considered here include $\mathrm{B}, \mathrm{N}$, and $\mathrm{Al}$ atoms, which have already been replaced in the germanene sheet for diverse purposes such as tuning the electronic and magnetic 
properties $[192,193]$. All of the stable structural configurations of the doped gas sensing system have been presented in supplementary information (Figure $\mathrm{S} 1$ ). In the case of $\mathrm{H}_{2} \mathrm{~S}$ adsorption, it has been observed that B-doped and N-doped germanene do not cause significant changes in adsorption energies which are -0.61 and $-0.65 \mathrm{eV}$, respectively while

Table 5.1. Band gap and charge transfer of $\mathrm{H}_{2} \mathrm{~S}, \mathrm{SO} 2$, and $\mathrm{CO}_{2}$ gas molecules due to adsorption on germanene nanosheet.

\begin{tabular}{|c|c|c|c|c|}
\hline Gas & Structure & $\begin{array}{l}\text { Adsorption } \\
\text { Energy, eV }\end{array}$ & $\begin{array}{c}\text { Bandgap, } E_{g} \\
e V\end{array}$ & $\begin{array}{c}\text { Charge transfer, } \\
e\end{array}$ \\
\hline \multirow{7}{*}{$\mathrm{H}_{2} \mathrm{~S}$} & $\mathrm{P}-\mathrm{Ge}$ & -0.58 & 0.04 & -0.221 \\
\hline & M-Ge & -2.41 & 0.17 & 0.036 \\
\hline & D-Ge & -1.03 & 0.19 & -0.324 \\
\hline & $\mathrm{T}-\mathrm{Ge}$ & -1.18 & 0.11 & -0.329 \\
\hline & B-Ge & -0.61 & 0.27 & -0.252 \\
\hline & $\mathrm{N}-\mathrm{Ge}$ & -0.66 & 0.18 & -0.134 \\
\hline & $\mathrm{Al}-\mathrm{Ge}$ & -1.02 & 0.16 & -0.373 \\
\hline \multirow{7}{*}{$\mathrm{SO}_{2}$} & $\mathrm{P}-\mathrm{Ge}$ & -1.56 & 0.14 & 0.107 \\
\hline & M-Ge & -2.59 & 0.02 & 0.194 \\
\hline & D-Ge & -1.88 & 0.18 & 0.185 \\
\hline & T-Ge & -1.90 & 0.15 & 0.210 \\
\hline & B-Ge & -1.14 & 0.33 & -0.046 \\
\hline & $\mathrm{N}-\mathrm{Ge}$ & -1.51 & 0.31 & 0.108 \\
\hline & Al-Ge & -1.91 & 0.10 & 0.083 \\
\hline \multirow{7}{*}{$\mathrm{CO}_{2}$} & P-Ge & -0.42 & 0.00 & -0.051 \\
\hline & M-Ge & -0.49 & 0.11 & -0.054 \\
\hline & D-Ge & -0.42 & 0.25 & -0.044 \\
\hline & $\mathrm{T}-\mathrm{Ge}$ & -0.44 & 0.13 & -0.042 \\
\hline & $\mathrm{B}-\mathrm{Ge}$ & -0.50 & 0.04 & -0.038 \\
\hline & $\mathrm{N}-\mathrm{Ge}$ & -0.96 & 0.22 & 0.279 \\
\hline & Al-Ge & -0.48 & 0.06 & -0.090 \\
\hline
\end{tabular}

Al-doped germanene offers considerable improvement $(-1.2 \mathrm{eV})$. The absolute values of adsorption energy decrease upon adsorption of $\mathrm{SO}_{2}$ on B-doped $(-1.14 \mathrm{eV})$ and $\mathrm{N}$-doped 
germanene $(-1.51 \mathrm{eV})$, in which the drop in value is more pronounced in the former case. However, $\mathrm{Al}$ dopant enhances the interaction of $\mathrm{SO}_{2}$ with germanene. The adsorption energy of $\mathrm{SO}_{2}$ on $\mathrm{Al}$-doped germanene is $-1.90 \mathrm{eV}$. Although, no significant changes have found upon adsorption of $\mathrm{CO}_{2}$ on B-doped $(-0.49 \mathrm{eV})$ and $\mathrm{Al}$-doped $(-0.48 \mathrm{eV})$ germanene, nonetheless, $\mathrm{N}$-doped germanene offers a substantial improvement in adsorption of $\mathrm{CO}_{2}$. The adsorption energy of $\mathrm{CO}_{2}$ on $\mathrm{N}$-doped germanene $(-0.95 \mathrm{eV})$ is almost two times greater than that of pristine germanene. Adsorption energies calculated for defected and doped germanene-gas sensing system are presented in Figure 5.3 for comparison. An electronic charge transfer occurs when gas molecules adsorb on germanene nanosheet. The amount of charge transfer between surface and gas molecules have been calculated using Mulliken population analysis [194] and is summarized in Table 5.1 for the most stable configurations considered here. The positive value of charge transfer indicates gas molecules gain charges from the surface while negative values means the surface receives charges from gases. $\mathrm{H}_{2} \mathrm{~S}$ and $\mathrm{CO}_{2}$ gas molecules are electron-donating gas molecules whereas $\mathrm{SO}_{2}$ has the electron extracting capability. From the Table 5.1, it can be seen that $\mathrm{H}_{2} \mathrm{~S}$ donates a significant amount of charge to the D-Ge (-0.324e), T-Ge (-0.329e), B-Ge $(-0.252 e)$ and $\mathrm{Al}-\mathrm{Ge}(-0.373 e)$ system. $\mathrm{H}_{2} \mathrm{~S}$ molecules show strong adsorption in monovacancy defected germanene where a tiny amount of charge transfer occurs due to one of the $\mathrm{H}$ atoms dislodging itself from $\mathrm{H}-\mathrm{S}-\mathrm{H}$ bond and adsorbed strongly to make a bond with the germanium atom on the nanosheet, as shown in Figure 2(d). Hence, dissociated $\mathrm{H}$ atom gains charges of $0.099 e$ due to the higher electronegativity of hydrogen atom than germanium and deserted H-S bond loses charge in this process. 
Being an electron donating character, $\mathrm{CO}_{2}$ adsorption on germanene nanosheet shows similar charge transfer characteristics as $\mathrm{H}_{2} \mathrm{~S}$. Compared to pristine germanene, it has been seen that vacancy defects and doping do not change much in the adsorption energy or the charge transfer of $\mathrm{CO}_{2}$ except in the case of $\mathrm{N}$-doped germanene, where a strong adsorption energy of $-0.96 \mathrm{eV}$ achieved with a large charge transfer of $0.279 e$. The most stable configurations for each case are shown in Figure S1. Though the charge transfer is expected to be negative, the gas molecule being adsorbed on nitrogen gains charges because of higher electronegativity of nitrogen compared to germanium. Also, one of the oxygen atoms of $\mathrm{CO}_{2}$ makes a bond with a germanium atom on the surface to support the idea of charge gain on it. The same occurrence happens again because of C-Ge and O-Ge bond creation on the $\mathrm{M}-\mathrm{Ge}$ nanosheet upon $\mathrm{CO}_{2}$ adsorption, as shown in Figure 2(f). This process transfers $0.191 e$ charge to the gas molecule as carbon and oxygen are more electronegative compared to germanium atoms on the surface. Finally, $\mathrm{SO}_{2}$ molecules transfer charges to the germanene surface upon adsorption which are $0.107 e, 0.194 e, 0.185 e, 0.210 e$, and 0.108e for P-Ge, M-Ge, D-Ge, T-Ge and N-Ge systems respectively.

The origin of physisorption and chemisorption can be understood further using the density of states (DOS) analysis of surface-adsorbate systems. Figure 5.4 presents the DOS of the pristine germanene-gas systems. It can be seen that the highest occupied molecular orbital (HOMO) and the lowest unoccupied molecular orbital (LUMO) of $\mathrm{H}_{2} \mathrm{~S}$ and $\mathrm{CO}_{2}$ are located away from the Dirac point (Fermi level). Therefore, the Dirac cone properties of germanene remain almost unaffected when $\mathrm{CO}_{2}$ and $\mathrm{H}_{2} \mathrm{~S}$ molecules are adsorbed. Therefore, they are weakly physisorbed on germanene, approaching the case of $\mathrm{H}_{2} \mathrm{~S}$ and $\mathrm{CO}_{2}$ adsorbed on 
silicene [167]. However, the changes are more pronounced for $\mathrm{H}_{2} \mathrm{~S}$ adsorption. Quite the reverse, $\mathrm{SO}_{2}$ gas molecule adsorption on pristine germanene nanosheet greatly influence the electronic properties. The resulting structural deformation induces symmetry breaking vanishing the Dirac cone completely through strong Ge-O bond illuminating that the $\mathrm{SO}_{2}$ is strongly chemisorbed. As previously studied that the atoms in the germanene layer are offset from the atomic plane, the typically perpendicular $\mathrm{p}_{z}$ orbital is gradually interacting with the $\mathrm{sp}^{2}$ orbitals due to the reduced angle between them resulting in a mixed-phase material that is predominantly sp ${ }^{2}$ with a small amount of $\mathrm{sp}^{3}$ character present [164]. This phenomenon makes germanene more reactive than graphene to the gas molecules.
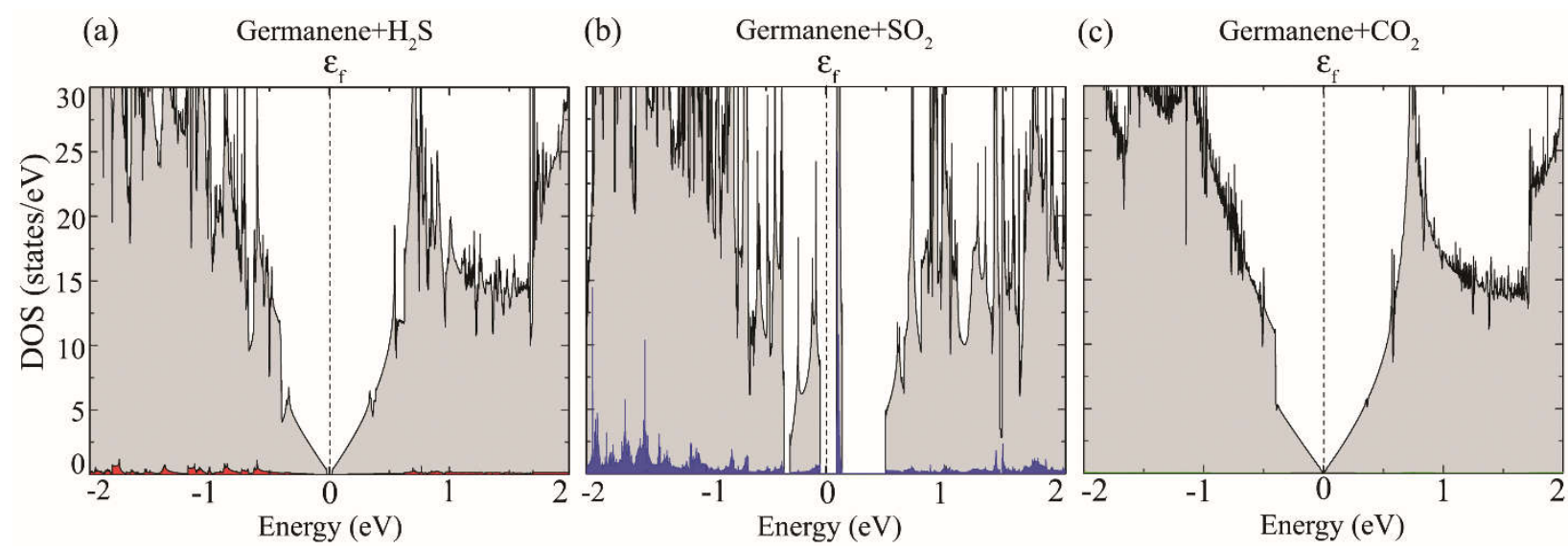

Figure 5.4 The density of states (DOS) of the pristine germanene nanosheet adsorbed by (a) $\mathrm{H}_{2} \mathrm{~S}$ and (b) $\mathrm{SO}_{2}$ and (c) $\mathrm{CO}_{2}$ molecule. The grey shaded area represents the total DOS, while the red, blue, and green colors account for the projection of DOS onto $\mathrm{H}_{2} \mathrm{~S}, \mathrm{SO}_{2}$, and $\mathrm{CO}_{2}$ molecule, respectively. Here the Fermi level is shifted to zero.

Additionally, it can be seen that some states are near the Fermi level indicating p-type doping of germanene by $\mathrm{SO}_{2}$ adsorption on pristine germanene sheet, which agrees well with the positive charge transfer on $\mathrm{SO}_{2}$ upon interaction with pristine germanene. Due to 
strong chemisorption of $\mathrm{SO}_{2}$ on germanene sheets make them a promising candidate for future $\mathrm{SO}_{2}$ gas sensing device.
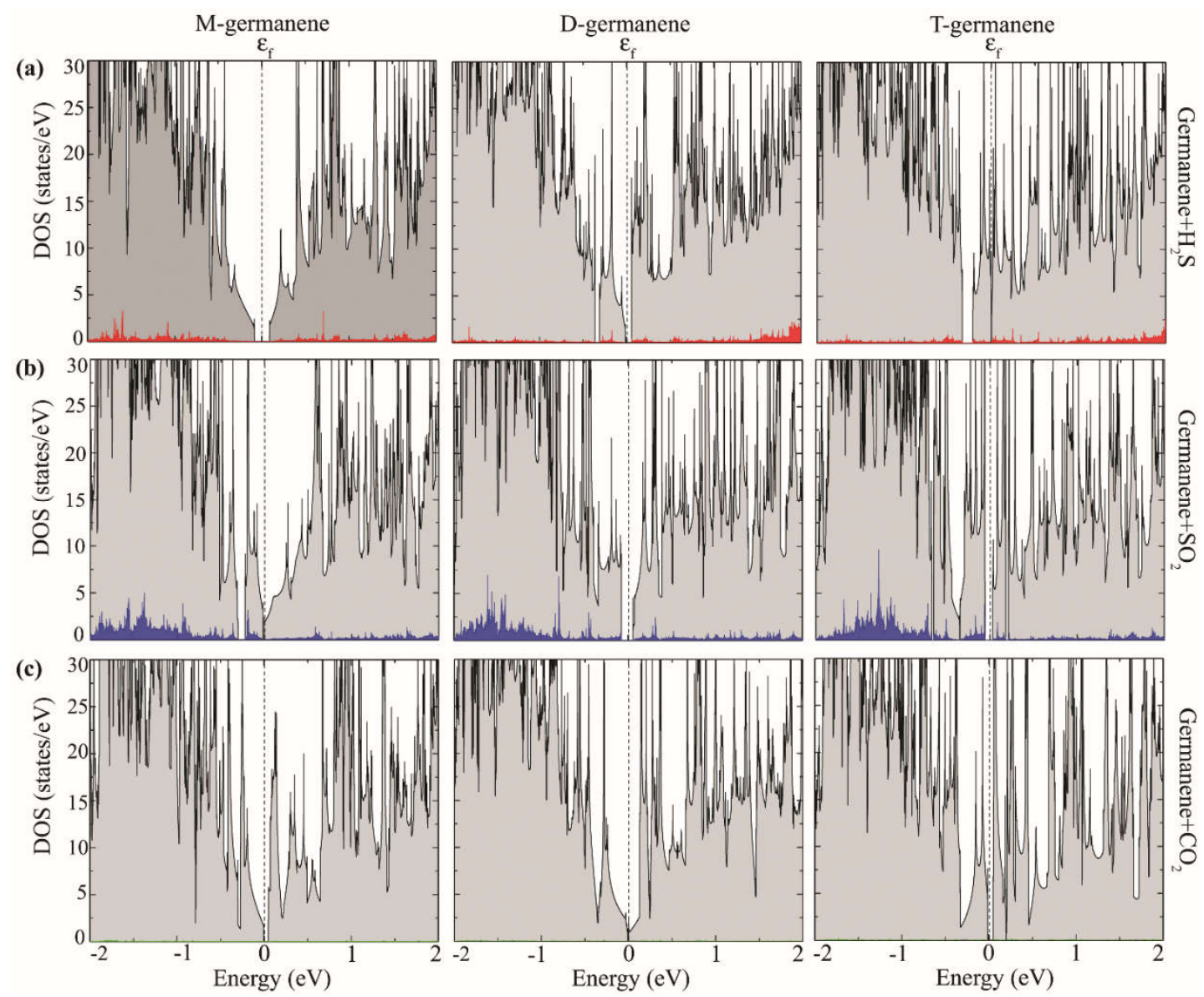

Figure 5.5 The density of states (DOS) of the vacancy defected germanene nanosheet adsorbed by (a) $\mathrm{H}_{2} \mathrm{~S}$ and (b) $\mathrm{SO}_{2}$ and (c) $\mathrm{CO}_{2}$ molecule. The grey shaded area represents the total DOS, while the red, blue, and green colors account for the projection of DOS onto $\mathrm{H}_{2} \mathrm{~S}, \mathrm{SO}_{2}$, and $\mathrm{CO}_{2}$ molecule, respectively. Here the Fermi level is shifted to zero.

DOS can provide detail insight in response to higher adsorption energies enhanced by vacancy defects in germanene as shown in Figure 5.5. Dissimilar to P-germanene, adsorption of gas molecules on M-germanene leads to a significant influence in the DOS because of strong S-Ge, $\mathrm{C}-\mathrm{Ge}$, and $\mathrm{Ge}-\mathrm{H}$ bonds in $\mathrm{SO}_{2}, \mathrm{CO}_{2}$, and $\mathrm{H}_{2} \mathrm{~S}$, respectively. The bond strength is believed to be enhanced due to the intensive hybridization of C-Ge and S- 
Ge bonds. Moreover, M-germanene turns to semiconducting upon gas molecules adsorption and single point vacancy defect results in localized states to cross bands linearly at $K$-point to open the band gap due to symmetry breaking [195].

In the meantime, D-germanene exhibits reduced adsorption energies upon gas molecules adsorption which could be due to less reactivity of germanene atoms in this structure compared to M-germanene. Still, there is no formation of the ring-like structure as in Dsilicene in the Ref. [167], and this is why D-germanene exhibits strong adsorption for $\mathrm{H}_{2} \mathrm{~S}$ and $\mathrm{SO}_{2}$ as compared to P-germanene. From the DOS analysis in Figure 5.5 (c), it is easily discernible that $\mathrm{CO}_{2}$ shows a smaller impact on the total DOS of germanene- $\mathrm{CO}_{2}$ system. On the contrary, $\mathrm{H}_{2} \mathrm{~S}$ located at the position of the removed Ge atoms provide significant hybridization of $\mathrm{S}-\mathrm{Ge}$ and $\mathrm{H}-\mathrm{Ge}$ near the Fermi level. Interestingly, $\mathrm{SO}_{2}$ forms a strong bond through Ge-O to provide strong adsorption similar to P-germanene. Finally, trivacancy does not show any significant improvement in adsorption energies over Dgermanene for $\mathrm{H}_{2} \mathrm{~S}, \mathrm{SO}_{2}$, and $\mathrm{CO}_{2}$.

In summary, it is worth mentioning that $\mathrm{P}$-germanene is reasonably sensitive to $\mathrm{H}_{2} \mathrm{~S}$ while $\mathrm{SO}_{2}$ shows strong adsorption, and $\mathrm{CO}_{2}$ has comparatively weak adsorption. The recovery time of the gas sensing material is the parameter to evaluate the performance of a gas sensor [176]. The recovery time $\tau$ can be calculated using the conventional transition state theory describe by the following formula,

$$
\tau \propto v_{0}^{-1} \exp \left(-E_{a d} / \mathrm{k}_{\mathrm{B}} \mathrm{T}\right)
$$


Here, $v \square$ is the attempt frequency. The relation established by the equation shows that the larger the adsorption energy (more negative) the longer the recovery time required for the particular sensor. Adsorption energies for recoverable gas sensing device are moderate because of reasonable recovery time in the process of desorption. Meanwhile, the defected germanene make it useful for disposable gas sensing devices due to strong adsorption energies. Although $\mathrm{CO}_{2}$ is weakly adsorbed on defected germanene with almost same adsorption energy as P-germanene, it shows moderate adsorption on N-doped germanene, making it suitable for reusable sensing material.

\subsection{Conclusion}

It was found that $\mathrm{CO}_{2}$ gas molecules shows weak adsorption while $\mathrm{H}_{2} \mathrm{~S}$ is moderate and $\mathrm{SO}_{2}$ shows strong adsorption on pristine germanene nanosheet. It has also been investigated that defects and doping in germanene change the respective electronic properties and the adsorption characteristics significantly. M-silicene shows a dramatic increase in adsorption energies of $\mathrm{H}_{2} \mathrm{~S}$ and $\mathrm{SO}_{2}$ which are 4 times and 1.66 times higher compared to pristine germanene nanosheet. Increasing defects does not improve the adsorption over Mgermanene while T-germanene is the least effective case. Strong chemisorption is observed in the case of $\mathrm{SO}_{2}$ with vacancy defect or doping in a germanene nanosheet. The electronic properties portrayed by the density of states of the germanene nanosheet system with different defects and substitutions are changed as a consequence of the charge-transfer mechanism between germanene-gas molecules. The doping of germanene with $\mathrm{B}, \mathrm{N}$, and

$\mathrm{Al}$ dopants does not seem to alter the adsorption energy for $\mathrm{H}_{2} \mathrm{~S}$ and $\mathrm{SO}_{2}$, but for $\mathrm{N}$-doped 
germanene, the adsorption of $\mathrm{CO}_{2}$ increases a lot. Finally, it could be concluded that defects and doping in germanene increase the adsorption with a cost of sensing devices to be irreversible. 


\section{CHAPTER 6}

\section{Band Gap Opening and Optical Absorption Enhancement in Graphene using ZnO Nanoclusters}

\subsection{Overview}

Electronic, optical and transport properties of the graphene/ $\mathrm{ZnO}$ heterostructure have been explored using first-principles density functional theory. The results show that $\mathrm{Zn}_{12} \mathrm{O}_{12}$ can open a band gap of $14.5 \mathrm{meV}$ in graphene, increase its optical absorption by 1.67 times covering visible spectrum which extended to infra-red (IR) range, and create slight nonlinear $I-V$ characteristics depending on the applied bias. These findings envisage that graphene $/ \mathrm{Zn}_{12} \mathrm{O}_{12}$ heterostructure can be appropriate for energy harvesting, photodetection, and photochemical devices.

\subsection{Introduction}

Graphene as a 2D carbon allotrope has drawn tremendous attention worldwide because of its unique properties for electronics, spintronics, and surface sciences $[1,6]$. It is a promising material for high-performance nanoelectronics due to its high carrier concentration, mobility, and stability [86, 87]. However, widespread adoption of graphene

for electronic devices in particular still faces challenges because it lacks an energy band gap as a result of band degeneracy at the Dirac point. Breaking translational symmetry or sublattice symmetry could be an option to introduce a band gap in graphene. From density functional studies, it is apparent that a band gap could be opened in zero band gap 2D nanomaterials in variety of ways including chemical edge-functionalization $[46,47]$ and quantum confinement in nanoribbons [37, 46, 47, 90,93], nanomesh [196], periodic 
nanoholes [93, 94], adsorption of gas molecules [179], silicon doping [197], strain and vacancy induced $[198,199]$ etc. Experimentally it has been proven that a band gap ranging from $2.5 \mathrm{meV}$ to $450 \mathrm{meV}$ can be opened in graphene with the application of strain or electric-field [200], decoration with Si-rich two-dimensional islands [88] and the introduction of defects have been used in efforts to induce a band gap [94], formation of perforated graphene using black copolymer (BCP) lithography [95], adsorption of patterned hydrogen [96] and water molecules [97]. Most of these methods require precision tools or complex lithographic process to achieve band gap even though it could be negligible sometimes. Pala et al. investigated a novel process to open band gap by decorating graphene using different nanoparticles where special attention was given to $\mathrm{ZnO}$ nanoseeds [92].

$\mathrm{ZnO}$ has a wide band gap of $3.37 \mathrm{eV}$ and large exciton binding energy of $\sim 60 \mathrm{meV}(2.4$ times of the room-temperature thermal energy) [91]. Hence, the incorporation of $\mathrm{ZnO}$ with graphene could be a promising heterostructure for electronic and optoelectronic devices such as solar cells, field emission, displays, sensors, light emitting, and detection devices in UV-visible spectral range. It could also be used in the photocatalytic degradation of organic pollutants under UV or visible light irradiation [201, 202]. ZnO nanoparticles also possess thermal and mechanical stability and require a rapid and inexpensive synthesis technique using available cost-effective standard semiconductor device fabrication technologies [91].

The experimental absorption energies of small $\mathrm{ZnO}$ nanoparticles are found to be very 
close to the optical excitation energy of $\mathrm{Zn}_{12} \mathrm{O}_{12}$ cluster calculated in the framework of time-dependent density functional theory (DFT) [203]. This observation leads the authors of Ref. [203] to conjecture that the surface structure of small $\mathrm{ZnO}$ nanoparticles is very similar to that of $\mathrm{Zn}_{12} \mathrm{O}_{12}$ nanocluster because the excitation energies of former $(\sim 3.83 \mathrm{eV})$ are in good agreement with that of later $(3.83 \mathrm{eV})$. Therefore, we have used $\mathrm{Zn}_{12} \mathrm{O}_{12}$ nanocluster to imitate nanoseeds on graphene.

In the present work, first-principles calculations are employed to investigate the effects of $\mathrm{Zn}_{12} \mathrm{O}_{12}$ on the electronic, transport, and optical properties of graphene. We found that the current-voltage response of graphene/ $\mathrm{Zn}_{12} \mathrm{O}_{12}$ heterostructure is slightly non-linear due to the occurrence of a small band gap in graphene. In addition, the optical absorption coefficient of the heterostructure reflects enhanced optical properties.

\subsection{Computational Methodology}

Calculations are performed using first-principle methods based on DFT implemented in Atomistix ToolKit (ATK) package [148]. The exchange-correlation functional is approximated by the Generalized Gradient Approximation of Perdew-Burke-Ernzerhof (GGA-PBE) with a double- $\zeta$ polarized basis set $[121,130,149]$. First, $\mathrm{Zn}_{12} \mathrm{O}_{12}$ nanocluster is optimized as shown in Fig. 6.1 (a) using GGA-PBE method. Then, the graphene/ $\mathrm{Zn}_{12} \mathrm{O}_{12}$ heterostructure, consisting of $5 \times 5$ supercell of graphene and a $\mathrm{Zn}_{12} \mathrm{O}_{12}$ nanocluster (as shown in Figure 6.2 (a) and (b)), has been fully relaxed (lattice constant and atomic positions) until the maximum atomic force and stress on each atom are less than $0.01 \mathrm{eV} / \AA$, $0.05 \mathrm{eV} / \AA^{3}$, respectively. A grid mesh cut-off energy of $75 \mathrm{Ha}$ and $1 \times 3 \times 3 \mathrm{k}$-points for the 
integration of the first Brillouin zone are chosen. A vacuum separation of $15 \AA$ perpendicular to the structure is considered to form a free-standing ultrathin film to suppress adjacent images interactions. To describe long-range van der Waals (vdW) interactions, the Grimme vdW correction (DFT-D2) is considered [127]. The k-points are increased to $1 \times 101 \times 101$ for calculations of electronic, transport, optical properties.

The electron transport calculations are performed using GGA/PBE combined with nonequilibrium green's function (NEGF). A fast Fourier transform (FFT) solver is used to solve Poisson equation.

The schematic of two electrode system made of Graphene $/ \mathrm{Zn}_{12} \mathrm{O}_{12}$ heterostructure presented in Figure 6.2(c). The electrical current through the device under non-equilibrium conditions, i.e. for a finite bias voltage ( $V_{\text {Bias }}$ ), can be calculated using the Landauer formula as follows [204]

$$
I\left(V_{\text {Bias }}\right)=\frac{2 e}{h} \int_{-\infty}^{+\infty} T\left(\varepsilon, V_{\text {Bias }}\right)\left[f\left(\varepsilon-\mu_{\mathrm{L}}\right)-f\left(\varepsilon-\mu_{\mathrm{R}}\right)\right] \mathrm{d} \varepsilon
$$

Where $f$ is the Fermi-Dirac distribution function, $\mu_{\mathrm{L}, \mathrm{R}}=E_{\mathrm{F}} \pm e V_{\mathrm{Bias}} / 2$ represent the chemical potentials of the left and right electrodes, and $T\left(\varepsilon, V_{\text {Bias }}\right)$ is the energy and voltage dependent transmission function. The transport setup consists of two graphene electrodes of $2 \AA$ and graphene/ $\mathrm{Zn}_{12} \mathrm{O}_{12}$ structure as central region. The supercell was oriented such way that transport direction connecting $\mathrm{L}$ and $\mathrm{R}$ was aligned parallel to the armchair direction within the structure, as shown in Figure 6.2 (c).The optical absorption spectrum is calculated using the Kubo-Greenwood formula [205]. The susceptibility tensor in this method given as follows 


$$
\chi_{i j}(\omega)=-\frac{e^{2} \hbar^{4}}{m^{2} \varepsilon_{0} V \omega^{2}} \sum_{n m} \frac{f\left(E_{m}\right)-f\left(E_{n}\right)}{E_{n m}-\hbar \omega-i \hbar \Gamma} \times \pi_{m m}^{i} \pi_{m n}^{j}
$$

Where, $\pi_{n m}^{i}$ means $i$-component of the dipole matrix element between $n$ and $m$ states, $\Gamma$ is the broadening, $V$ is the volume, $f$ is the Fermi function and $E_{m}\left(E_{n}\right)$ corresponds to eigenvalues of $m(n)$ state. The dielectric constant $\left(\varepsilon_{r}\right)$ can be written in terms of the susceptibility tensor $\chi(\omega)$, using linear response theory as follows

$$
\varepsilon_{r}(\omega)=1+\chi(\omega)
$$

The refractive index, $n$, is related to the complex dielectric constant through

$$
n+i \kappa=\sqrt{\varepsilon_{r}}
$$

Here, $\kappa$ is the extinction coefficient. Finally, the optical absorption coefficient is related to the extinction coefficient through [206]

$$
\alpha_{a}=2 \frac{\omega}{C} \kappa
$$

For optical properties calculation, the Hartwigsen, Goedecker, Hutter (HGH) pseudopotentials used with a good description of virtual states far above the Fermi level.

\subsection{Results and Discussion}

In the $\mathrm{Zn}_{12} \mathrm{O}_{12}$ nanocluster, the optimized length of $\mathrm{Zn}-\mathrm{O}$ bonds are $1.85 \AA$ which is shared between two hexagon rings and $2.02 \AA$ which is shared between a tetragon and hexagon ring (Fig. 6.1(a)). From the molecular orbital energy levels as shown in Fig. 6.1(b), it is seen the $\mathrm{Zn}_{12} \mathrm{O}_{12}$ nanocluster is a semiconductor with the HOMO-LUMO (highest occupied molecular orbital- lowest unoccupied molecular orbital) energy gap of $2.43 \mathrm{eV}$. 


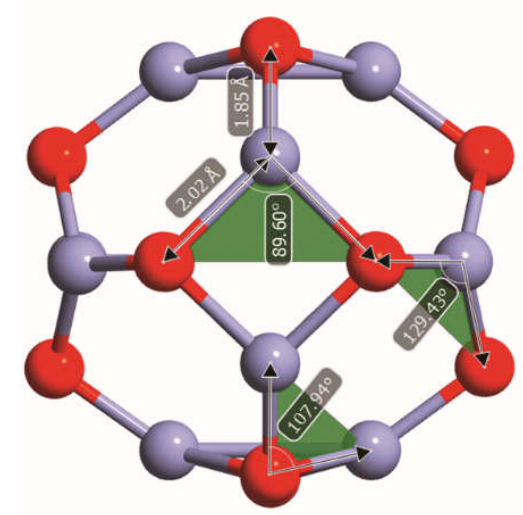

(a)

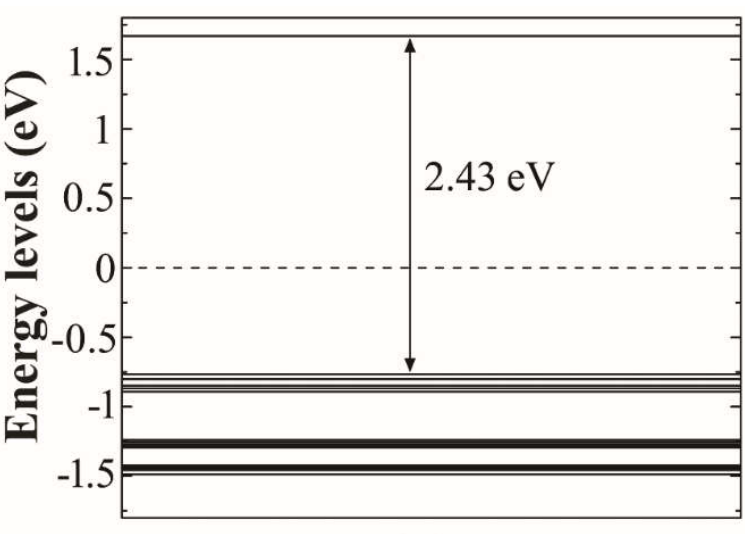

(b)

Figure 6 (a) Optimized $\mathrm{Zn}_{12} \mathrm{O}_{12}$ nanocluster and (b) orbital energy levels diagram of $\mathrm{Zn}_{12} \mathrm{O}_{12}$.

The shortest separation distance between an atom of the adsorbed $\mathrm{Zn}_{12} \mathrm{O}_{12}$ nanocluster and the closest $\mathrm{C}$ atom of the graphene monolayer in the graphene $/ \mathrm{Zn}_{12} \mathrm{O}_{12}$ complex is $2.86 \AA$ which is in agreement with the previous result [53]. The bond length between $\mathrm{Zn}$ and $\mathrm{O}$ is found to be in the range of $1.86 \AA-1.97 \AA$ which concurs with the reported data [207]. From the relaxed geometries of the nano-complexes, it is found that there is no significant structural change of $\mathrm{Zn}_{12} \mathrm{O}_{12}$ nanocluster with respect to their free counterparts whereas graphene undergoes a small local structural distortion.

The interaction between the nanocluster and the graphene layer is expected to be small. However, it was discovered that there is an effective charge transfer between the adsorbed metal/metal oxide nanoparticles and graphene [205]. Our Mulliken population analysis suggests that a net negative charge of $0.104 e$ is transferred from the graphene layer to the nanocluster due to the differences between the electron affinities of the nanocluster and that of graphene. 


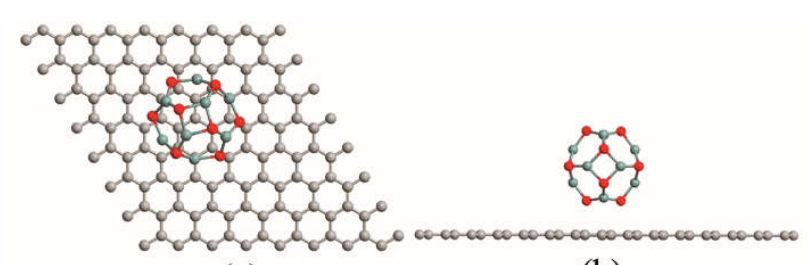

(a)

(b)

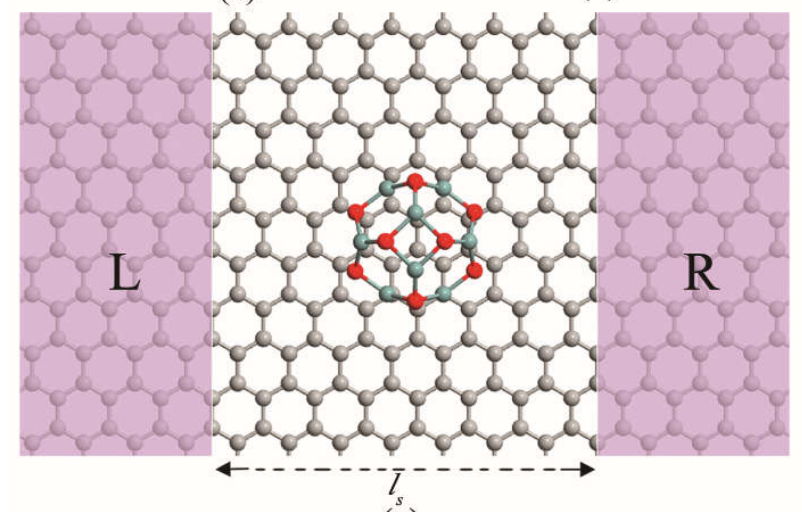

(c)

Figure 6.7 Graphene/ $\mathrm{Zn}_{12} \mathrm{O}_{12}$ atomic structure, (a) top view, (b) side view, where gray as carbon, cyan as zinc and red ball as oxygen, (c) two electrode system of Graphene/ $\mathrm{Zn}_{12} \mathrm{O}_{12}$ heterostructure.

Bandstructure, as well as the density of states (DOS) of the $\mathrm{Zn}_{12} \mathrm{O}_{12}$ decorated graphene complex, is considered to visualize the impact on the electronic property. In neutral graphene which is a zero band gap semiconductor (known as semi-metal), the lowest conduction band and the upper valence band are degenerate at Fermi level $\left(E_{F}\right)$. The graphene band structure and correspondingly DOS are significantly affected by the presence of $\mathrm{Zn}_{12} \mathrm{O}_{12}$ nanoclusters. As can be seen in Figure 6.3, the upper valence band and lower conduction shift apart at $E_{F}$ due to the charge transfer. From the fully relaxed complex structure, it is clear that the graphene-nanocluster interaction region is slightly deformed which could be the reason for breaking the A-B sublattice symmetry at least locally and consequently opening the band gap through a charge transfer bear the Dirac cone. Thus, $14.5 \mathrm{meV}$ band gap is found near to K-point as it could be seen from the Figure 
6.3 insets. This band gap is comparable to the experimental finding in the Ref. [92] (7.36 $\mathrm{meV}$ ) where $\mathrm{ZnO}$ nanoparticles are randomly distributed to tune the band gap by varying the particles size and densities. Moreover, the band gap is close to the previous theoretical finding (25 meV) [53] but did not match exactly. The possible reason might be the vdW interaction which was not included in the previous calculations.

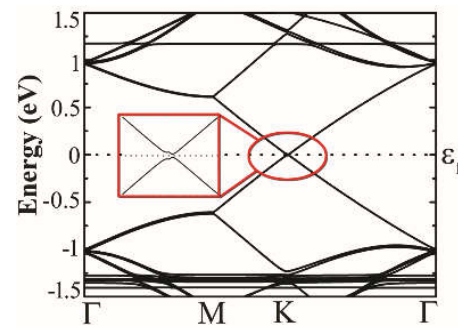

(a)

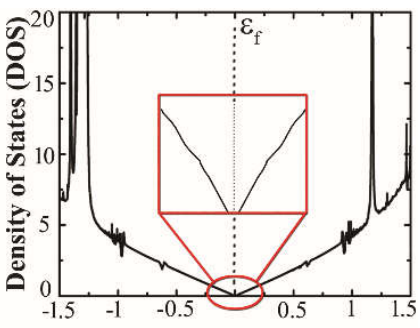

(b)

Figure 6.8 (a) Bandstructure and (b) Density of states of Graphene/ $\mathrm{Zn}_{12} \mathrm{O}_{12}$.

Optical absorption coefficient study from Figure 6.4 (a) shows that the absorbance increased by 1.67 times while maintaining same characteristics as pure graphene covering the visible and infra-red (IR) spectrum. This excellent photo responsiveness of graphene $/ \mathrm{Zn}_{12} \mathrm{O}_{12}$ heterostructure could make it a promising candidate for solar energy and photo detecting devices. Because the $\mathrm{Zn}_{12} \mathrm{O}_{12}$ nanocluster opens a band gap in graphene, it is logical to claim that the structural complex could generate significant current once the device is fabricated and the bias is applied in simple two electrode device. The I-V characteristics of the graphene $/ \mathrm{Zn}_{12} \mathrm{O}_{12}$ show ambipolar behavior where forward and reverse bias produces similar current in the device as it is evident from the Figure 6.4 (b). Instead of being linear like pristine graphene [1], slight nonlinear I-V characteristic is apparent for the energy gap in bias direction because of $\mathrm{Zn}_{12} \mathrm{O}_{12}$ nanocluster induced symmetry breaking in graphene [208]. 


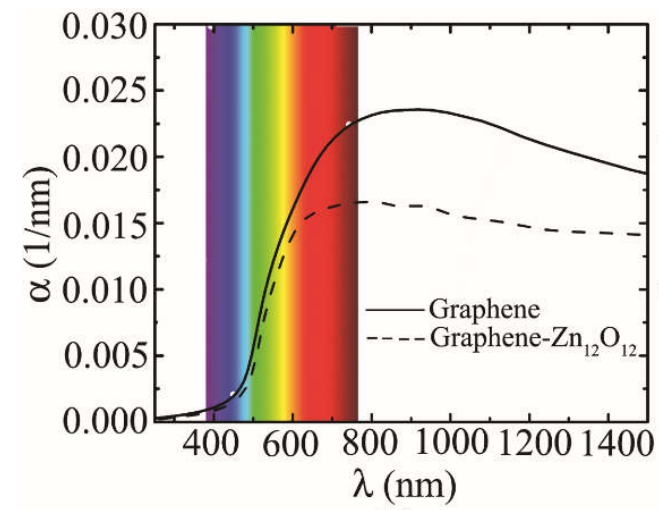

(a)

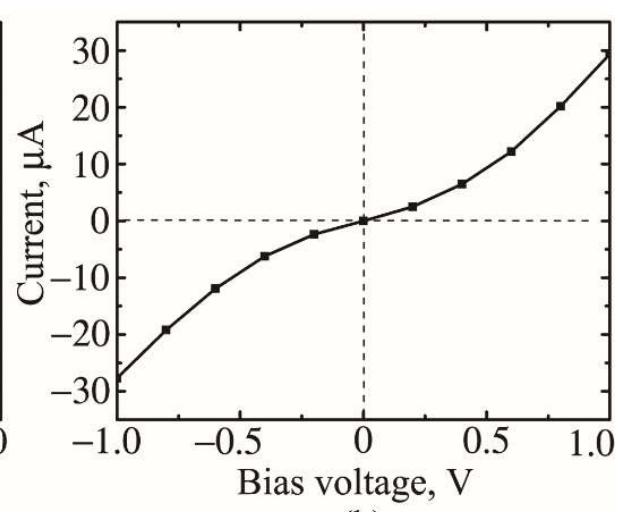

(b)

Figure 6.9 (a) optical absorption coefficient and (b) I-V characteristics of graphene/ $\mathrm{Zn}_{12} \mathrm{O}_{12}$ two electrode system.

\subsection{Conclusion}

First-principles calculation envisaged the possible influence on electronic, transport and optical properties on graphene induced by $\mathrm{Zn}_{12} \mathrm{O}_{12}$ nanocluster on graphene. The results show that nanocluster opens a sizable band gap of the heterostructure as a result of charge transfer. The optical absorption coefficient increases within the visible range and beyond while maintaining the similarity in graphene's characteristics so that the heterostructure could be used for potential solar energy and photo detecting devices. The $I$ - $V$ characteristics of the graphene $/ \mathrm{Zn}_{12} \mathrm{O}_{12}$ heterostructure exhibit slight nonlinear behavior because of the symmetry breaking. Increasing nanocluster density on graphene could provide a practical way to open sizable band gap and tune it for graphene. 


\section{CHAPTER 7}

\section{ZnO nanocluster enhanced optical and electronic properties of graphene/MoS2}

\subsection{Introduction}

It is advantageous to use graphene as a channel material for a photodetector because of its 2D carrier confinement, high residual carrier concentration, high carrier mobility, mechanical flexibility, extremely high thermal conductivity, long phonon mean free path and high optical transparency. The excellent characteristics of graphene have fueled intense research towards its application for highly sensitive and efficient photodetectors and energy harvesting devices, 2D flexible transparent electronics, plasmonics and specialized optoelectronics.

Graphene can absorb light and convert it to photocurrent with an excellent internal quantum efficiency of $100 \%$ because of its long mean-free-path and high Fermi velocity [209]. But graphene absorbs just $2.3 \%$ of light in the visible spectrum. Several mechanisms have been applied to increase light absorption in graphene such as graphene plasmons, microcavities, and metallic plasmons. Photo-voltaic, thermo-electric Seebeck and bolometric effects are identified as possible mechanisms in graphene-based photo-devices. However fast recombination of photo-generated carriers in graphene can reduce device photoresponsivity. The 2D form of $\mathrm{MoS}_{2}$ offers finite direct band gap with extremely high

photoluminescence (PL) because of quantum confinement, whereas its bulk form is an indirect band gap semiconductor. Several groups have reported graphene- $\mathrm{MoS}_{2}$ heterostructure based photodetectors $[56,210,211]$. A photodetector based on a graphene- 
$\mathrm{MoS}_{2}$ heterostructure achieved a photoresponsivity as high as $10^{7} \mathrm{~A} / \mathrm{W}$ and photo gain of $10^{8}$ at room temperature [211]. But those photodetectors do not have wavelength tuning capability, sometimes referred to as gate tunability, with low on/off ratio since graphenes is intrinsically low. To achieve high on/off switching ratio with tuning capability in the layered heterostructure, graphene nanoribbons or gapped graphene can be used [56, 210, 211]. The photodetector must have high responsivity at the operating wavelength, high response speed, and low noise. In addition, the photodetector should be compact, use low biasing voltage or current, and be reliable under the required operating condition [56, 212].

Photodetectors are semiconductor devices that convert optical signals into electrical signals. The operation of a photodetector involves three steps: carrier generation by incident light, carrier transport and/or multiplication by current gain mechanism, and interaction of the current with an external circuit to provide the output signal.

Although graphene has high mobility characteristics, its poor absorption efficiency and fast recombination are detrimental in building highly responsive and efficient photo devices. On the other hand, bulk $\mathrm{MoS}_{2}$ is an indirect band gap $(1.2 \mathrm{eV})$ semiconductor, but when reduced to the monolayer, it becomes a direct band gap semiconductor with a band gap of $1.8 \mathrm{eV}$. This results in a high absorption coefficient and efficient electron-hole pair generation under photoexcitation in monolayer $\mathrm{MoS}_{2}$. Compared to bulk $\mathrm{MoS}_{2}$, a quantum luminescence yield of $10^{4}$ fold was observed for monolayer $\mathrm{MoS}_{2}$ leading to several groups trying to improve photodetector efficiency using this novel material [213-216]. Efforts have been made to improve the photodetector's responsivity by stacking graphene and with $\mathrm{MoS}_{2}$ to create multifunctional high-performance devices [130, 217-222]. Coupling 
graphene with $\mathrm{MoS}_{2}$ is one type of heterostructure taking advantage of the high mobility of graphene and high absorption capability of $\mathrm{MoS}_{2}$ to fabricate a highly responsive photodetector [211]. Graphene is p-doped under ambient conditions. This is caused by the doping effect from the transfer process, adsorbed moisture/oxygen and substrate impurities $[49,223]$. The hole concentration in graphene decreases from $6 \times 10^{12} \mathrm{~cm}^{-2}$ to $2 \times 10^{12} \mathrm{~cm}^{-2}$ and the resistance significantly increases when graphene is in contact with $\mathrm{MoS}_{2}$, suggesting that electrons move from $\mathrm{MoS}_{2}$ to graphene.

A large exciton binding energy of $\sim 60 \mathrm{meV}$ (2.4 times of the room-temperature thermal energy) and a wide band gap (3.37 eV) of $\mathrm{ZnO}$ [91] make it useful to open and tune band gap in graphene $[75,92]$. Moreover, the amalgamation of $\mathrm{ZnO}$ with graphene could open a new route to heterostructure for optoelectronic devices such as solar cells, field emission, displays, sensors, light emitting, photocatalysis and detection devices in UV-visible spectral range [201, 202]. It has already been proven for rapid and inexpensive synthesis of $\mathrm{ZnO}$ nanoparticles using available cost-effective standard semiconductor device fabrication technologies [91]. In addition, DFT calculation investigated that $\mathrm{Zn}_{12} \mathrm{O}_{12}$ nanocluster imitates nanoseeds on graphene [203].

\subsection{Methodology}

First-principle Calculations are performed using DFT implemented in Atomistix ToolKit (ATK) package [148]. The Generalized Gradient Approximation of Perdew-BurkeErnzerhof (GGA-PBE) with a double- $\zeta$ polarized basis set $[121,130,149]$ is used for exchange-correlation functional. Heterostructure made of $\mathrm{Zn}_{12} \mathrm{O}_{12} /$ graphene/ $\mathrm{MoS}_{2}$, 
consisting of a $\mathrm{Zn}_{12} \mathrm{O}_{12}$ nanocluster, $5 \times 5$ supercell of graphene and $4 \times 4$ (as shown in Figure 6.1(a) and (b)), has been fully relaxed (lattice constant and atomic positions) until the maximum atomic force and stress on each atom are less than $0.01 \mathrm{eV} / \AA, 0.05 \mathrm{eV} / \AA^{3}$, respectively. A grid mesh cut-off energy of $75 \mathrm{Ha}$ and $1 \times 3 \times 3$ k-points for the integration of the first Brillouin zone are chosen. A vacuum separation of $25 \AA$ perpendicular to the structure is considered to form a free-standing ultrathin film to suppress adjacent images interactions. The Grimme vdW correction (DFT-D2) is included in the calculations to account long-range van der Waals (vdW) interactions [127]. Finally, the k-points have been increased to $1 \times 101 \times 101$ for electronic, transport, optical properties calculations.

A fast Fourier transform (FFT) solver is used to solve Poisson equation and nonequilibrium green's function (NEGF) is combined GGA/PBE for optimization and transport properties calculations.

The schematic of two electrode system made of $\mathrm{Zn}_{12} \mathrm{O}_{12} /$ graphene/ $\mathrm{MoS}_{2}$ heterostructure presented in Figure 7.1(c). The electrical current through the device under non-equilibrium conditions, i.e. for a finite bias voltage $\left(V_{\text {Bias }}\right)$, can be calculated using the Landauer formula as follows [204]

$$
I\left(V_{\text {Bias }}\right)=\frac{2 e}{h} \int_{-\infty}^{+\infty} T\left(\varepsilon, V_{\text {Bias }}\right)\left[f\left(\varepsilon-\mu_{\mathrm{L}}\right)-f\left(\varepsilon-\mu_{\mathrm{R}}\right)\right] \mathrm{d} \varepsilon
$$

Where $f$ is the Fermi-Dirac distribution function, $\mu_{\mathrm{L}, \mathrm{R}}=E_{\mathrm{F}} \pm e V_{\text {Bias }} / 2$ represent the chemical potentials of the left and right electrodes, and $T\left(\varepsilon, V_{\text {Bias }}\right)$ is the energy and voltage 
dependent transmission function. The transport setup consists of two graphene/ $\mathrm{MoS}_{2}$ electrodes of $2 \AA$ and $\mathrm{Zn}_{12} \mathrm{O}_{12} /$ graphene/MoS 2 structure as central region. The supercell was oriented such way that transport direction connecting $\mathrm{L}$ and $\mathrm{R}$ was aligned parallel to the armchair direction within the structure, as shown in Figure 7.1(c).The optical absorption spectrum is calculated using the Kubo-Greenwood formula [205]. The susceptibility tensor in this method given as follows

$$
\chi_{i j}(\omega)=-\frac{e^{2} \hbar^{4}}{m^{2} \varepsilon_{0} V \omega^{2}} \sum_{n n} \frac{f\left(E_{m}\right)-f\left(E_{n}\right)}{E_{n n}-\hbar \omega-i \hbar \Gamma} \times \pi_{n n}^{i} \pi_{m n}^{j}
$$

Where, $\pi_{n m}^{i}$ means $i$-component of the dipole matrix element between $n$ and $m$ states, $\Gamma$ is the broadening, $V$ is the volume, $f$ is the Fermi function and $E_{m}\left(E_{n}\right)$ corresponds to eigenvalues of $m(n)$ state. The dielectric constant $\left(\varepsilon_{r}\right)$ can be written in terms of the susceptibility tensor $\chi(\omega)$, using linear response theory as follows

$$
\varepsilon_{r}(\omega)=1+\chi(\omega)
$$

The refractive index, $n$, is related to the complex dielectric constant through

$$
n+i \kappa=\sqrt{\varepsilon_{r}}
$$

Here, $\kappa$ is the extinction coefficient. Finally, the optical absorption coefficient is related to the extinction coefficient through [206]

$$
\alpha_{a}=2 \frac{\omega}{C} \kappa
$$




\subsection{Results and Discussion}

The shortest separation distance between an atom of the adsorbed $\mathrm{Zn}_{12} \mathrm{O}_{12}$ nanocluster and the closest $\mathrm{C}$ atom of the graphene monolayer in the $\mathrm{Zn}_{12} \mathrm{O}_{12}$ /graphene/ $\mathrm{MoS}_{2}$ complex is $2.57 \AA$. The bond length between $\mathrm{Zn}$ and $\mathrm{O}$ is found to be in the range of $1.86 \AA-1.99 \AA$ which concurs with the reported data [207]. In the $\mathrm{Zn}_{12} \mathrm{O}_{12}$ nanocluster, the optimized length of $\mathrm{Zn}-\mathrm{O}$ bonds are $1.88 \AA$ which is shared between two hexagon rings and $1.98 \AA$ which is shared between a tetragon and hexagon ring.

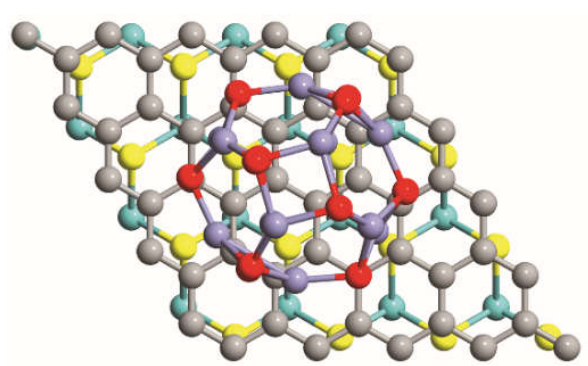

(a)

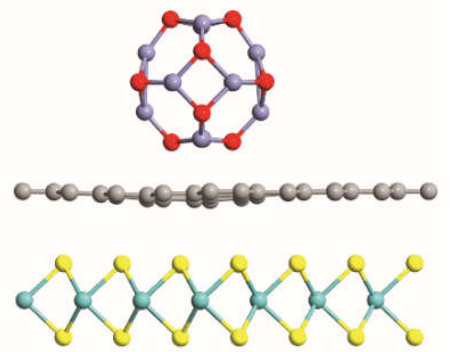

(b)

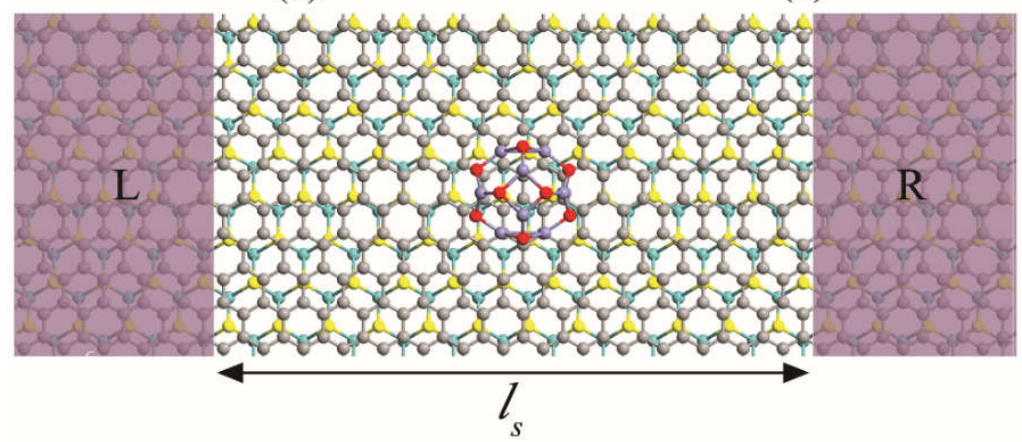

(c)

Figure 7.1 $\mathrm{Zn}_{12} \mathrm{O}_{12} /$ Graphene/ $\mathrm{MoS}_{2}$ atomic structure, (a) top view, (b) side view, where gray as carbon, cyan as zinc and red ball as oxygen, (c) two electrode system of $\mathrm{Zn}_{12} \mathrm{O}_{12} /$ Graphene/ $\mathrm{MoS}_{2}$ heterostructure.

From the relaxed geometries of the nano-complexes, it is found that there is no significant structural change of $\mathrm{Zn}_{12} \mathrm{O}_{12}$ nanocluster with respect to their free counterparts whereas 
graphene undergoes a small local structural distortion and $\mathrm{MoS}_{2}$ has neglizible structural distortion.

Bandstructure, as well as the density of states (DOS) of the $\mathrm{Zn}_{12} \mathrm{O}_{12}$ decorated graphene complex, is considered to visualize the impact on the electronic property. In neutral graphene which is a zero band gap semiconductor (known as semi-metal), the lowest conduction band and the upper valence band are degenerate at Fermi level $\left(E_{F}\right)$. The graphene band structure and correspondingly DOS are significantly affected by the presence of $\mathrm{Zn}_{12} \mathrm{O}_{12}$ nanoclusters. As can be seen in Figure 7.2, the upper valence band and lower conduction shift apart at $E_{F}$ due to the charge transfer.

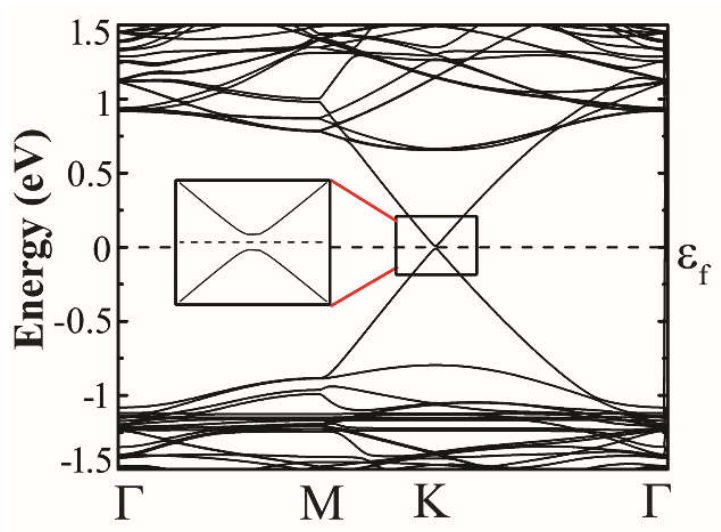

(a)

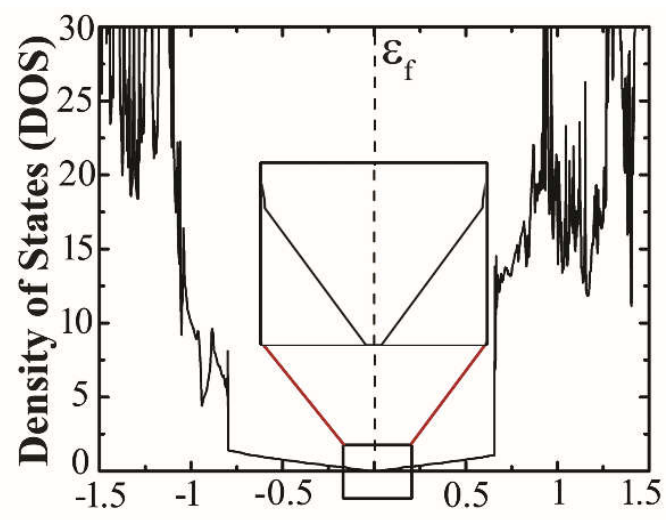

(b)

Figure 7.2 (a) Bandstructure and (b) Density of states of $\mathrm{Zn}_{12} \mathrm{O}_{12} / \mathrm{Graphene} / \mathrm{MoS}_{2}$

From the fully relaxed complex structure, it is clear that the graphene-nanocluster interaction region is slightly deformed which could be the reason for breaking the A-B sublattice symmetry at least locally and consequently opening the band gap through a charge transfer near the Dirac cone. Our Mulliken population analysis calculates a net 
negative charge of $0.104 e$ is being transferred to the nanocluster from the underlyng graphene/MoS . Thus, $20.48 \mathrm{meV}$ band gap is found near to $\mathrm{K}$-point as it could be seen from the Figure 7.2 insets which are larger compared to the Ref [224] while graphene/ $\mathrm{MoS}_{2}$ heterostructure calculation shows bandgap of $40.65 \mathrm{meV}$. But, $\mathrm{Zn}_{12} \mathrm{O}_{12} / \mathrm{Graphene} / \mathrm{MoS}_{2}$ heterostructure shows a wide flat band gap around this particular point. The flat band potential could enhance the photoconversion efficiency through recombination process [225].

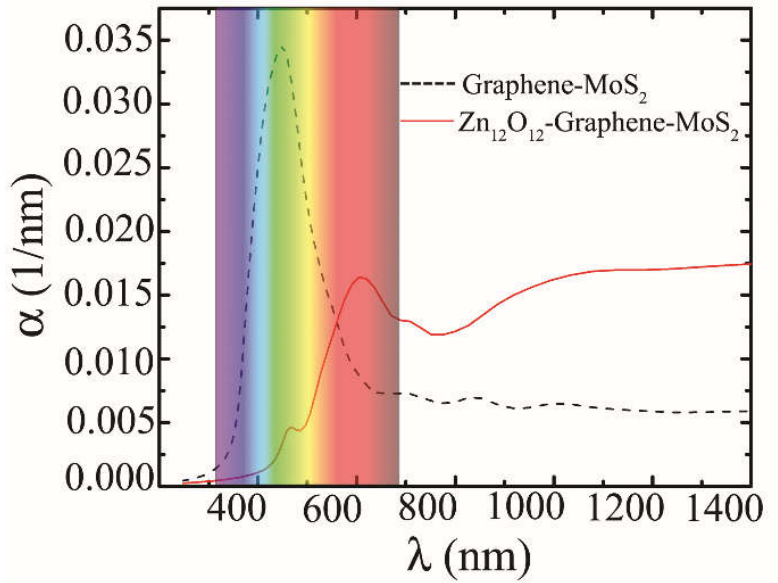

(a)

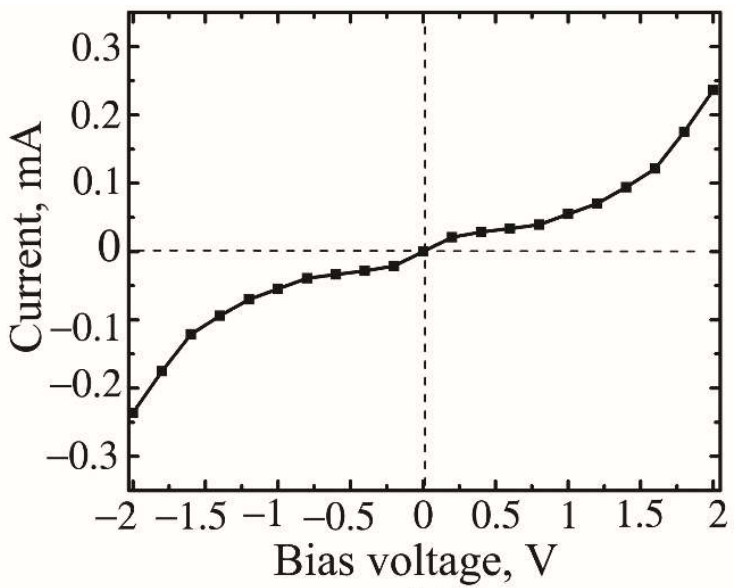

(b)

Figure 7.3 (a) optical absorption coefficient and (b) I-V characteristics of $\mathrm{Zn}_{12} \mathrm{O}_{12} /$ Graphene/ $\mathrm{MoS}_{2}$ two electrode system.

Optical absorption coefficient study from Figure 7.3 (a) shows that the $\mathrm{Zn}_{12} \mathrm{O}_{12} /$ Graphene/ $\mathrm{MoS}_{2}$ nanocomplex absorbance signifacntly reduced in the $400-600 \mathrm{~nm}$ spectrum window and increased by 2 times afterwards compared to Graphene/ $\mathrm{MoS}_{2}$ heterostructure. This excellent photo responsiveness of $\mathrm{Zn}_{12} \mathrm{O}_{12} /$ Graphene/ $\mathrm{MoS}_{2}$ heterostructure could make it a promising candidate for infra-red photo detecting devices. Because the $\mathrm{Zn}_{12} \mathrm{O}_{12}$ nanocluster opens a band gap in graphene, it is logical to claim that the structural complex could generate significant current once the device is fabricated and 
the bias is applied in simple two electrode device. The I-V characteristics of the $\mathrm{Zn}_{12} \mathrm{O}_{12} /$ Graphene/ $\mathrm{MoS}_{2}$ show ambipolar behavior where forward and reverse bias produces similar current in the device as it is evident from the Figure 7.3 (b). Instead of being linear like pristine graphene [1], nonlinear I-V characteristic is apparent for the energy gap in bias direction because of $\mathrm{Zn}_{12} \mathrm{O}_{12}$ nanocluster induced symmetry breaking in graphene [208].

\subsection{Conclusion}

It has been shown that $\mathrm{Zn}_{12} \mathrm{O}_{12}$ nanocluster could enhance the electronic properties of graphene/MoS 2 complexes while providing larger flat band gap. Electronic properties found in this research suggested the possibility of using such heterostructure in promising energy harvesting, photodetection devices and photolysis of molecules for the future cheapest energy delivery system. 


\section{CHAPTER 8}

\section{Conclusions and Future Direction}

\subsection{Conclusions}

Electronic and magnetic properties of edge-functionalized (-H, -OH, -F, -Cl) AGeNRs and ZGeNRs were investigated using first principle calculations based on DFT. It was found that the halogenated structures are more stable than hydrogenated or hydroxide structures though stability decreases with increasing atomic number of halogens. Moreover, halogens still ensure more stable structures than other functionalized atoms in any case. Quantum confinement was also retained even when functionalized with other atoms rather than hydrogenated only.

This study showed that the energy band gap changes are strongly dependent on the armchair nanoribbon's width, edge atom functionalization and the number of functionalizing atoms at the edges. Edge functionalization can be used for energy band gap tuning (0.01-0.84 eV) of AGeNRs. Depending on the type of edge functionalization, the same band gap with wider ribbons can be achieved.

Formation energy studies revealed, regardless of impurity atoms ( $\mathrm{N}$ or B) in AGeNRs or ZGeNRs, dopants preferred to be on the edges. Single atom doped AGeNRs at one edge becomes metallic due to the appearance of a half-filled impurity atom induced band which enables them to be used in negative differential resistance devices. Single atom doping (N or B) suppresses the spin polarization of the doped edges transforming it from an AFM ground state to FM. Lastly, formation and edge free energy studies revealed the feasibility of chemical synthetization of edge functionalized germanene. 
First-principles calculation envisaged the possible influence on electronic, transport and optical properties on graphene induced by $\mathrm{Zn}_{12} \mathrm{O}_{12}$ nanocluster on graphene. The results show that nanocluster opens a sizable band gap of the heterostructure as a result of charge transfer. The optical absorption coefficient increases within the visible range and beyond while maintaining the similarity in graphene's characteristics so that the heterostructure could be used for potential solar energy and photo detecting devices. The $I-V$ characteristics of the graphene/ $\mathrm{Zn}_{12} \mathrm{O}_{12}$ heterostructure exhibit slight nonlinear behavior because of the symmetry breaking. Increasing nanocluster density on graphene could provide a practical way to open sizable band gap and tune it for graphene.

\subsection{Future Direction}

1. Building $\mathrm{Zn}_{12} \mathrm{O}_{12}$ /graphene energy harvesting and photo-detecting device. By varying density of $\mathrm{Zn}_{12} \mathrm{O}_{12}$, we could tune the bandgap of graphene to integrate into other heterostructure devices with enhanced electronic and optical performances.

2. Fabricating photodetector using monolayer $\mathrm{MoS}_{2}$ with band gap engineered graphene which could cover a wider spectrum than available photodetector with the excellent current on/off ratio.

3. Building gas sensors out of edge functionalized, nanoparticle engineered 2D materials which could offer excellent sensitivity in presence of toxic gases.

4. Predicted edge functionalized, doped germanene and other possible 2D materials should be explored to supplement the "2D building blocks" for future "designer structures" with unique and controlled properties for the prompt advancement of many 
technologies, such as electronics, photonics, photo-catalysis and even, molecular sensing, separation, and storage etc. 


\section{References}

[1] K. S. Novoselov, A. K. Geim, S. V. Morozov, D. Jiang, Y. Zhang, S. V. Dubonos, I. V. Grigorieva, and A. A. Firsov, "Electric field effect in atomically thin carbon films," science, vol. 306, no. 5696, pp. 666-669, 2004.

[2] K. I. Bolotin, K. Sikes, Z. Jiang, M. Klima, G. Fudenberg, J. Hone, P. Kim, and H. Stormer, "Ultrahigh electron mobility in suspended graphene," Solid State Communications, vol. 146, no. 9, pp. 351-355, 2008.

[3] A. A. Balandin, S. Ghosh, W. Bao, I. Calizo, D. Teweldebrhan, F. Miao, and C. N. Lau, "Superior thermal conductivity of single-layer graphene," Nano letters, vol. 8, no. 3, pp. 902-907, 2008.

[4] A. Kuzmenko, E. Van Heumen, F. Carbone, and D. Van Der Marel, "Universal optical conductance of graphite," Physical review letters, vol. 100, no. 11, p. 117401, 2008.

[5] C. Lee, X. Wei, J. W. Kysar, and J. Hone, "Measurement of the elastic properties and intrinsic strength of monolayer graphene," science, vol. 321, no. 5887, pp. 385-388, 2008.

[6] K. S. Novoselov, A. K. Geim, S. Morozov, D. Jiang, M. Katsnelson, I. Grigorieva, S. Dubonos, and A. Firsov, "Two-dimensional gas of massless Dirac fermions in graphene," nature, vol. 438, no. 7065, pp. 197-200, 2005.

[7] B. Radisavljevic, A. Radenovic, J. Brivio, i. V. Giacometti, and A. Kis, "Single-layer MoS2 transistors," Nature nanotechnology, vol. 6, no. 3, pp. 147-150, 2011.

[8] T. Aizawa, S. Suehara, and S. Otani, "Silicene on zirconium carbide (111)," The Journal of Physical Chemistry C, vol. 118, no. 40, pp. 23049-23057, 2014.

[9] B. Aufray, A. Kara, S. Vizzini, H. Oughaddou, C. Léandri, B. Ealet, and G. Le Lay, "Graphene-like silicon nanoribbons on $\operatorname{Ag}(110)$ : A possible formation of silicene," Applied Physics Letters, vol. 96, no. 18, p. 183102, 2010.

[10]B. Feng, Z. Ding, S. Meng, Y. Yao, X. He, P. Cheng, L. Chen, and K. Wu, "Evidence of silicene in honeycomb structures of silicon on $\mathrm{Ag}$ (111)," Nano Letters, vol. 12, no. 7, pp. 3507-3511, 2012.

[11]M. Dávila, L. Xian, S. Cahangirov, A. Rubio, and G. Le Lay, "Germanene: a novel two-dimensional germanium allotrope akin to graphene and silicene," New Journal of Physics, vol. 16, no. 9, p. 095002, 2014. 
[12]L. Li, Y. Yu, G. J. Ye, Q. Ge, X. Ou, H. Wu, D. Feng, X. H. Chen, and Y. Zhang, "Black phosphorus field-effect transistors," Nature nanotechnology, vol. 9, no. 5, pp. 372-377, 2014.

[13]S. Cahangirov, M. Topsakal, E. Aktürk, H. Şahin, and S. Ciraci, "Two-and onedimensional honeycomb structures of silicon and germanium," Physical review letters, vol. 102, no. 23, p. 236804, 2009.

[14]H. Şahin, S. Cahangirov, M. Topsakal, E. Bekaroglu, E. Akturk, R. T. Senger, and S. Ciraci, "Monolayer honeycomb structures of group-IV elements and III-V binary compounds: First-principles calculations," Physical Review B, vol. 80, no. 15, p. $155453,2009$.

[15]L. Meng, Y. Wang, L. Zhang, S. Du, R. Wu, L. Li, Y. Zhang, G. Li, H. Zhou, and W. A. Hofer, "Buckled silicene formation on Ir (111)," Nano letters, vol. 13, no. 2, pp. 685-690, 2013.

[16]A. Fleurence, R. Friedlein, T. Ozaki, H. Kawai, Y. Wang, and Y. Yamada-Takamura, "Experimental evidence for epitaxial silicene on diboride thin films," Physical review letters, vol. 108, no. 24, p. 245501, 2012.

[17]K. V. Emtsev, A. Bostwick, K. Horn, J. Jobst, G. L. Kellogg, L. Ley, J. L. McChesney, T. Ohta, S. A. Reshanov, and J. Röhrl, "Towards wafer-size graphene layers by atmospheric pressure graphitization of silicon carbide," Nature materials, vol. 8, no. 3, pp. 203-207, 2009.

[18]C. Berger, Z. Song, X. Li, X. Wu, N. Brown, C. Naud, D. Mayou, T. Li, J. Hass, and A. N. Marchenkov, "Electronic confinement and coherence in patterned epitaxial graphene," Science, vol. 312, no. 5777, pp. 1191-1196, 2006.

[19]Y. Hernandez, V. Nicolosi, M. Lotya, F. M. Blighe, Z. Sun, S. De, I. McGovern, B. Holland, M. Byrne, and Y. K. Gun'Ko, "High-yield production of graphene by liquidphase exfoliation of graphite," Nature nanotechnology, vol. 3, no. 9, pp. 563-568, 2008.

[20]C. Rao, U. Maitra, and H. Matte, "Synthesis, characterization, and selected properties of graphene," Graphene; Wiley-VCH Verlag GmbH \& Co. KGaA: Weinheim, Germany, pp. 1-47, 2012.

[21]D. Wei, Y. Liu, Y. Wang, H. Zhang, L. Huang, and G. Yu, "Synthesis of N-doped graphene by chemical vapor deposition and its electrical properties," Nano letters, vol. 9, no. 5, pp. 1752-1758, 2009. 
[22]X. Li, W. Cai, J. An, S. Kim, J. Nah, D. Yang, R. Piner, A. Velamakanni, I. Jung, and E. Tutuc, "Large-area synthesis of high-quality and uniform graphene films on copper foils," Science, vol. 324, no. 5932, pp. 1312-1314, 2009.

[23]A. Reina, X. Jia, J. Ho, D. Nezich, H. Son, V. Bulovic, M. S. Dresselhaus, and J. Kong, "Large area, few-layer graphene films on arbitrary substrates by chemical vapor deposition," Nano letters, vol. 9, no. 1, pp. 30-35, 2008.

[24]K. S. Kim, Y. Zhao, H. Jang, S. Y. Lee, J. M. Kim, K. S. Kim, J. H. Ahn, P. Kim, J. Y. Choi, and B. H. Hong, "Large-scale pattern growth of graphene films for stretchable transparent electrodes," nature, vol. 457, no. 7230, pp. 706-710, 2009.

[25]Q. Yu, J. Lian, S. Siriponglert, H. Li, Y. P. Chen, and S.-S. Pei, "Graphene segregated on Ni surfaces and transferred to insulators," Applied Physics Letters, vol. 93, no. 11, p. 113103, 2008.

[26]N. J. Roome and J. D. Carey, "Beyond graphene: Stable elemental monolayers of silicene and germanene," ACS applied materials \& interfaces, vol. 6, no. 10, pp. 7743$7750,2014$.

[27]L. Li, S. z. Lu, J. Pan, Z. Qin, Y. q. Wang, Y. Wang, G. y. Cao, S. Du, and H. J. Gao, "Buckled germanene formation on Pt (111)," Advanced Materials, vol. 26, no. 28, pp. 4820-4824, 2014.

[28]H. Oughaddou, S. Sawaya, J. Goniakowski, B. Aufray, G. Le Lay, J. Gay, G. Tréglia, J. Bibérian, N. Barrett, and C. Guillot, "Ge/Ag (111) semiconductor-on-metal growth: Formation of an Ag 2 Ge surface alloy," Physical Review B, vol. 62, no. 24, p. 16653, 2000 .

[29]M. Derivaz, D. Dentel, R. Stephan, M.-C. Hanf, A. Mehdaoui, P. Sonnet, and C. Pirri, "Continuous germanene layer on Al (111)," Nano letters, vol. 15, no. 4, pp. 25102516, 2015.

[30]E. Bianco, S. Butler, S. Jiang, O. D. Restrepo, W. Windl, and J. E. Goldberger, "Stability and exfoliation of germanane: a germanium graphane analogue," Acs Nano, vol. 7, no. 5, pp. 4414-4421, 2013.

[31]P. Vogt, P. De Padova, C. Quaresima, J. Avila, E. Frantzeskakis, M. C. Asensio, A. Resta, B. Ealet, and G. Le Lay, "Silicene: compelling experimental evidence for graphenelike two-dimensional silicon," Physical review letters, vol. 108, no. 15, p. $155501,2012$.

[32]D. Chiappe, E. Scalise, E. Cinquanta, C. Grazianetti, B. van den Broek, M. Fanciulli, M. Houssa, and A. Molle, "Two-Dimensional Si Nanosheets with Local Hexagonal 
Structure on a $\mathrm{MoS}_{2}$ Surface," Advanced Materials, vol. 26, no. 13, pp. 2096-2101, 2014.

[33]E. Varrla, C. Backes, K. R. Paton, A. Harvey, Z. Gholamvand, J. McCauley, and J. N. Coleman, "Large-scale production of size-controlled MoS2 nanosheets by shear exfoliation," Chemistry of Materials, vol. 27, no. 3, pp. 1129-1139, 2015.

[34]J. N. Coleman, M. Lotya, A. O’Neill, S. D. Bergin, P. J. King, U. Khan, K. Young, A. Gaucher, S. De, and R. J. Smith, "Two-dimensional nanosheets produced by liquid exfoliation of layered materials," Science, vol. 331, no. 6017, pp. 568-571, 2011.

[35]Y. Yao, Z. Lin, Z. Li, X. Song, K.-S. Moon, and C.-p. Wong, "Large-scale production of two-dimensional nanosheets," Journal of Materials Chemistry, vol. 22, no. 27, pp. 13494-13499, 2012.

[36] K. S. Novoselov, V. Fal, L. Colombo, P. Gellert, M. Schwab, and K. Kim, "A roadmap for graphene," Nature, vol. 490, no. 7419, pp. 192-200, 2012.

[37]M. Y. Han, B. Özyilmaz, Y. Zhang, and P. Kim, "Energy band-gap engineering of graphene nanoribbons," Physical review letters, vol. 98, no. 20, p. 206805, 2007.

[38]T. Ohta, A. Bostwick, T. Seyller, K. Horn, and E. Rotenberg, "Controlling the electronic structure of bilayer graphene," Science, vol. 313, no. 5789, pp. 951-954, 2006.

[39]J. Oostinga, H. Heersche, X. Liu, A. Morpurgo, and L. Vandersypen, "Gate-tunable band-gap in bilayer graphene devices," Nature Materials, vol. 7, p. 151, 2008.

[40]D. C. Elias, R. R. Nair, T. Mohiuddin, S. Morozov, P. Blake, M. Halsall, A. Ferrari, D. Boukhvalov, M. Katsnelson, and A. Geim, "Control of graphene's properties by reversible hydrogenation: evidence for graphane," Science, vol. 323, no. 5914, pp. 610-613, 2009.

[41]F. Zhao, T. Thuong Nguyen, M. Golsharifi, S. Amakubo, K. Loh, and R. B. Jackman, "Electronic properties of graphene-single crystal diamond heterostructures," Journal of Applied Physics, vol. 114, no. 5, p. 053709, 2013.

[42]S. Morozov, K. Novoselov, M. Katsnelson, F. Schedin, L. Ponomarenko, D. Jiang, and A. Geim, "Strong suppression of weak localization in graphene," Physical review letters, vol. 97, no. 1, p. 016801, 2006.

[43]Y. Zhang, V. W. Brar, C. Girit, A. Zettl, and M. F. Crommie, "Origin of spatial charge inhomogeneity in graphene," Nature Physics, vol. 5, no. 10, pp. 722-726, 2009. 
[44]Y. Niimi, T. Matsui, H. Kambara, K. Tagami, M. Tsukada, and H. Fukuyama, "Scanning tunneling microscopy and spectroscopy of the electronic local density of states of graphite surfaces near monoatomic step edges," Physical Review B, vol. 73, no. 8, p. $085421,2006$.

[45]T. Wehling, A. Balatsky, M. Katsnelson, A. Lichtenstein, K. Scharnberg, and R. Wiesendanger, "Local electronic signatures of impurity states in graphene," Physical Review B, vol. 75, no. 12, p. 125425, 2007.

[46]S. M. Aghaei, M. M. Monshi, I. Torres, and I. Calizo, "Edge functionalization and doping effects on the stability, electronic and magnetic properties of silicene nanoribbons," RSC Advances, vol. 6, no. 21, pp. 17046-17058, 2016.

[47]M. Monshi, S. Aghaei, and I. Calizo, "Edge functionalized germanene nanoribbons: impact on electronic and magnetic properties," RSC Advances, vol. 7, no. 31, pp. 18900-18908, 2017.

[48]T. T. Jia, X. Y. Fan, M. M. Zheng, and G. Chen, "Silicene nanomeshes: bandgap opening by bond symmetry breaking and uniaxial strain," Scientific reports, vol. 6, p. 20971, 2016.

[49] W. Zhang, C. T. Lin, K. K. Liu, T. Tite, C. Y. Su, C. H. Chang, Y. H. Lee, C. W. Chu, K. H. Wei, and J. L. Kuo, "Opening an electrical band gap of bilayer graphene with molecular doping," ACS nano, vol. 5, no. 9, pp. 7517-7524, 2011.

[50]P. Wagner, C. P. Ewels, J. J. Adjizian, L. Magaud, P. Pochet, S. Roche, A. LopezBezanilla, V. V. Ivanovskaya, A. Yaya, and M. Rayson, "Band gap engineering via edge-functionalization of graphene nanoribbons," The Journal of Physical Chemistry C, vol. 117, no. 50, pp. 26790-26796, 2013.

[51]J. Bai, X. Zhong, S. Jiang, Y. Huang, and X. Duan, "Graphene nanomesh," Nature nanotechnology, vol. 5, no. 3, pp. 190-194, 2010.

[52]F. Pan, Y. Wang, K. Jiang, Z. Ni, J. Ma, J. Zheng, R. Quhe, J. Shi, J. Yang, and C. Chen, "Silicene nanomesh," Scientific reports, vol. 5, p. 9075, 2015.

[53]B. Das, B. Choudhury, A. Gomathi, A. K. Manna, S. Pati, and C. Rao, "Interaction of inorganic nanoparticles with graphene," ChemPhysChem, vol. 12, no. 5, pp. 937-943, 2011.

[54]L. Kulyuk, L. Charron, and E. Fortin, "Radiative properties of $\mathrm{MoS}_{2}$ layered crystals," Physical Review B, vol. 68, no. 7, p. 075314, 2003. 
[55]Q. H. Wang, K. Kalantar-Zadeh, A. Kis, J. N. Coleman, and M. S. Strano, "Electronics and optoelectronics of two-dimensional transition metal dichalcogenides," Nature nanotechnology, vol. 7, no. 11, pp. 699-712, 2012.

[56]P. Vabbina, N. Choudhary, A.-A. Chowdhury, R. Sinha, M. Karabiyik, S. Das, W. Choi, and N. Pala, "Highly sensitive wide bandwidth photodetector based on internal photoemission in CVD grown p-type MoS2/graphene Schottky junction," ACS applied materials \& interfaces, vol. 7, no. 28, pp. 15206-15213, 2015.

[57]H. Liu, A. T. Neal, Z. Zhu, Z. Luo, X. Xu, D. Tománek, and D. Y. Peide, "Phosphorene: an unexplored 2D semiconductor with a high hole mobility," 2014.

[58]K. Novoselov and A. C. Neto, "Two-dimensional crystals-based heterostructures: materials with tailored properties," Physica Scripta, vol. 2012, no. T146, p. 014006, 2012.

[59]C. Dean, A. Young, L. Wang, I. Meric, G.-H. Lee, K. Watanabe, T. Taniguchi, K. Shepard, P. Kim, and J. Hone, "Graphene based heterostructures," Solid State Communications, vol. 152, no. 15, pp. 1275-1282, 2012.

[60]L. Britnell, R. Gorbachev, R. Jalil, B. Belle, F. Schedin, A. Mishchenko, T. Georgiou, M. Katsnelson, L. Eaves, and S. Morozov, "Field-effect tunneling transistor based on vertical graphene heterostructures," Science, vol. 335, no. 6071, pp. 947-950, 2012.

[61]W. J. Yu, Z. Li, H. Zhou, Y. Chen, Y. Wang, Y. Huang, and X. Duan, "Vertically stacked multi-heterostructures of layered materials for logic transistors and complementary inverters," Nature materials, vol. 12, no. 3, pp. 246-252, 2013.

[62]G. K. Teal, "Single crystals of germanium and silicon-Basic to the transistor and integrated circuit," IEEE Transactions on electron devices, vol. 23, no. 7, pp. 621-639, 1976.

[63]M. A. Green and M. J. Keevers, "Optical properties of intrinsic silicon at $300 \mathrm{~K}$," Progress in Photovoltaics: Research and Applications, vol. 3, no. 3, pp. 189-192, 1995.

[64]P. R. Wallace, "The band theory of graphite," Physical Review, vol. 71, no. 9, p. 622, 1947.

[65] A. K. Geim and K. S. Novoselov, "The rise of graphene," Nature materials, vol. 6, no. 3, pp. 183-191, 2007.

[66]J. Kedzierski, P. L. Hsu, P. Healey, P. W. Wyatt, C. L. Keast, M. Sprinkle, C. Berger, and W. A. De Heer, "Epitaxial graphene transistors on SiC substrates," IEEE Transactions on Electron Devices, vol. 55, no. 8, pp. 2078-2085, 2008. 
[67]Y. Wu, P. Ye, M. A. Capano, Y. Xuan, Y. Sui, M. Qi, J. A. Cooper, T. Shen, D. Pandey, and G. Prakash, "Top-gated graphene field-effect-transistors formed by decomposition of SiC," Applied Physics Letters, vol. 92, no. 9, p. 092102, 2008.

[68]Y. M. Lin, A. Valdes-Garcia, S. J. Han, D. B. Farmer, I. Meric, Y. Sun, Y. Wu, C. Dimitrakopoulos, A. Grill, and P. Avouris, "Wafer-scale graphene integrated circuit," Science, vol. 332, no. 6035, pp. 1294-1297, 2011.

[69]Z. Guo, R. Dong, P. S. Chakraborty, N. Lourenco, J. Palmer, Y. Hu, M. Ruan, J. Hankinson, J. Kunc, and J. D. Cressler, "Record maximum oscillation frequency in Cface epitaxial graphene transistors," Nano letters, vol. 13, no. 3, pp. 942-947, 2013.

[70]G. Fiori and G. Iannaccone, "Multiscale modeling for graphene-based nanoscale transistors," Proceedings of the IEEE, vol. 101, no. 7, pp. 1653-1669, 2013.

[71]F. Schwierz, "Graphene transistors," Nature nanotechnology, vol. 5, no. 7, pp. 487496, 2010.

[72]Y. Zhang, Z. Jiang, J. Small, M. Purewal, Y.-W. Tan, M. Fazlollahi, J. Chudow, J. Jaszczak, H. Stormer, and P. Kim, "Landau-level splitting in graphene in high magnetic fields," Physical review letters, vol. 96, no. 13, p. 136806, 2006.

[73]M. Katsnelson, K. Novoselov, and A. Geim, "Chiral tunnelling and the Klein paradox in graphene," Nature physics, vol. 2, no. 9, pp. 620-625, 2006.

[74]A. C. Neto, F. Guinea, N. M. Peres, K. S. Novoselov, and A. K. Geim, "The electronic properties of graphene," Reviews of modern physics, vol. 81, no. 1, p. 109, 2009.

[75]P. J. Freitas, "Connections between special relativity, charge conservation, and quantum mechanics," arXiv preprint quant-ph/9710051, 1997.

[76](6 July). The molecular orbital model. Available: http://virtuallaboratory.colorado.edu/CLUE-Chemistry/chapters/chapter3txt-3.html

[77]P. J. Freitas, "Connections between special relativity, charge conservation, and quantum mechanics," arXiv preprint quant-ph/9710051, 1997.

[78]Z. Zhu and J. E. Hoffman, "Condensed-matter physics: Catching relativistic electrons," Nature, vol. 513, no. 7518, pp. 319-320, 2014.

[79]W. Gao, L. B. Alemany, L. Ci, and P. M. Ajayan, "New insights into the structure and reduction of graphite oxide," Nature chemistry, vol. 1, no. 5, pp. 403-408, 2009. 
[80]S. Borisenko, Q. Gibson, D. Evtushinsky, V. Zabolotnyy, B. Büchner, and R. J. Cava, "Experimental realization of a three-dimensional Dirac semimetal," Physical review letters, vol. 113, no. 2, p. 027603, 2014.

[81]M. Neupane, S.-Y. Xu, R. Sankar, N. Alidoust, G. Bian, C. Liu, I. Belopolski, T.-R. Chang, H.-T. Jeng, and H. Lin, "Observation of a three-dimensional topological Dirac semimetal phase in high-mobility Cd3As2," Nature communications, vol. 5, 2014.

[82]Z. Liu, J. Jiang, B. Zhou, Z. Wang, Y. Zhang, H. Weng, D. Prabhakaran, S. Mo, H. Peng, and P. Dudin, "A stable three-dimensional topological Dirac semimetal Cd3As2," Nature materials, vol. 13, no. 7, pp. 677-681, 2014.

[83]S. Jeon, B. B. Zhou, A. Gyenis, B. E. Feldman, I. Kimchi, A. C. Potter, Q. D. Gibson, R. J. Cava, A. Vishwanath, and A. Yazdani, "Landau quantization and quasiparticle interference in the three-dimensional Dirac semimetal Cd3As2," Nature materials, vol. 13, no. 9, pp. 851-856, 2014.

[84]K. Subrahmanyam, L. Panchakarla, A. Govindaraj, and C. Rao, "Simple method of preparing graphene flakes by an arc-discharge method," The Journal of Physical Chemistry C, vol. 113, no. 11, pp. 4257-4259, 2009.

[85]S. Abdolhosseinzadeh, H. Asgharzadeh, and H. S. Kim, "Fast and fully-scalable synthesis of reduced graphene oxide," Scientific reports, vol. 5, p. 10160, 2015.

[86]Y. M. Lin, C. Dimitrakopoulos, K. A. Jenkins, D. B. Farmer, H. Y. Chiu, A. Grill, and P. Avouris, "100-GHz transistors from wafer-scale epitaxial graphene," Science, vol. 327, no. 5966, pp. 662-662, 2010.

[87]H. Xu, Z. Zhang, H. Xu, Z. Wang, S. Wang, and L. M. Peng, "Top-gated graphene field-effect transistors with high normalized transconductance and designable dirac point voltage," ACS nano, vol. 5, no. 6, pp. 5031-5037, 2011.

[88]D. H. Lee, J. Yi, J. M. Lee, S. J. Lee, Y. J. Doh, H. Y. Jeong, Z. Lee, U. Paik, J. A. Rogers, and W. I. Park, "Engineering electronic properties of graphene by coupling with Si-rich, two-dimensional islands," ACS nano, vol. 7, no. 1, pp. 301-307, 2012.

[89]J. Bai, R. Cheng, F. Xiu, L. Liao, M. Wang, A. Shailos, K. L. Wang, Y. Huang, and X. Duan, "Very large magnetoresistance in graphene nanoribbons," Nature nanotechnology, vol. 5, no. 9, pp. 655-659, 2010.

[90]Y.-W. Son, M. L. Cohen, and S. G. Louie, "Energy gaps in graphene nanoribbons," Physical review letters, vol. 97, no. 21, p. 216803, 2006.

[91]P. K. Vabbina, P. Nayyar, A. P. Nayak, A. M. Katzenmeyer, V. Logeeswaran, N. Pala, M. S. Islam, and A. Talin, "Synthesis of crystalline $\mathrm{ZnO}$ nanostructures on arbitrary 
substrates at ambient conditions," in SPIE NanoScience+ Engineering, 2011, pp. 81060H-81060H-9: International Society for Optics and Photonics.

[92]C. Al-Amin, P. K. Vabbina, M. Karabiyik, R. Sinha, C. Wang, and N. Pala, "Bandgap engineering of single layer graphene by randomly distributed nanoparticles," Journal of Materials Science: Materials in Electronics, vol. 27, no. 7, pp. 7454-7459, 2016.

[93]S. Mehdi Aghaei and I. Calizo, "Band gap tuning of armchair silicene nanoribbons using periodic hexagonal holes," Journal of Applied Physics, vol. 118, no. 10, p. 104304, 2015.

[94]S. M. Aghaei and I. Calizo, "Density functional theory study on energy band gap of armchair silicene nanoribbons with periodic nanoholes," MRS Advances, vol. 1, no. 22, pp. 1613-1618, 2016.

[95]M. Kim, N. S. Safron, E. Han, M. S. Arnold, and P. Gopalan, "Fabrication and characterization of large-area, semiconducting nanoperforated graphene materials," Nano letters, vol. 10, no. 4, pp. 1125-1131, 2010.

[96]R. Balog, B. Jørgensen, L. Nilsson, M. Andersen, E. Rienks, M. Bianchi, M. Fanetti, E. Lægsgaard, A. Baraldi, and S. Lizzit, "Bandgap opening in graphene induced by patterned hydrogen adsorption," Nature materials, vol. 9, no. 4, pp. 315-319, 2010.

[97]F. Yavari, C. Kritzinger, C. Gaire, L. Song, H. Gulapalli, T. Borca-Tasciuc, P. M. Ajayan, and N. Koratkar, "Tunable bandgap in graphene by the controlled adsorption of water molecules," small, vol. 6, no. 22, pp. 2535-2538, 2010.

[98]K. Takeda and K. Shiraishi, "Theoretical possibility of stage corrugation in Si and Ge analogs of graphite," Physical Review B, vol. 50, no. 20, p. 14916, 1994.

[99]Q. Pang, Y. Zhang, J. M. Zhang, V. Ji, and K. W. Xu, "Electronic and magnetic properties of pristine and chemically functionalized germanene nanoribbons," Nanoscale, vol. 3, no. 10, pp. 4330-4338, 2011.

[100] P. Liang, Y. Liu, S. Xing, H. Shu, and B. Tai, "Electronic and magnetic properties of germanene: Surface functionalization and strain effects," Solid State Communications, vol. 226, pp. 19-24, 2016.

[101] S. Jiang, S. Butler, E. Bianco, O. D. Restrepo, W. Windl, and J. E. Goldberger, "Improving the stability and optical properties of germanane via one-step covalent methyl-termination," Nature communications, vol. 5, 2014.

[102] L. Zhang, P. Bampoulis, A. Rudenko, Q. v. Yao, A. Van Houselt, B. Poelsema, M. Katsnelson, and H. Zandvliet, "Structural and electronic properties of germanene on MoS 2," Physical review letters, vol. 116, no. 25, p. 256804, 2016. 
[103] S. Z. Butler, S. M. Hollen, L. Cao, Y. Cui, J. A. Gupta, H. R. Gutiérrez, T. F. Heinz, S. S. Hong, J. Huang, and A. F. Ismach, "Progress, challenges, and opportunities in two-dimensional materials beyond graphene," ACS nano, vol. 7, no. 4, pp. 2898-2926, 2013.

[104] L. Chen, C. C. Liu, B. Feng, X. He, P. Cheng, Z. Ding, S. Meng, Y. Yao, and K. Wu, "Evidence for Dirac fermions in a honeycomb lattice based on silicon," Physical review letters, vol. 109, no. 5, p. 056804, 2012.

[105] J. Zhao, H. Liu, Z. Yu, R. Quhe, S. Zhou, Y. Wang, C. C. Liu, H. Zhong, N. Han, and J. Lu, "Rise of silicene: A competitive 2D material," Progress in Materials Science, vol. 83, pp. 24-151, 2016.

[106] X. T. An, Y. Y. Zhang, J. J. Liu, and S. S. Li, "Quantum spin Hall effect induced by electric field in silicene," Applied Physics Letters, vol. 102, no. 4, p. 043113, 2013.

[107] W. Hu, Z. Li, and J. Yang, "Structural, electronic, and optical properties of hybrid silicene and graphene nanocomposite," The Journal of chemical physics, vol. 139, no. 15, p. 154704, 2013.

[108] C. Hwang, D. A. Siegel, S. K. Mo, W. Regan, A. Ismach, Y. Zhang, A. Zettl, and A. Lanzara, "Fermi velocity engineering in graphene by substrate modification," arXiv preprint arXiv:1208.0567, 2012.

[109] P. Hohenberg and W. Kohn, "Inhomogeneous electron gas," Physical review, vol. 136, no. 3B, p. B864, 1964.

[110] W. Kohn and L. J. Sham, "Self-consistent equations including exchange and correlation effects," Physical review, vol. 140, no. 4A, p. A1133, 1965.

[111] M. Born and R. Oppenheimer, "Zur quantentheorie der molekeln," Annalen der Physik, vol. 389, no. 20, pp. 457-484, 1927.

[112] P. Schwerdtfeger, "The pseudopotential approximation in electronic structure theory," ChemPhysChem, vol. 12, no. 17, pp. 3143-3155, 2011.

[113] M. C. Payne, M. P. Teter, D. C. Allan, T. Arias, and J. Joannopoulos, "Iterative minimization techniques for ab initio total-energy calculations: molecular dynamics and conjugate gradients," Reviews of Modern Physics, vol. 64, no. 4, p. 1045, 1992.

[114] R. G. Parr, "Density functional theory of atoms and molecules," in Horizons of Quantum Chemistry: Springer, 1980, pp. 5-15. 
[115] J. P. Perdew, K. Schmidt, V. Van Doren, C. Van Alsenoy, and P. Geerlings, "Jacob's ladder of density functional approximations for the exchange-correlation energy," in AIP Conference Proceedings, 2001, vol. 577, no. 1, pp. 1-20: AIP.

[116] J. P. Perdew, A. Ruzsinszky, J. Tao, V. N. Staroverov, G. E. Scuseria, and G. I. Csonka, "Prescription for the design and selection of density functional approximations: More constraint satisfaction with fewer fits," The Journal of chemical physics, vol. 123, no. 6, p. 062201, 2005.

[117] J. P. Perdew and A. Zunger, "Self-interaction correction to density-functional approximations for many-electron systems," Physical Review B, vol. 23, no. 10, p. $5048,1981$.

[118] J. P. Perdew and Y. Wang, "Accurate and simple analytic representation of the electron-gas correlation energy," Physical Review B, vol. 45, no. 23, p. 13244, 1992.

[119] S. H. Vosko, L. Wilk, and M. Nusair, "Accurate spin-dependent electron liquid correlation energies for local spin density calculations: a critical analysis," Canadian Journal of physics, vol. 58, no. 8, pp. 1200-1211, 1980.

[120] J. P. Perdew, J. A. Chevary, S. H. Vosko, K. A. Jackson, M. R. Pederson, D. J. Singh, and C. Fiolhais, "Atoms, molecules, solids, and surfaces: Applications of the generalized gradient approximation for exchange and correlation," Physical Review B, vol. 46, no. 11, p. 6671, 1992.

[121] J. P. Perdew, K. Burke, and M. Ernzerhof, "Generalized gradient approximation made simple," Physical review letters, vol. 77, no. 18, p. 3865, 1996.

[122] A. D. Becke, "Density-functional exchange-energy approximation with correct asymptotic behavior," Physical review A, vol. 38, no. 6, p. 3098, 1988.

[123] C. Lee, W. Yang, and R. G. Parr, "Development of the Colle-Salvetti correlationenergy formula into a functional of the electron density," Physical review B, vol. 37, no. 2 , p. $785,1988$.

[124] J. Tao, J. P. Perdew, V. N. Staroverov, and G. E. Scuseria, "Climbing the density functional ladder: Nonempirical meta-generalized gradient approximation designed for molecules and solids," Physical Review Letters, vol. 91, no. 14, p. 146401, 2003.

[125] A. D. Becke, "Density-functional thermochemistry. III. The role of exact exchange," The Journal of chemical physics, vol. 98, no. 7, pp. 5648-5652, 1993.

[126] P. Stephens, F. Devlin, C. Chabalowski, and M. J. Frisch, "Ab initio calculation of vibrational absorption and circular dichroism spectra using density functional force fields," Journal of Physical Chemistry, vol. 98, no. 45, pp. 11623-7, 1994. 
[127] S. Grimme, "Semiempirical GGA-type density functional constructed with a longrange dispersion correction," Journal of computational chemistry, vol. 27, no. 15, pp. 1787-1799, 2006.

[128] S. Grimme, J. Antony, S. Ehrlich, and H. Krieg, "A consistent and accurate ab initio parametrization of density functional dispersion correction (DFT-D) for the 94 elements H-Pu," The Journal of chemical physics, vol. 132, no. 15, p. 154104, 2010.

[129] S. Datta, Electronic transport in mesoscopic systems. Cambridge university press, 1997.

[130] M. Brandbyge, J. L. Mozos, P. Ordejón, J. Taylor, and K. Stokbro, "Densityfunctional method for nonequilibrium electron transport," Physical Review B, vol. 65, no. 16, p. 165401, 2002.

[131] K. Stokbro, J. Taylor, M. Brandbyge, and H. Guo, "Ab-initio non-equilibrium Green's function formalism for calculating electron transport in molecular devices," in Introducing Molecular Electronics: Springer, 2006, pp. 117-151.

[132] H. Haug, A. P. Jauho, and M. Cardona, Quantum kinetics in transport and optics of semiconductors. Springer, 2008.

[133] M. Kan, J. Zhou, Y. Li, and Q. Sun, "Using carbon chains to mediate magnetic coupling in zigzag graphene nanoribbons," Applied Physics Letters, vol. 100, no. 17, p. 173106, 2012.

[134] M. Kan, J. Zhou, Q. Sun, Q. Wang, Y. Kawazoe, and P. Jena, "Tuning magnetic properties of graphene nanoribbons with topological line defects: From antiferromagnetic to ferromagnetic," Physical Review B, vol. 85, no. 15, p. 155450, 2012.

[135] T. Wassmann, A. P. Seitsonen, A. M. Saitta, M. Lazzeri, and F. Mauri, "Structure, stability, edge states, and aromaticity of graphene ribbons," Physical Review Letters, vol. 101, no. 9, p. 096402, 2008.

[136] G. Lee and K. Cho, "Electronic structures of zigzag graphene nanoribbons with edge hydrogenation and oxidation," Physical Review B, vol. 79, no. 16, p. 165440, 2009.

[137] K. A. Ritter and J. W. Lyding, "The influence of edge structure on the electronic properties of graphene quantum dots and nanoribbons," Nature materials, vol. 8, no. 3, pp. 235-242, 2009.

[138] S. Bhandary, O. Eriksson, B. Sanyal, and M. I. Katsnelson, "Complex edge effects in zigzag graphene nanoribbons due to hydrogen loading," Physical Review B, vol. 82, no. 16, p. 165405, 2010. 
[139] J. Kunstmann, C. Özdoğan, A. Quandt, and H. Fehske, "Stability of edge states and edge magnetism in graphene nanoribbons," Physical Review B, vol. 83, no. 4, p. $045414,2011$.

[140] P. Wagner, C. P. Ewels, V. V. Ivanovskaya, P. R. Briddon, A. Pateau, and B. Humbert, "Ripple edge engineering of graphene nanoribbons," Physical Review B, vol. 84, no. 13, p. 134110, 2011.

[141] X. Zheng, X. Wang, L. Huang, H. Hao, J. Lan, and Z. Zeng, "Stabilizing the ground state in zigzag-edged graphene nanoribbons by dihydrogenation," Physical Review B, vol. 86, no. 8, p. 081408, 2012.

[142] C. Si, J. Liu, Y. Xu, J. Wu, B. L. Gu, and W. Duan, "Functionalized germanene as a prototype of large-gap two-dimensional topological insulators," Physical Review B, vol. 89, no. 11, p. 115429, 2014.

[143] Z. Ni, Q. Liu, K. Tang, J. Zheng, J. Zhou, R. Qin, Z. Gao, D. Yu, and J. Lu, "Tunable bandgap in silicene and germanene," Nano letters, vol. 12, no. 1, pp. 113-118, 2011.

[144] B. van den Broek, M. Houssa, E. Scalise, G. Pourtois, V. Afanas'ev, and A. Stesmans, "First-principles electronic functionalization of silicene and germanene by adatom chemisorption," Applied Surface Science, vol. 291, pp. 104-108, 2014.

[145] W. Xia, W. Hu, Z. Li, and J. Yang, "A first-principles study of gas adsorption on germanene," Physical Chemistry Chemical Physics, vol. 16, no. 41, pp. 22495-22498, 2014.

[146] D. Wang, W. Gu, Y. Zhang, Y. Hu, T. Zhang, X. Tao, and W. Chen, "Novel C-rich carbon nitride for room temperature $\mathrm{NO}_{2}$ gas sensors," RSC Advances, vol. 4, no. 35 , pp. 18003-18006, 2014.

[147] S. M. Aghaei and I. Calizo, "Bandgap changes in armchair silicene nanoribbons perforated with periodic nanoholes," in SoutheastCon 2015, 2015, pp. 1-6: IEEE.

[148] A. ToolKit, "QuantumWise A/S," S, www. quantum wise. com, 2014.

[149] J. M. Soler, E. Artacho, J. D. Gale, A. García, J. Junquera, P. Ordejón, and D. Sánchez-Portal, "The SIESTA method for ab initio order-N materials simulation," Journal of Physics: Condensed Matter, vol. 14, no. 11, p. 2745, 2002.

[150] S. Aghaei, M. Monshi, and I. Calizo, "A theoretical study of gas adsorption on silicene nanoribbons and its application in a highly sensitive molecule sensor," $R S C$ Advances, vol. 6, no. 97, pp. 94417-94428, 2016. 
[151] J. Prasongkit, R. G. Amorim, S. Chakraborty, R. Ahuja, R. H. Scheicher, and V. Amornkitbamrung, "Highly Sensitive and Selective Gas Detection Based on Silicene," The Journal of Physical Chemistry C, vol. 119, no. 29, pp. 16934-16940, 2015.

[152] P. Wagner, V. V. Ivanovskaya, M. Melle-Franco, B. Humbert, J. J. Adjizian, P. R. Briddon, and C. P. Ewels, "Stable hydrogenated graphene edge types: normal and reconstructed Klein edges," Physical Review B, vol. 88, no. 9, p. 094106, 2013.

[153] K. Reuter and M. Scheffler, "Composition, structure, and stability of RuO 2 (110) as a function of oxygen pressure," Physical Review B, vol. 65, no. 3, p. 035406, 2001.

[154] G. Soldano, M. Juarez, B. Teo, and E. Santos, "Structure and stability of graphene edges in $\mathrm{O} 2$ and $\mathrm{H} 2$ environments from ab initio thermodynamics," Carbon, vol. 78, pp. 181-189, 2014.

[155] D. R. Stull and H. Prophet, "JANAF thermochemical tables," DTIC Document1971.

[156] M. Winter, "WebElements," 1999.

[157] G. Guzmán-Verri and L. L. Y. Voon, "Band structure of hydrogenated Si nanosheets and nanotubes," Journal of Physics: Condensed Matter, vol. 23, no. 14, p. 145502, 2011.

[158] F. B. Zheng, C. W. Zhang, P. J. Wang, and S. S. Li, "Novel half-metal and spin gapless semiconductor properties in N-doped silicene nanoribbons," Journal of Applied Physics, vol. 113, no. 15, p. 154302, 2013.

[159] J. M. Zhang, W. T. Song, K. W. Xu, and V. Ji, "The study of the P doped silicene nanoribbons with first-principles," Computational Materials Science, vol. 95, pp. 429434, 2014.

[160] H. X. Luan, C. W. Zhang, F.-B. Zheng, and P. J. Wang, "First-principles study of the electronic properties of $\mathrm{B} / \mathrm{N}$ atom doped silicene nanoribbons," The Journal of Physical Chemistry C, vol. 117, no. 26, pp. 13620-13626, 2013.

[161] F. B. Zheng, C. W. Zhang, S. S. Yan, and F. Li, "Novel electronic and magnetic properties in N or B doped silicene nanoribbons," Journal of Materials Chemistry C, vol. 1, no. 15, pp. 2735-2743, 2013.

[162] L. Ma, J. M. Zhang, K. W. Xu, and V. Ji, "Structural and electronic properties of substitutionally doped armchair silicene nanoribbons," Physica B: Condensed Matter, vol. 425, pp. 66-71, 2013. 
[163] E. Bianco, S. Butler, S. Jiang, O. Restrepo, W. Windl, and J. Goldberger, "Stability and exfoliation of germanane: a germanium graphane analogue," ACS nano, vol. 7, no. 5, p. 4414, 2013.

[164] N. J. Roome and J. D. Carey, "Beyond graphene: stable elemental monolayers of silicene and germanene," ACS Appl Mater Interfaces, vol. 6, no. 10, pp. 7743-7750, 2014.

[165] M. M. Monshi, S. M. Aghaei, and I. Calizo, "Band Gap Opening and Optical Absorption Enhancement in Graphene using ZnO Nanoclusters," arXiv preprint arXiv:1706.06163, 2017.

[166] S. M. Aghaei and I. Calizo, "Band gap tuning of armchair silicene nanoribbons using periodic hexagonal holes," Journal of Applied Physics, vol. 118, no. 10, p. 104304, 2015.

[167] T. Hussain, T. Kaewmaraya, S. Chakraborty, and R. Ahuja, "Defect and SubstitutionInduced Silicene Sensor to Probe Toxic Gases," The Journal of Physical Chemistry C, vol. 120, no. 44, pp. 25256-25262, 2016.

[168] S. M. Aghaei, I. Torres, and I. Calizo, "Structural Stability of Functionalized Silicene Nanoribbons with Normal, Reconstructed, and Hybrid Edges," Journal of Nanomaterials, vol. 2016, p. 8, 2016, Art. no. 5959162.

[169] Y. He, P. Zhang, X. Hou, J. Xu, M. Wang, Y. Wu, J. Qu, and M. Dong, "Adjusting the electronic properties of silicon carbide nanoribbons by introducing edge functionalization," RSC Advances, vol. 4, no. 66, pp. 35042-35047, 2014.

[170] M. M. Monshi, S. M. Aghaei, and I. Calizo, "Edge functionalized germanene nanoribbons: impact on electronic and magnetic properties," RSC Advances, vol. 7, no. 31, pp. 18900-18908, 2017.

[171] A. N. Abbas, G. Liu, B. Liu, L. Zhang, H. Liu, D. Ohlberg, W. Wu, and C. Zhou, "Patterning, characterization, and chemical sensing applications of graphene nanoribbon arrays down to $5 \mathrm{~nm}$ using helium ion beam lithography," ACS nano, vol. 8, no. 2, pp. 1538-1546, 2014.

[172] A. N. Abbas, G. Liu, A. Narita, M. Orosco, X. Feng, K. Müllen, and C. Zhou, "Deposition, characterization, and thin-film-based chemical sensing of ultra-long chemically synthesized graphene nanoribbons," Journal of the American Chemical Society, vol. 136, no. 21, pp. 7555-7558, 2014.

[173] B. Huang, Z. Li, Z. Liu, G. Zhou, S. Hao, J. Wu, B. L. Gu, and W. Duan, "Adsorption of gas molecules on graphene nanoribbons and its implication for nanoscale molecule 
sensor," The Journal of Physical Chemistry C, vol. 112, no. 35, pp. 13442-13446, 2008.

[174] A. Saffarzadeh, "Modeling of gas adsorption on graphene nanoribbons," Journal of Applied Physics, vol. 107, no. 11, p. 114309, 2010.

[175] H. J. Yoon, J. H. Yang, Z. Zhou, S. S. Yang, and M. M. C. Cheng, "Carbon dioxide gas sensor using a graphene sheet," Sensors and Actuators B: Chemical, vol. 157, no. 1, pp. 310-313, 2011.

[176] F. Schedin, A. Geim, S. Morozov, E. Hill, P. Blake, M. Katsnelson, and K. Novoselov, "Detection of individual gas molecules adsorbed on graphene," Nature materials, vol. 6, no. 9, pp. 652-655, 2007.

[177] M. Ye, R. Quhe, J. Zheng, Z. Ni, Y. Wang, Y. Yuan, G. Tse, J. Shi, Z. Gao, and J. Lu, "Tunable band gap in germanene by surface adsorption," Physica E: Lowdimensional Systems and Nanostructures, vol. 59, pp. 60-65, 2014.

[178] F. Costanzo, P. L. Silvestrelli, and F. Ancilotto, "Physisorption, diffusion, and chemisorption pathways of $\mathrm{H} 2$ molecule on graphene and on $(2,2)$ carbon nanotube by first principles calculations," Journal of chemical theory and computation, vol. 8, no. 4, pp. 1288-1294, 2012.

[179] S. Aghaei, M. Monshi, I. Torres, and I. Calizo, "Efficient and Reversible CO2 Capture by Lithium-functionalized Germanene Monolayer," arXiv preprint arXiv:1705.05801, 2017.

[180] S. K. Gupta, D. Singh, K. Rajput, and Y. Sonvane, "Germanene: a new electronic gas sensing material," RSC Advances, vol. 6, no. 104, pp. 102264-102271, 2016.

[181] J. E. Padilha and R. B. Pontes, "Electronic and transport properties of structural defects in monolayer germanene: An ab initio investigation," Solid State Communications, vol. 225, pp. 38-43, 2016.

[182] J. Taylor, H. Guo, and J. Wang, "Ab initio modeling of quantum transport properties of molecular electronic devices," Physical Review B, vol. 63, no. 24, p. 245407, 2001.

[183] Atomistix ToolKit (ATK) QuantumWise Simulator [Online]. Available: http://www.quantumwise.com

[184] S. Grimme, C. Mück-Lichtenfeld, and J. Antony, "Noncovalent interactions between graphene sheets and in multishell (hyper) fullerenes," The Journal of Physical Chemistry C, vol. 111, no. 30, pp. 11199-11207, 2007. 
[185] H. J. Monkhorst and J. D. Pack, "Special points for Brillouin-zone integrations," Physical review B, vol. 13, no. 12, p. 5188, 1976.

[186] J. Setiadi, M. D. Arnold, and M. J. Ford, "Li-ion adsorption and diffusion on twodimensional silicon with defects: a first principles study," ACS applied materials \& interfaces, vol. 5, no. 21, pp. 10690-10695, 2013.

[187] X. Q. Wang, H. D. Li, and J. T. Wang, "Induced ferromagnetism in one-side semihydrogenated silicene and germanene," Physical Chemistry Chemical Physics, vol. 14, no. 9, pp. 3031-3036, 2012.

[188] W. H. Tao and C. H. Tsai, " $\mathrm{H}_{2} \mathrm{~S}$ sensing properties of noble metal doped $\mathrm{WO}_{3}$ thin film sensor fabricated by micromachining," Sensors and Actuators B: Chemical, vol. 81, no. 2, pp. 237-247, 2002.

[189] S. M. Aghaei, M. M. Monshi, and I. Calizo, "A theoretical study of gas adsorption on silicene nanoribbons and its application in a highly sensitive molecule sensor," RSC Advances, vol. 6, no. 97, pp. 94417-94428, 2016.

[190] Q. Zhang, Y. Bai, Z. Yang, J. Tian, and Z. Meng, "Effect of sulfur dioxide inhalation on the expression of K ATP and L-Ca 2+ channels in rat hearts," Environmental toxicology and pharmacology, vol. 39, no. 3, pp. 1132-1138, 2015.

[191] K. J. Lee and S. J. Kim, "Theoretical investigation of $\mathrm{CO}_{2}$ adsorption on graphene," Bull. Korean Chem. Soc, vol. 34, no. 10, pp. 3022-3026, 2013.

[192] J. Sivek, H. Sahin, B. Partoens, and F. M. Peeters, "Adsorption and absorption of boron, nitrogen, aluminum, and phosphorus on silicene: Stability and electronic and phonon properties," Physical Review B, vol. 87, no. 8, p. 085444, 2013.

[193] C. J. Rupp, S. Chakraborty, R. Ahuja, and R. J. Baierle, "The effect of impurities in ultra-thin hydrogenated silicene and germanene: a first principles study," Physical Chemistry Chemical Physics, vol. 17, no. 34, pp. 22210-22216, 2015.

[194] R. S. Mulliken, "Electronic population analysis on LCAO-MO molecular wave functions. I," The Journal of Chemical Physics, vol. 23, no. 10, pp. 1833-1840, 1955.

[195] V. O. Özçelik, H. H. Gurel, and S. Ciraci, "Self-healing of vacancy defects in singlelayer graphene and silicene," Physical Review B, vol. 88, no. 4, p. 045440, 2013.

[196] X. Liang, Y.-S. Jung, S. Wu, A. Ismach, D. L. Olynick, S. Cabrini, and J. Bokor, "Formation of bandgap and subbands in graphene nanomeshes with sub- $10 \mathrm{~nm}$ ribbon width fabricated via nanoimprint lithography," Nano letters, vol. 10, no. 7, pp. 2454$2460,2010$. 
[197] N. Gao, J. Li, and Q. Jiang, "Bandgap opening in silicene: Effect of substrates," Chemical Physics Letters, vol. 592, pp. 222-226, 2014.

[198] V. Surya, K. Iyakutti, H. Mizuseki, and Y. Kawazoe, "Tuning electronic structure of graphene: a first-principles study," IEEE Transactions on Nanotechnology, vol. 11, no. 3, pp. 534-541, 2012.

[199] M. Monshi, S. Aghaei, and I. Calizo, "Doping and Defect-Induced Germanene: A Superior Media for Sensing $\mathrm{H}_{2} \mathrm{~S}, \mathrm{SO}_{2}$, and $\mathrm{CO}_{2}$ gas molecules," arXiv preprint arXiv:1707.03508, 2017.

[200] Z. H. Ni, T. Yu, Y. H. Lu, Y. Y. Wang, Y. P. Feng, and Z. X. Shen, "Uniaxial strain on graphene: Raman spectroscopy study and band-gap opening," ACS nano, vol. 2, no. 11, pp. 2301-2305, 2008.

[201] K. Lee, S. Park, and J. Lee, "Rapid Ag recovery using photocatalytic ZnO nanopowders prepared by solution-combustion method," Journal of Materials Science Letters, vol. 22, no. 1, pp. 65-67, 2003.

[202] T. Xu, L. Zhang, H. Cheng, and Y. Zhu, "Significantly enhanced photocatalytic performance of $\mathrm{ZnO}$ via graphene hybridization and the mechanism study," Applied Catalysis B: Environmental, vol. 101, no. 3, pp. 382-387, 2011.

[203] J. M. Matxain, J. M. Mercero, J. E. Fowler, and J. M. Ugalde, "Electronic excitation energies of Zn i O i clusters," Journal of the American Chemical Society, vol. 125, no. 31, pp. 9494-9499, 2003.

[204] Y. Meir and N. S. Wingreen, "Landauer formula for the current through an interacting electron region," Physical review letters, vol. 68, no. 16, p. 2512, 1992.

[205] W. A. Harrison, Solid state theory. Courier Corporation, 1980.

[206] D. J. Griffiths, "Introduction to electrodynamics," ed: AAPT, 2005.

[207] S. S. Carara, R. J. Batista, and H. Chacham, "Modifications in graphene electron states due to a deposited lattice of Au nanoparticles: Density functional calculations," Physical Review B, vol. 80, no. 11, p. 115435, 2009.

[208] C. Stampfer, J. Güttinger, S. Hellmüller, F. Molitor, K. Ensslin, and T. Ihn, "Energy gaps in etched graphene nanoribbons," Physical review letters, vol. 102, no. 5, p. 056403, 2009.

[209] T. Mueller, F. Xia, and P. Avouris, "Graphene photodetectors for high-speed optical communications," Nature Photonics, vol. 4, no. 5, pp. 297-301, 2010. 
[210] S. Rathi, I. Lee, D. Lim, J. Wang, Y. Ochiai, N. Aoki, K. Watanabe, T. Taniguchi, G.-H. Lee, and Y. J. Yu, "Tunable electrical and optical characteristics in monolayer graphene and few-layer MoS2 heterostructure devices," Nano letters, vol. 15, no. 8 , pp. 5017-5024, 2015.

[211] W. Zhang, C. P. Chuu, J. K. Huang, C. H. Chen, M. L. Tsai, Y. H. Chang, C. T. Liang, Y. Z. Chen, Y. L. Chueh, and J. H. He, "Ultrahigh-gain photodetectors based on atomically thin graphene-MoS2 heterostructures," Scientific reports, vol. 4, p. 3826, 2014.

[212] R. Paschotta, "Photodetectors," RP Photonics Consulting GmbH.[Online]. Available: http://www. rp-photonics. com, 2010.

[213] K. F. Mak, C. Lee, J. Hone, J. Shan, and T. F. Heinz, "Atomically thin $\mathrm{MoS}_{2}$ : a new direct-gap semiconductor," Physical Review Letters, vol. 105, no. 13, p. 136805, 2010.

[214] W. Choi, M. Y. Cho, A. Konar, J. H. Lee, G. B. Cha, S. C. Hong, S. Kim, J. Kim, D. Jena, and J. Joo, "High-detectivity multilayer $\mathrm{MoS}_{2}$ phototransistors with spectral response from ultraviolet to infrared," Advanced Materials, vol. 24, no. 43, pp. 58325836, 2012.

[215] D. S. Tsai, K. K. Liu, D. H. Lien, M. L. Tsai, C. F. Kang, C. A. Lin, L. J. Li, and J. H. He, "Few-layer MoS2 with high broadband photogain and fast optical switching for use in harsh environments," Acs Nano, vol. 7, no. 5, pp. 3905-3911, 2013.

[216] O. Lopez-Sanchez, D. Lembke, M. Kayci, A. Radenovic, and A. Kis, "Ultrasensitive photodetectors based on monolayer $\mathrm{MoS}_{2}$," Nature nanotechnology, vol. 8, no. 7, pp. 497-501, 2013.

[217] H. Wang, T. Taychatanapat, A. Hsu, K. Watanabe, T. Taniguchi, P. Jarillo-Herrero, and T. Palacios, "BN/graphene/BN transistors for RF applications," IEEE Electron Device Letters, vol. 32, no. 9, pp. 1209-1211, 2011.

[218] D. Jena, "Tunneling transistors based on graphene and 2-D crystals," Proceedings of the IEEE, vol. 101, no. 7, pp. 1585-1602, 2013.

[219] J. Yang, D. Voiry, S. J. Ahn, D. Kang, A. Y. Kim, M. Chhowalla, and H. S. Shin, "Two-Dimensional Hybrid Nanosheets of Tungsten Disulfide and Reduced Graphene Oxide as Catalysts for Enhanced Hydrogen Evolution," Angewandte Chemie International Edition, vol. 52, no. 51, pp. 13751-13754, 2013.

[220] T. Georgiou, R. Jalil, B. D. Belle, L. Britnell, R. V. Gorbachev, S. V. Morozov, Y.J. Kim, A. Gholinia, S. J. Haigh, and O. Makarovsky, "Vertical field-effect transistor based on graphene- $\mathrm{WS}_{2}$ heterostructures for flexible and transparent electronics," Nature nanotechnology, vol. 8, no. 2, pp. 100-103, 2013. 
[221] K. Roy, M. Padmanabhan, S. Goswami, T. P. Sai, G. Ramalingam, S. Raghavan, and A. Ghosh, "Graphene-MoS 2 hybrid structures for multifunctional photoresponsive memory devices," Nature nanotechnology, vol. 8, no. 11, pp. 826-830, 2013.

[222] S. Bertolazzi, D. Krasnozhon, and A. Kis, "Nonvolatile memory cells based on MoS2/graphene heterostructures," ACS nano, vol. 7, no. 4, pp. 3246-3252, 2013.

[223] S. Ryu, L. Liu, S. Berciaud, Y. J. Yu, H. Liu, P. Kim, G. W. Flynn, and L. E. Brus, "Atmospheric oxygen binding and hole doping in deformed graphene on a $\mathrm{SiO}_{2}$ substrate," Nano letters, vol. 10, no. 12, pp. 4944-4951, 2010.

[224] M. Ghorbani-Asl, P. D. Bristowe, K. Koziol, T. Heine, and A. Kuc, "Effect of compression on the electronic, optical and transport properties of $\mathrm{MoS}_{2} /$ graphenebased junctions," 2D Materials, vol. 3, no. 2, p. 025018, 2016.

[225] M. Radecka, M. Rekas, A. Trenczek-Zajac, and K. Zakrzewska, "Importance of the band gap energy and flat band potential for application of modified $\mathrm{TiO}_{2}$ photoanodes in water photolysis," Journal of power sources, vol. 181, no. 1, pp. 46-55, 2008. 
VITA

\section{MONIROJJAMAN MONSHI}

$2000-2004$

B. Sc., Applied Physics, Electronics and Communication Engineering

University of Dhaka

Dhaka, Bangladesh

$2009-2012$

M. Sc., Applied Physics, Electronics and Communication

Engineering

University of Dhaka

Dhaka, Bangladesh

$2012-2017$

Doctoral Candidate $(\mathrm{PhD})$

Florida International University

Miami, Florida, USA

Publications and Presentations

Journal Papers:

[1] M. M. Monshi, S. M. Aghaei, and I. Calizo, "Edge functionalized germanene nanoribbons: impact on electronic and magnetic properties," RSC Advances, vol. 7, no. 31, pp. 18900-18908, 2017.

[2] M. M. Monshi, S. M. Aghaei, and I. Calizo, "Band Gap Opening and Optical Absorption Enhancement in Graphene using ZnO Nanoclusters," Physica E: Lowdimensional Systems and Nanostructures, under review (minor revision and preparing the response letter to the reviewer).

[3] M. M. Monshi, S. M. Aghaei, and I. Calizo, " Doping and Defect-Induced Germanene: A Superior Media for Sensing $\mathrm{H}_{2} \mathrm{~S}, \mathrm{SO}_{2}$, and $\mathrm{CO}_{2}$ gas molecules", Surface Science, under review. 
[4] S. M. Aghaei, M. M. Monshi, I. Torres, and I. Calizo, "Edge functionalization and doping effects on the stability, electronic and magnetic properties of silicene nanoribbons," RSC Advances, vol. 6, no. 21, pp. 17046-17058, 2016.

[5] S. M. Aghaei, M. M. Monshi, and I. Calizo, "A theoretical study of gas adsorption on silicene nanoribbons and its application in a highly sensitive molecule sensor," RSC Advances, vol. 6, no. 97, pp. 94417-94428, 2016.

[1] M. M. Monshi, S. M. Aghaei and I. Calizo, "Functionalized Bandgap Tuning of Germanene," Functionalized Bandgap tuning of Germanene" - Oral Presentation at MRS Fall Meeting and Exhibit, 2015, Boston, Massachusetts, US, 2015.

[2] M. M. Monshi, S. M. Aghaei and I. Calizo, "Edge Functionalized Band Gap Tuning of Germanene", Oral Presentation at Workshop on the "Science and technology of 2D materials" at the University of Central Florida, Orlando, Florida, US, 2015. 